\title{
On rigidity and the isomorphism problem for tree braid groups
}

\author{
Lucas Sabalka
}

\begin{abstract}
We solve the isomorphism problem for braid groups on trees with $n=4$ or 5 strands. We do so in three main steps, each of which is interesting in its own right. First, we establish some tools and terminology for dealing with computations using the cohomology of tree braid groups, couching our discussion in the language of differential forms. Second, we show that, given a tree braid group $B_{n} T$ on $n=4$ or 5 strands, $H^{*}\left(B_{n} T\right)$ is an exterior face algebra. Finally, we prove that one may reconstruct the tree $T$ from a tree braid group $B_{n} T$ for $n=4$ or 5. Among other corollaries, this third step shows that, when $n=4$ or 5 , tree braid groups $B_{n} T$ and trees $T$ (up to homeomorphism) are in bijective correspondence. That such a bijection exists is not true for higher dimensional spaces, and is an artifact of the 1-dimensionality of trees. We end by stating the results for right-angled Artin groups corresponding to the main theorems, some of which do not yet appear in the literature.
\end{abstract}

Mathematics Subject Classification (2000). Primary 20F65, 20F36; Secondary 55N99, 13D25, 20F10.

Keywords. Tree braid groups, configuration spaces, isomorphism problem, exterior face algebras, discrete Morse theory, group cohomology, right-angled Artin groups.

\section{Introduction}

Given a graph $\Gamma$, the unlabelled configuration space $U \mathcal{C}^{n} \Gamma$ of $n$ points on $\Gamma$ is the space of $n$-element subsets of distinct points in $\Gamma$. The $n$-strand braid group of $\Gamma$, denoted $B_{n} \Gamma$, is the fundamental group of $U \mathcal{C}^{n} \Gamma$. If $\Gamma$ is a tree, $B_{n} \Gamma$ is a tree braid group.

Graph braid groups are of interest because of their connections with classical braid groups (see, for instance, [23]) and right-angled Artin groups [8], [24], [16], as well as connections in robotics and mechanical engineering. Graph braid groups can, for instance, model the motions of robots moving about a factory floor [17], [11], [12], or the motions of microscopic balls of liquid on a nano-scale electronic circuit [18].

Ghrist [17] showed that the complexes $U \mathcal{C}^{n} \Gamma$ are $K\left(B_{n} \Gamma, 1\right)$ spaces. Abrams [1] showed that graph braid groups are fundamental groups of locally CAT(0) cubical complexes, and so for instance have solvable word and conjugacy problem [3]. Crisp and Wiest [8] showed that any graph braid group embeds in some right-angled Artin 
group, so graph braid groups are linear, bi-orderable, and residually finite. For more information on what is known about graph braid groups, see for instance [23].

For any class of groups $\mathcal{E}$, it is interesting to ask whether or not one can algorithmically decide if two members $G$ and $G^{\prime}$ in $\mathcal{E}$ are isomorphic as groups. We call this question the isomorphism problem for $\mathscr{E}$.

Isomorphism problems are one of the fundamental topics of study for combinatorial and geometric group theory. Isomorphism problems are the hardest of the three classes of algorithmic problems in group theory formulated by Max Dehn [9]. It is known that the isomorphism problem for finitely presented groups is undecidable in general [2], [22]. However, there are solutions to the isomorphism problem for certain classes of groups. A short list of such classes of groups includes: polycyclic-by-finite groups [25], finitely generated nilpotent groups [19], torsion-free word hyperbolic groups which do not split over the trivial or infinite cyclic group [26], and finitely generated fully residually free groups [4].

The purpose of this paper is to implement an algorithm to solve the isomorphism problem for tree braid groups in some cases. We prove:

Theorem A (The isomorphism problem, cf. Theorem 7.1). Let $G$ and $G^{\prime}$ be two groups be given by finite presentations, and assume that $G \cong B_{n} T$ and $G^{\prime} \cong B_{n} T^{\prime}$ for some positive integer $n$ and finite trees $T$ and $T^{\prime}$. If either:

- $n=4$ or 5 or

- at least one of $G$ or $G^{\prime}$ is free,

then there exists an algorithm which decides whether $G$ and $G^{\prime}$ are isomorphic. The trees $T$ and $T^{\prime}$ need not be specified. If one of $T$ and $T^{\prime}$ has at least 3 essential vertices, then $n$ need not be specified.

To prove Theorem A, the main ingredient is a bijection between trees and tree braid groups. This bijection allows us to algorithmically reconstruct the defining tree $T$ from the tree braid group $B_{n} T$. This reduces the isomorphism problem for tree braid groups to the isomorphism problem for trees, which has a brute force algorithmic solution.

The bijection between trees and tree braid groups is the strongest and most difficult result of this paper, and is interesting in its own right:

Theorem B (Rigidity for 4 and 5 strand tree braid groups, cf. Theorem 6.9). Let $T$ and $T^{\prime}$ be two finite trees, and let $n=4$ or 5 . The tree braid groups $B_{n} T$ and $B_{n} T^{\prime}$ are isomorphic as groups if and only if the trees $T$ and $T^{\prime}$ are homeomorphic as trees.

The idea of the proof of Theorem B is to use cohomology to reconstruct the tree $T$ from the tree braid group $B_{n} T$. This reconstruction involves careful combinatorial bookkeeping in a finite simplicial complex $\Delta$ associated to $B_{n} T$. The complex $\Delta$ is the defining complex for an exterior face algebra structure on the cohomology ring 
of $B_{n} T$ (see Section 2.2 for definitions). The main technical used to prove Theorem $\mathrm{B}$ is that $\Delta$ exists and is unique:

Theorem $\mathbf{C}$ (Exterior face algebra structure on cohomology, cf. Theorem 5.2). Let $T$ be a finite tree. For $n=4$ or $5, H^{*}\left(B_{n} T ; \mathbb{Z} / 2 \mathbb{Z}\right)$ is an exterior face algebra. The simplicial complex $\Delta$ defining the exterior face algebra structure is unique and at most 1-dimensional.

Theorem $\mathrm{C}$ and other related results lead to an almost complete characterization of when the cohomology of a tree braid group is an exterior face algebra (see Conjecture 5.17).

Throughout the proofs of Theorems B and C, we rely extensively on results due to discrete Morse theory, many of which were presented in previous papers: [15], [13], [16], [14]. We also develop tools to discuss many properties of cohomology rings of tree braid groups, using the language of differential forms.

The remainder of this paper is organized as follows. In Section 2, we introduce terminology about trees that we will need to prove the main results, and define exterior face algebras. In Section 3, we survey results from other sources needed in our proofs. We pay particular attention to the structure of the cohomology ring for tree braid groups, as detailed in [16]. In Section 4, we develop the notation and terminology to talk about the cohomology rings of graph braid groups in the language of differential forms. In Sections 5 and 6, we prove a number of results which lead to Theorems 5.2 and 6.9, respectively. The solution to the isomorphism problem for tree braid groups on 4 or 5 strands then follows in Section 7. Finally, in Section 8, we end with theorems for right-angled Artin groups in a similar vein to Theorems A, B, and C.

The author would like to thank the following people for their help in writing this paper: his postdoctoral advisor, Misha Kapovich; his doctoral advisor, Ilya Kapovich; Daniel Farley, for numerous helpful discussions on this matter; and Go Fujita for initially proposing this problem.

\section{Terminology}

2.1. Trees. We begin with terminology for trees. Throughout this subsection, see Figure 1 for explicit examples of some of the many of the concepts we define.

Let $T$ be a tree. We call a vertex $v$ of $T$ essential if $v$ has degree 3 or more. Two essential vertices are considered adjacent if they are connected by a path which crosses no other essential vertices. An essential vertex $v$ is extremal it is adjacent to exactly one other essential vertex. A tree is linear if there exists an embedded line segment which contains every essential vertex; equivalently, if it has at most two extremal vertices. 


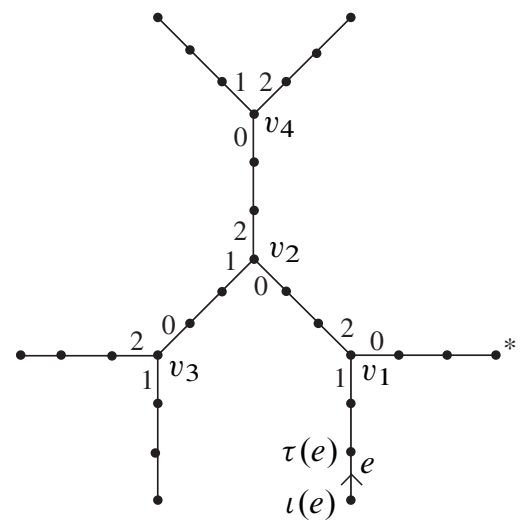

Figure 1. This figure shows an embedding of a tree in the plane. The tree is $T_{\min }$, the minimal nonlinear tree - i.e. the (unique up to homeomorphism) nonlinear tree with the fewest number of essential vertices and the smallest degrees of essential vertices. With the choice of basepoint $*$, we have a Morse $T_{\min }$-embedding. The essential vertices of $T_{\min }$ are labelled $v_{1}, v_{2}, v_{3}$, and $v_{4}$. The vertices $v_{1}, v_{3}$, and $v_{4}$ are extremal, and each are adjacent only to $v_{2}$. Directions from each essential vertex are labelled. For instance, $v_{2}$ is in direction 2 from $v_{1}$, and $v_{4}$ is in direction 0 from $v_{3}$. The edge $e$ has its endpoints $\iota(e)$ and $\tau(e)$ labelled. Here, $T_{\min }$ is sufficiently subdivided for $n=4$.

Definition 2.1 (Morse $T$-embedding). Let $T$ be a tree. Embed $T$ into the plane. Let * denote a degree 1 vertex of $T$, called the basepoint of $T$. The information of the tree $T$, the embedding of $T$ into the plane, and the choice of $*$ is called a Morse T-embedding.

Let $T$ be a tree with a Morse $T$-embedding. Let $e$ be an edge in $T$. We call the endpoint of $e$ closer to $*$ in $T$ the terminal vertex of $e$, denoted $\tau(e)$. This convention gives us an orientation on edges in $T$. Similarly, the endpoint of $e$ further from $*$ in $T$ is the initial vertex of $e$, denoted $\iota(e)$. This convention gives us an orientation on edges in $T$.

Let $S$ be a collection of vertices and edges of $T$. We denote by $T-S$ the largest closed subgraph of $T$ which does not contain an element of $S$. In other words, $T-S$ is formed by deleting every edge in $S$, as well as any vertex in $S$ and any edge with an endpoint in $S$.

Let $v$ be a vertex in $T$. Given a Morse $T$-embedding, the edges adjacent to $v$ may be numbered $0, \ldots, \operatorname{deg}(v)-1$ in the order encountered by a clockwise traversal of $T$ from $*$, and where $\operatorname{deg}(v)$ denotes the degree of $v$. If $v=*$, the unique edge adjacent to $*$ is numbered 1. A direction from $v$ is a choice of one of these edge labels. A vertex is said to lie in direction $d$ from $v$ if $d$ labels the first edge of the unique simple path (that is, a path with no self intersections) from $v$ to the vertex. By convention, $v$ lies in direction 0 from itself. An edge lies in direction $d$ from $v$ if, for 
one of the endpoints of $e, d$ labels the first edge of the unique simple path from $v$ to that endpoint.

An extremal vertex has the property that every other essential vertex lies in a single direction from it.

Let $\Delta^{\prime}$ denote the union of those open cells of $\prod^{n} \Gamma$ whose closures intersect the fat diagonal $\Delta=\left\{\left(x_{1}, \ldots, x_{n}\right) \mid x_{i}=x_{j}\right.$ for some $\left.i \neq j\right\}$. Let $U \mathscr{D}^{n} \Gamma$ denote the quotient of the space $\prod^{n} \Gamma-\Delta^{\prime}$ by the action of the symmetric group given by permuting coordinates. Note that $U \mathscr{D}^{n} \Gamma$ inherits a $\mathrm{CW}$ complex structure from the Cartesian product: an open cell in $U \mathscr{D}^{n} \Gamma$ has the form $\left\{y_{1}, \ldots, y_{n}\right\}$ such that each $y_{i}$ is either a vertex or an edge and the closures of the $y_{i}$ are mutually disjoint. The set notation is used to indicate that order does not matter. We call $U \mathcal{D}^{n} \Gamma$ the unlabelled discretized configuration space of $\Gamma$. Under most circumstances, the $U \mathcal{C}^{n} \Gamma$ is homotopy equivalent to $U \mathscr{D}^{n} \Gamma$. Specifically:

Theorem 2.2 (Sufficient subdivision; [21] for $n=2$, [1] for $n>2$ ). For any $n>1$ and any graph $\Gamma$ with at least $n$ vertices, $U \mathcal{C}^{n} \Gamma$ strong deformation retracts onto $U \mathscr{D}^{n} \Gamma$ if

(1) each path between distinct vertices of degree not equal to 2 passes through at least $n-1$ edges; and

(2) each path from a vertex to itself which is not null-homotopic in $\Gamma$ passes through at least $n+1$ edges.

A graph $\Gamma$ satisfying the conditions of this theorem for a given $n$ is called sufficiently subdivided for $n$. It is clear that, for any $n$, every graph is homeomorphic to a sufficiently subdivided graph for $n$.

Throughout the rest of this paper, we assume that we are dealing with graphs which are sufficiently subdivided for at least $n+2$ strands.

We mention here that Abrams [1] proved that the universal cover of the space $U \mathscr{D}^{n} \Gamma$ is a CAT(0) cubical complex for any graph $\Gamma$. This implies that graph braid groups have solvable word and conjugacy problems [3].

2.2. Exterior face algebras. We now consider exterior face algebras. Let $K$ be a finite simplicial complex with vertices $\left\{v_{1}, \ldots, v_{k}\right\}$. For any field $F$ with identity, the exterior face algebra $\Lambda_{F}(K)$ of $K$ over $F$ is the quotient of the exterior algebra $\Lambda_{F}\left[v_{1}, \ldots, v_{k+1}\right]$ by the ideal generated by products of vertices of non-faces of $K$. In other words, $\Lambda_{F}(K)$ is the $F$-vector space having the products $v_{i_{1}} v_{i_{2}} \ldots v_{i_{j}}$ $\left(0 \leq j \leq k, i_{1}<i_{2}<\cdots<i_{j}\right)$ as a basis, and subject to the following multiplicative relations:

- $v_{i} v_{j}=-v_{j} v_{i}$ for $0 \leq i, j \leq n$,

- $v_{i}^{2}=0$ for $0 \leq i \leq n$, and

- $v_{i_{1}} \ldots v_{i_{k}}=0$ if $i_{1}<\cdots<i_{k}$ and $\left\{v_{i_{1}}, \ldots, v_{i_{k}}\right\}$ is not a face of $K$. 
An exterior algebra corresponds to the exterior face algebra of a standard simplex. Note the shift of indices: an $i$-cell in $K$ corresponds to an element of degree $i+1$ in $\Lambda(K)$.

For our purposes, $F$ will always be the field $\mathbb{Z} / 2 \mathbb{Z}$, so we suppress the subscript $F$ from now on. For this field, $\Lambda(K)$ is a quotient of a polynomial ring:

$$
\Lambda(K)=(\mathbb{Z} / 2 \mathbb{Z})\left[v_{1}, \ldots, v_{n}\right] / I(K),
$$

where $I(K)$ is the ideal of $(\mathbb{Z} / 2 \mathbb{Z})\left[v_{1}, \ldots, v_{n}\right]$ generated by the set

$$
\left\{v_{1}^{2}, \ldots, v_{n}^{2}\right\} \cup\left\{v_{i_{1}} \ldots v_{i_{k}} \mid i_{1}<\cdots<i_{k} ;\left\{v_{i_{1}}, \ldots, v_{i_{k}}\right\} \text { is not a face of } K\right\} .
$$

In the $\mathbb{Z} / 2 \mathbb{Z}$ case, Gubeladze [20] has shown that exterior face algebras are in bijective correspondence with their defining simplicial complexes:

Theorem 2.3 (Gubeladze's theorem on exterior face algebra rigidity; [20]). Let $K$ and $K^{\prime}$ be two finite simplicial complexes. Then $\Lambda(K)$ and $\Lambda\left(K^{\prime}\right)$ are isomorphic as algebras if and only if $K$ and $K^{\prime}$ are isomorphic as simplicial complexes.

This rigidity allows us to speak of 'the' simplicial complex defining an exterior face algebra.

As an example application of Gubeladze's theorem, see Theorem 8.3 in Section 8, on right-angled Artin groups.

\section{Previous results}

Now that we have introduced notation and terminology concerning trees and exterior face algebras, we turn our attention to recalling previous results - in particular important results from [15], [13], and [16]. We begin with the first two references, on fundamental group and homology.

3.1. Morse theory, the fundamental group, and homology. For a tree $T$, consider a Morse $T$-embedding (Definition 2.1). By [15], this embedding induces a 'Morse matching' on $U D^{n} T$. For the sake of brevity, we do not define or detail the Morse matching here, but instead define the cells of $U D^{n} T$ which are critical with respect to this matching. More detailed expositions on the Morse matching and the classification of cells of $U D^{n} T$ can be found in [23], where a Morse matching is referred to as a discrete gradient vector field (the exposition in [23] is based on the original work in [15]).

Definition 3.1 (Blocked, respectful, and critical). Begin with a Morse $T$-embedding. Let $c$ be an open cell in $U D^{n} T$. Consider a vertex $v \in c$. If $v=*$, then $v$ is blocked by $*$ in $c$. If $v \neq *$, let $e$ be the unique edge in $T$ with $\iota(e)=v$. If $e \cap x \neq \emptyset$ for 
some edge or vertex $x \in c, x \neq v$, then again $v$ is blocked by $x$ in $c$. If $v$ is not blocked in $c, v$ is unblocked.

Now consider an edge $e \in c$. The edge $e$ is disrespectful in $c$ if: there exists a vertex $v \in c$ blocked by $e$ and which, in a clockwise traversal of the tree $T$ from $*$, is traversed after one endpoint of $e$ but before the other. Otherwise, the edge $e$ is respectful in $c$.

A cell $c$ is critical if there are neither unblocked vertices nor respectful edges in $c$. See Figure 2 for an example.

Critical cells were seen to be very useful in describing graph braid groups, as evidenced in [15], [16], [13], etc. In particular, as we will see, critical cells lend themselves well to describing generating sets and even relations for fundamental group, homology, and cohomology.

A $k$-cell in $U \mathscr{D}^{n} T$ corresponds exactly to $n-k$ strands sitting on $T$ and $k$ strands simultaneously crossing $k$ disjoint edges. To see the connection between critical cells and braids, consider a critical 1-cell $c=\left\{v_{1}, \ldots, v_{n-1}, e\right\}$ with unique edge $e \in c$. Then $c$ corresponds to the following braid: start with $n$ strands sitting next to $*$ in $T$. Move each strand out to a distinct vertex in $\left\{v_{1}, \ldots, v_{n-1}, \iota(e)\right\}$ in order so that the strand furthest away from $*$ goes to the highest-numbered vertex, the second-furthest goes to the second-highest, etc. Next, move the strand at $\iota(e)$ to $\tau(e)$. Finally, move each of the strands back to $*$, in order so that the strand on the lowest-numbered vertex in $\left\{v_{1}, \ldots, v_{n-1}, \tau(e)\right\}$ moves first and ends nearest $*$, the second-lowest ends second-nearest, etc. See Figure 2.

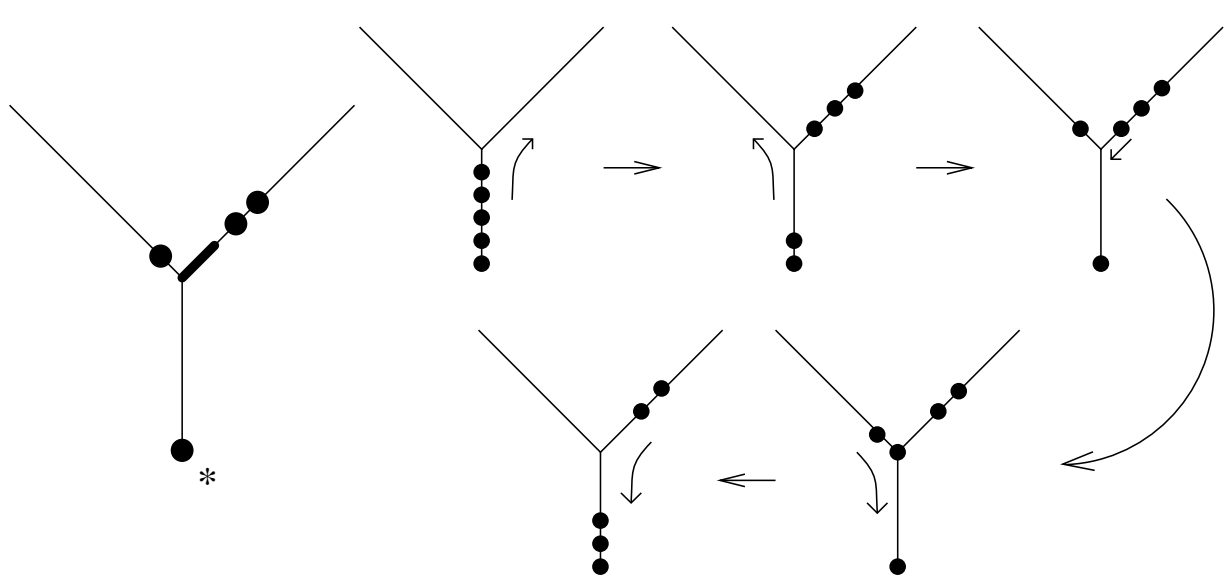

Figure 2. On the left is a critical 1-cell $c$. The remaining figures are a diagrammatic illustration of the braid that $c$ represents.

The power of discrete Morse theory and the classification of critical cells is evident in the following useful theorems: 
Theorem 3.2 (Morse presentation for $B_{n} T$; [15], corollary of Theorem 2.5). Let $T$ be a tree. Fix a Morse T-embedding. Then $B_{n} T$ has a presentation for which the generators may be identified with the set of critical 1-cells, and the relations are determined by the set of critical 2-cells.

A presentation derived from a Morse $T$-embedding as in Theorem 3.2 is called a Morse presentation, and its generators are Morse generators.

Theorem 3.3 (Homology; [13]). Let $T$ be a tree. Fix a Morse T-embedding. Then $H_{i} B_{n} T$ is free abelian of rank equal to the cardinality of the set of critical $i$-cells of $T$. In particular, $H_{i} B_{n} T$ has a generating set which may be identified with the set of critical $i$-cells of $T$.

Such a distinguished basis for homology is called a Morse basis, composed of elements called Morse generators.

We end this subsection with the following useful calculation about certain tree braid groups, to which we will repeatedly refer:

Theorem 3.4 (Radial rank; [15], Corollary 4.2). If $\Gamma$ is a radial tree -i.e. a tree with exactly one essential vertex $v$-then the tree braid group $B_{n} \Gamma$ is free of rank

$$
Y_{n}(x):=\sum_{i=2}^{x-1}\left[\left(\begin{array}{c}
n+x-2 \\
n-1
\end{array}\right)-\left(\begin{array}{c}
n+x-i-1 \\
n-1
\end{array}\right)\right],
$$

where $x=\operatorname{deg}(v)$.

Note that the function $Y_{n}(x)$ is a monotonically increasing function of $x$ for $x \geq 3$ and of $n$ for $n \geq 2$. For instance, $Y_{2}(x)=x^{2} / 2-3 x / 2+1$, and $Y_{3}(x)=$ $x^{3} / 3-x^{2} / 2-5 x / 6+1$.

3.2. Cohomology. To describe the structure of cohomology for tree braid groups, we first need to introduce some definitions. We begin by defining a partial ordering and an equivalence relation on cells of $U D^{n} T$. Using this partial order and equivalence relation, we are able to state two of the main results from [16], which are computational and structural statements about cohomology for tree braid groups, and will be referred to often. We end the section with some notation, suggested by one of these results, for what will be called reduced 1-cells.

Let $E(c)$ denote the set of edges of the $i$-cell $c$. We abuse the notation by also letting $E(c)$ denote the subset $\bigcup_{e \in E(c)} e$ of $T$. For two cells $c$ and $c^{\prime}$, write $c \sim c^{\prime}$ if

(1) $E(c)=E\left(c^{\prime}\right)$, and

(2) for any connected component $C$ of $T-E(c)$,

$$
|C \cap(c-E(c))|=\left|C \cap\left(c^{\prime}-E\left(c^{\prime}\right)\right)\right| .
$$


It is straightforward to see that $\sim$ is an equivalence relation on the set of open cells in $U \mathscr{D}^{n} T$. Let $[c]$ denote the equivalence class of a cell $c$.

If every vertex in $c$ is blocked, we say $c$ is reduced. If $c$ is reduced but there exists an edge of $c$ such that $\tau(e)$ is not essential, $c$ is extraneous. If $c$ is reduced, $c$ is not a 0 -cell, and for every edge $e$ of $c$ no vertex of $c$ is blocked by $\tau(e)$, then again $c$ is extraneous.

We define a partial order $\leq$ on the equivalence classes based on the face relation $\leq$ on cells, writing $\left[c_{0}\right] \leq\left[c_{1}\right]$ if there exist representatives $\hat{c}_{0} \in\left[c_{0}\right], \hat{c}_{1} \in\left[c_{1}\right]$ such that $\hat{c}_{0} \leq \hat{c}_{1}$-i.e. $\hat{c}_{0}$ is a face of $\hat{c}_{1}$. We record some properties of $\sim$ and $\leq$ here:

Theorem 3.5 (Properties of $\leq$ and $\sim$; c.f. [16]).

(1) The relation $\leq$ is indeed a partial order.

(2) Suppose that $c_{1}, \ldots, c_{k}$ are 1-cells from distinct equivalence classes. If the set $\left\{\left[c_{1}\right], \ldots,\left[c_{k}\right]\right\}$ has an upper bound $[s]$ with respect to $\leq$, then the collection has a least upper bound. Furthermore, if $e_{1}, \ldots, e_{k}$ are the edges of $T$ satisfying $e_{i} \in c_{i}$, then the edges $e_{1}, \ldots, e_{k}$ are the edges of $s$ and are pairwise disjoint.

(3) For any $k$-cell $s$, there exists a unique collection $\left\{\left[c_{1}\right], \ldots,\left[c_{k}\right]\right\}$ of equivalence classes of 1-cells such that $[s]$ is the least upper bound of $\left\{\left[c_{1}\right], \ldots,\left[c_{k}\right]\right\}$ with respect to $\leq$.

(4) If $c$ is a critical cell in $U \mathscr{D}^{n} \Gamma$ and $\left[c^{\prime}\right] \leq[c]$, then $c^{\prime} \sim \hat{c}$ for some critical cell $\hat{c}$.

(5) There exists exactly one reduced cell in each -equivalence class. Every critical cell is reduced, but not every reduced cell is critical. In particular, a critical cell is the unique critical cell in its equivalence class.

Proof. Most of this theorem is from [16], Lemma 4.1 and the preceding discussion. The only part not proven in [16] is Part (5).

For Part (5), that every equivalence class contains a reduced cell is clear. That every critical cell is reduced follows from the definitions. That there exist reduced cells which are not critical is clear. That a critical cell is the unique critical cell in its equivalence class follows from the first two statements of Part (5), and was also proven in [16].

It remains to prove that two reduced cells $c_{1}$ and $c_{2}$ in the same equivalence class must be equal. By [16], Lemma 4.1(1), $E\left(c_{1}\right)=E\left(c_{2}\right)$, so let $E:=E\left(c_{1}\right)=E\left(c_{2}\right)$. Consider a connected component $C$ of $T-E$. Let $*_{C}$ be the smallest vertex of $C$. The idea of the proof is that $T$ is sufficiently subdivided so that any collection of at most $n-1$ blocked vertices inside of $C$ must be 'stacked up' at $*_{C}$, and is in particular uniquely determined. The uniqueness will force $c_{1}$ and $c_{2}$ to coincide, for each such connected component. We formalize this idea.

Since $*_{C}$ is the smallest vertex of $C$, either $*_{C}=*$ or $e\left(*_{C}\right)$ intersects an edge in $E$. Define a vertex $v_{C}$ of $T$ as follows. If $*_{C}=*$, let $v_{C}:=*$. If $*_{C} \neq *$, then since $*_{C}$ is the smallest vertex of $C$, there exists an edge $e \in E$ with $e\left(*_{C}\right) \cap e \neq \emptyset$. 
In this case, define $*_{C}$ to be $\tau(e)$. In the former case, $v_{C}$ has degree 1 in $T$, and in the latter case, $v_{C}$ has degree at least 3 in $T$. Since $T$ is sufficiently subdivided for $n+2$ strands, $v_{C}$ is at least $n+1$ edges away from any other vertex of $T$ which does not have degree 2 in $T$. Since $*_{C}$ is at most 2 edges away from $v_{C}, *_{C}$ is at least $n-1$ edges away from any other vertex of $T$ which does not have degree 2 in $T$. Since each edge of $E$ has an essential terminal endpoint, $*_{C}$ is at least $n-2$ edges away from any other vertex of $C$ which does not have degree 2 in $C$. Thus, since $C$ is a (connected subset of a) tree, for any $i=0, \ldots, n-1$, there is a unique vertex $v_{C, i}$ in $C$ such that the unique path from $v_{C, i}$ to $*_{C}$ contains exactly $k$ edges.

By the definition of $\sim, c_{1}$ and $c_{2}$ have the same number $k$ of vertices in $C$. We claim that both $c_{1}$ and $c_{2}$ contain the $k$ vertices $v_{C, 1}, \ldots, v_{C, k}$ of $C$. For, assume otherwise. Without loss of generality, assume $c_{1}$ does not contain the vertex $v_{C, i}$. Let $v$ be the smallest vertex of $c_{1} \cap C$ greater than $v_{C, i}$. Then $\tau(e(v))$ is in $C$, since $*_{C}$ is in the same direction from $v_{C, i}$ in $T$ as $*$ is. But $\tau(e(v))$ does not intersect $c_{1}$, since $C$ contains only vertices of $c_{1}$ and $v$ was chosen to be as small as possible. Thus, $v$ is unblocked in $c_{1}$. This contradicts the hypothesis that $c_{1}$ is reduced. Thus, $c_{1}$ and $c_{2}$ have the same edge set $E$ and the same vertices in each connected component of $T-E, c_{1}=c_{2}$.

Let $C_{*}\left(U D^{n} T\right)$ denote the cellular chain complex of chains of cells in $U D^{n} T$ with coefficients in $\mathbb{Z} / 2 \mathbb{Z}$. Let $\phi_{[c]}: C_{*}\left(U D^{n} T\right) \rightarrow \mathbb{Z} / 2 \mathbb{Z}$ denote the characteristic function of the $\sim$-equivalence class of $c: \phi_{[c]}\left(c^{\prime}\right)=1$ if and only if $c^{\prime} \sim c$. For $c$ a critical cell, let $c^{*}: H_{*}\left(B_{n} T\right) \rightarrow \mathbb{Z} / 2 \mathbb{Z}$ denote the dual of $c$ viewed as a basis element of cohomology, by the Universal Coefficient Theorem. Note the boundary maps for chains are all 0 [13], so $H^{*}\left(B_{n} T\right) \cong \operatorname{Hom}\left(H_{*}\left(B_{n}, T\right), \mathbb{Z} / 2 \mathbb{Z}\right)$. Then, using the ordering $\leq$ on equivalence classes of 1-cells, we may state the following theorem:

Theorem 3.6 (Cohomology; [16], Proposition 4.5 and preceding discussion). Let $T$ be a tree. Fix a Morse T-embedding (see Definition 2.1). Then under the induced Morse matching the following holds:

(1) If $c$ is a critical cell in $U D^{n} T$, then $c^{*}=\left[\phi_{[c]}\right]$. A distinguished basis for $i$-dimensional cohomology is

$$
\left\{c^{*} \mid \text { c a critical } i \text {-cell }\right\} \text {. }
$$

(2) Let $s$ be a critical $i$-cell in $U D^{n} T$. Let $[s]$ be the least upper bound of $\left\{\left[c_{1}\right], \ldots,\left[c_{i}\right]\right\}$, where the $\left[c_{1}\right], \ldots,\left[c_{i}\right]$ are distinct equivalence classes of 1-cells. Then, without loss of generality, $c_{1}, \ldots, c_{i}$ are critical, and

$$
c_{1}^{*} \cup \cdots \cup c_{i}^{*}=s^{*} .
$$

In particular, $H^{*}\left(B_{n} T\right)$ is generated as a ring by duals of critical 1-cells. 
(3) If $\left[c_{1}\right], \ldots,\left[c_{i}\right]$ are distinct equivalence classes of critical 1 -cells having the least upper bound $[s]$, then

$$
\left[\phi_{\left[c_{1}\right]}\right] \cup \cdots \cup\left[\phi_{\left[c_{i}\right]}\right]=\left[\phi_{[s]}\right] .
$$

If $\left[c_{1}\right], \ldots,\left[c_{i}\right]$ are not all pairwise distinct or have no upper bound, then $\left[\phi_{\left[c_{1}\right]}\right] \cup \cdots \cup\left[\phi_{\left[c_{i}\right]}\right]=0$.

Note a critical cell has dimension at most $\left(\left\lfloor\frac{n}{2}\right\rfloor\right)$ [15]. It follows from the first part of Theorem 3.6 that cohomology is trivial in all dimensions greater than $\left\lfloor\frac{n}{2}\right\rfloor$.

As with homology, such a distinguished basis for cohomology is called a Morse basis, composed of elements called Morse generators. The power of this theorem comes from the characterization not only of a Morse basis, but of the cup product structure.

3.3. Reduced cells. Throughout the remainder of this paper, we will be extensively using reduced cells and computations involving reduced cells. For future reference, we establish here notation for many reduced 1-cells, and record some properties of reduced cells.

For a vector $\vec{x} \in \mathbb{N}^{\infty}$ of nonnegative integers, indexed from 0 to $\infty$, we let $x_{i}$ denote the $i^{\text {th }}$ entry of $\vec{x}$. Given a tree $T$, a Morse $T$-embedding, and an essential vertex $v$ of $T$, the vector $\vec{x}$ is a $v$-vector if $x_{i}=0$ for all $i \geq \operatorname{deg}(v)$. When writing $v$-vectors, we will omit the entries 0 for indices $i \geq \operatorname{deg}(v)$. The length of $\vec{x}$, denoted $|\vec{x}|$, is the sum $\sum_{i=0}^{\infty} x_{i}$ (all of our lengths will be finite).

Let $c$ be a reduced 1-cell of $U D^{n} T$ with unique edge $e$ such that $e$ has an essential terminal endpoint $a=\tau(e)$. By Theorem 3.5, $c$ is unique in its equivalence class $[c]$. Thus, $c$ is uniquely determined by the number of vertices in each connected component of $T-e$. This means that $c$ is uniquely determined by specifying the endpoint $a=\tau(e)$, the direction $d$ from $a$ along $e$, and the number of vertices in $c$ in each direction from $a$. Let $\vec{x} \in \mathbb{N}^{\infty}$ be the $a$-vector such that, for $i \in$ $\{0, \ldots, \operatorname{deg}(a)-1\} x_{i}$ is the number of vertices in $c$ in direction $i$ from $a$. Note $n=|\vec{x}|$.

Definition 3.7 (Reduced 1-cell notation). To encode the reduced 1-cell $c$ with edge $e$ such that $\tau(e)$ is the essential vertex $a$, we write

$$
(a, d, \vec{x}) \text { or equivalently }(a, e, \vec{x}),
$$

where $d$ and $\vec{x}$ are as above. We say that $\vec{x}$ is the a-vector for $c$, and that $c$ lies over the vertex $a$.

This notation is a slightly modified version of the notation developed in the paper [15], and appears in [16].

Using this notation, a fairly immediate observation is: 
Lemma 3.8. Let $c=(a, e, \vec{x})$ be a non-extraneous reduced 1 -cell. Then $x_{0} \leq n-2$.

Proof. Let $d$ be the direction from $a$ to $\iota(e)$. Since $e$ is not extraneous, there exists some vertex $v$ of $c$ such that the direction $d^{\prime}$ from $a$ to $v$ is not 0 or $d$. Thus, $x_{d}, x_{d^{\prime}} \geq 1$. But $|\vec{x}|=n$, so $x_{0} \leq n-2$.

As Theorem 3.6 suggests, upper bounds of equivalence classes of reduced cells play an important role. We record many properties of upper bounds in the following lemma. Note that, in the proof of the last statement, we explicitly construct the reduced representative of upper bound of the equivalence classes of two non-extraneous reduced 1-cells.

Lemma 3.9 (Upper Bound Lemma). Let $c_{1}=(a, d, \vec{x})$ and $c_{2}=(b, f, \vec{y})$ be nonextraneous reduced 1 -cells where $a \leq b$ in the order on vertices. Let $\alpha$ be the direction from a to $b$. Then:

(1) the direction from $b$ to $a$ is 0.

(2) $\left\{\left[c_{1}\right],\left[c_{2}\right]\right\}$ has an upper bound if and only if

(a) $a \neq b$, and

(b) $x_{\alpha}+y_{0} \geq n+\epsilon$, where $\epsilon=\epsilon\left(c_{1}, \alpha\right)$ is 1 if $d=\alpha$ and 0 otherwise.

(1) If $\left\{\left[c_{1}\right],\left[c_{2}\right]\right\}$ has an upper bound, then $x_{\alpha} \geq 2+\epsilon$.

(2) If $\left\{\left[c_{1}\right],\left[c_{2}\right]\right\}$ has an upper bound $[s]$, let $s$ be the reduced representative of $[s]$. We may explicitly describe s. The edge $f$ of $c_{2}$ is disrespectful in the reduced representative $s$ of $[s]$ if and only if $f$ is disrespectful in $c_{2}$. The edge e of $c_{1}$ is disrespectful in $s$ if and only if either

(a) $0<\alpha<d$ and $x_{\alpha}+y_{0}>n$, or

(b) there exists some $i \neq \alpha$ such that $0<i<d$ and $x_{i}>0$.

The $\epsilon\left(c_{1}, \alpha\right)$ in the lemma is called the upper bound constant in direction $\alpha$ for $c_{1}$.

Proof. (1) If $a=b$, then the direction from $b=a$ to itself is by definition 0 . If $a<b$, that the direction from $b$ to $a$ is 0 follows directly from the definition of $a<b$.

(2) If $\left\{\left[c_{1}\right],\left[c_{2}\right]\right\}$ has an upper bound, let $[s]$ be a least upper bound. By Theorem 3.5, $[s]$ exists, is unique, and has a unique element - say, $s$ - in which all vertices are blocked. Since $\tau(e)$ and $\tau(f)$ are essential, $s$ is reduced. Also by Theorem 3.5, the edges $e$ and $f$ are disjoint - in particular, $a \neq b$. Finally, note that $x_{\alpha}$ is the number of elements of not just $c_{1}$ but also $s$ in direction $\alpha$ from $a$, and similarly $y_{\beta}$ is the number of elements of $s$ in direction $\beta$ from $b$. Since $\beta=0, n-y_{\beta}$ is the number of elements of $s$ in all directions not equal to $\beta$ from $b$. Since $T$ is a tree, $n-y_{\beta}$ must be at most the number of elements in $s$ in direction $\alpha$ from $a-$ that is, $x_{\alpha}$. But if $d=\alpha$, then the edge of $c_{1}$ which is also an edge of $s$ is in direction $\alpha$ from $a$ but direction $\beta$ from $b$. Thus if $d=\alpha, n-y_{\beta}$ must be at most $x_{\alpha}-1$. The desired inequality then follows. 
Now assume that $c_{1}$ and $c_{2}$ satisfy the desired properties. Let $c_{1}^{\prime}$ be the 1-cell with $\left(n-x_{\alpha}+\epsilon\right)$ strands on $T$ consisting of the edge of $c_{1}$ and all vertices of $c_{1}$ not in direction $\alpha$ from $a$. Similarly, let $c_{2}^{\prime}$ be the 1-cell with $\left(n-y_{\beta}\right)$ strands on $T$ consisting of the edge of $c_{2}$ and all vertices of $c_{2}$ not in direction $\alpha$ from $a$. Since $a \neq b$, we may define the 2-cell $s^{\prime}$ to be $c_{1}^{\prime} \cup c_{2}^{\prime}$ with $k$ strands on $T$, where $k=\left(n-x_{\alpha}+\epsilon\right)+\left(n-y_{\beta}\right)$. Since $x_{\alpha}+y_{\beta} \geq n+\epsilon, k \leq n$. Since $T$ is sufficiently subdivided for $n+2$, there are at least $n$ vertices of $T$ between $a$ and $b$ not contained in $s^{\prime}$. Let $s$ be the 2-cell with $n$ strands on $T$ which is $s^{\prime}$ plus exactly $n-k$ vertices between $a$ and $b$. Then by the definitions of $\sim$ and $\leq,[s]$ is an upper bound for $\left\{\left[c_{1}\right],\left[c_{2}\right]\right\}$.

(3) If $\left\{\left[c_{1}\right],\left[c_{2}\right]\right\}$ has an upper bound, then $x_{\alpha} \geq n+\epsilon-y_{0} \geq 2+\epsilon$, as $y_{0} \leq n-2$ by Lemma 3.8 .

(4) If $\left\{\left[c_{1}\right],\left[c_{2}\right]\right\}$ has an upper bound $[s]$, then by Theorem 3.5, the reduced representative $s$ of $[s]$ is unique. We explicitly construct $s$.

Let $s_{1}$ be the reduced cell on $y_{0}$ strands which is $c_{1}$ but with only $x_{\alpha}-\left(n-y_{0}\right)$ strands in direction $\alpha$ from $a$. As $x_{\alpha} \geq n+\epsilon-y_{0}$, even if $e$ is in direction $\alpha$ from $a, s_{1}$ still contains the edge $e$. The cell $s_{1}$ corresponds to deleting the $n-y_{0}$ largest strands with respect to the order on vertices in the direction $\alpha$ from $a$. Let $s_{2}$ be the reduced cell on $n-y_{0}$ strands which is $c_{2}$ but with the vertices in direction 0 from $b$ removed. Finally, let $s=s_{1} \cup s_{2}$. Since each of $s_{1}$ and $s_{2}$ is reduced, so is $\alpha$. Note $f$ is disrespectful in $c_{2}$ if and only if $f$ is disrespectful in $s_{2}$, if and only if $f$ is disrespectful in $s$, by the definition of disrespectful. Since $e$ is disrespectful in $c_{1}$, $e$ will remain disrespectful in $s_{1}$ and therefore $s$ unless there was only one direction $i$ such that $0<i<d$ and $x_{i}>0$ - namely, $i=\alpha$ - and there are no vertices in direction $i=\alpha$ from $a$ in $s_{1}$. There are no vertices in direction $\alpha$ from $a$ in $s_{1}$ if and only if $0<\alpha<d$ and $x_{\alpha}+y_{0}=n$. This finishes the proof of the lemma.

\section{Cohomology in terms of differential forms}

We will couch our further discussion of cohomology of tree braid groups in the terminology of de Rham cohomology and differential forms. Although we are dealing with CW complexes instead of manifolds, hopefully the similarity of the formulas, particularly in defining differentials, will justify our abuse of notation.

Recall that $C_{*}\left(U D^{n} T\right)$ is the cellular chain complex on $U D^{n} T$. We denote certain cochains on our space by the term form, where a $k$-form will be a $k$-cochain. The 0 -forms will be functions on cellular chains in $C_{*}\left(U D^{n} T\right)$ which take values in $\mathbb{Z} / 2 \mathbb{Z}$.

For a vertex $v \in T$ and a direction $i$ from $v$, let $D_{v, i}$ be the function from cells of $U D^{n} T$ to $\mathbb{Z}$ which takes a cell $c$ and counts the number of vertices or edges in $c$ in direction $i$ from $v$. Thus, for instance, for a reduced cell $(a, d, \vec{x}), D_{a, i}(a, d, \vec{x})=x_{i}$. Let $\bar{D}_{v, i}$ be similarly a function which takes a cell $c$ and counts the number of vertices or edges in $c$ in direction $i$ from $v$, but where each edge of $v$ is considered 
in the direction of its terminal endpoint from $v$ instead of its initial endpoint. Thus, for instance, $\bar{D}_{a, i}(a, d, \vec{x})=x_{i}$ if $i \neq 0, d$, but $\bar{D}_{a, 0}(a, d, \vec{x})=x_{0}+1$ and $\bar{D}_{a, d}(a, d, \vec{x})=x_{d}-1$.

Example 4.1. Consider the 2-cell $s$ shown in Figure 3. This figure depicts a 2-cell $s$ on a tree with three essential vertices $a, b$, and $c$. We have:

$D_{a, i}(s)=\left\{\begin{array}{ll}0 & \text { if } i=0, \\ 6 & \text { if } i=1, \\ 1 & \text { if } i=2,\end{array} \quad D_{b, i}(s)=\left\{\begin{array}{ll}1 & \text { if } i=0, \\ 1 & \text { if } i=1, \\ 3 & \text { if } i=2, \\ 2 & \text { if } i=3,\end{array}, \quad \bar{D}_{b, i}(s)= \begin{cases}2 & \text { if } i=0, \\ 1 & \text { if } i=1, \\ 3 & \text { if } i=2, \\ 1 & \text { if } i=3\end{cases}\right.\right.$

In terms of differential forms, the following is an incomplete list of forms which map $c$ to 1 :

$$
\begin{gathered}
f\left(a,\left[\begin{array}{l}
0 \\
6 \\
1
\end{array}\right]\right), \quad f\left(b,\left[\begin{array}{l}
2 \\
1 \\
3 \\
1
\end{array}\right]\right), \quad f\left(c,\left[\begin{array}{l}
6 \\
1 \\
0
\end{array}\right]\right), \\
f\left(a,\left[\begin{array}{l}
0 \\
6 \\
1
\end{array}\right]\right) d\left(b, 2,\left[\begin{array}{l}
2 \\
1 \\
3 \\
1
\end{array}\right]\right), \quad f\left(c,\left[\begin{array}{l}
5 \\
2 \\
0
\end{array}\right]\right) d\left(b, 2,\left[\begin{array}{l}
2 \\
1 \\
3 \\
1
\end{array}\right]\right), \\
f\left(b,\left[\begin{array}{l}
2 \\
1 \\
3 \\
1
\end{array}\right]\right) d\left(c, 1,\left[\begin{array}{l}
5 \\
2 \\
0
\end{array}\right]\right), \quad d\left(b, 2,\left[\begin{array}{l}
2 \\
1 \\
3 \\
1
\end{array}\right]\right) \wedge d\left(c, 1,\left[\begin{array}{l}
5 \\
2 \\
0
\end{array}\right]\right) .
\end{gathered}
$$

Lemma 4.2. Let $c=(a, e, \vec{x})$ be a reduced 1 -cell. For any cell s such that $[c] \leq[s]$, $e \in s$. Furthermore, $D_{a, i}(s)=D_{a, i}(c)=\vec{x}_{i}$ for each $i \in\{0, \ldots, \operatorname{deg}(a)-1\}$.

Proof. By the definition of $\leq, s$ must contain $e$. The lemma then follows from the definition of $\sim$.

For a vertex $a$ and an $a$-vector $\vec{x}$ with $|\vec{x}|=n$, define the 0 -form $f(a, \vec{x})$ : $C_{*}\left(U D^{n} T\right) \rightarrow \mathbb{Z} / 2 \mathbb{Z}$ by:

$$
c \mapsto \begin{cases}1 \quad & \text { if } D_{a, i}(c)=x_{i} \text { for all } i \in\{0, \ldots, \operatorname{deg}(a)-1\}, \text { or } \\ & \bar{D}_{a, i}(c)=x_{i} \text { for all } i \in\{0, \ldots, \operatorname{deg}(a)-1\}, \\ 0 \quad & \text { otherwise, }\end{cases}
$$

extended linearly to all cellular chains. The constant function 1 is a 0 -form: for any vertex $a \neq *$ of degree 1 in $T, f(a, 0)=1$.

Now we define $k$-forms. Let $c=(a, e, \vec{x})$ be a reduced 1-cell. Define the basic 1 -form $d c=d(a, e, \vec{x}): C_{*}\left(U D^{n} T\right) \rightarrow \mathbb{Z} / 2 \mathbb{Z}$ by:

$$
c^{\prime} \mapsto \begin{cases}1 & \text { if } e \in c^{\prime} \text { and } f(a, \vec{x})\left(\left[c^{\prime}\right]\right)=1, \\ 0 & \text { otherwise, }\end{cases}
$$


and extend linearly to all cellular chains. In general, a basic $k$-form is

$$
f(a, \vec{x}) d c_{1} \wedge \cdots \wedge d c_{k},
$$

where $f(a, \vec{x})$ is a 0 -form as above and each of $c_{1}, \ldots, c_{k}$ is a distinct reduced 1 -cell. Here, the wedge product represents conjunction: for $[c] \in H_{*}\left(B_{n} T\right)$, the $k$-form sends $[c]$ to 1 if and only if $[c] \mapsto 1$ under $f(a, \vec{x})$ and under each $d c_{i}$. If not all of the $c_{i}$ are distinct, then the $k$-form is identically the 0 -function.

As an example of forms, see Figure 3.

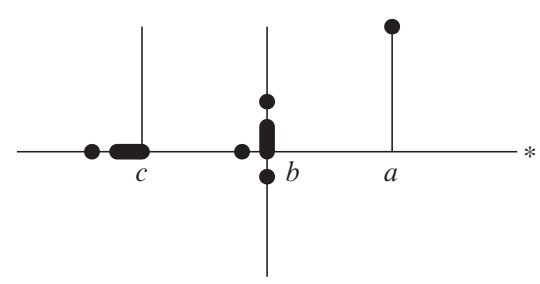

Figure 3. This figure depicts the 2-cell $s$ used in Example 4.1.

Thus defined, forms have the following interpretation for cohomology:

Proposition 4.3 (Forms and cohomology). Let T be a tree. Fix a Morse T-embedding. Then under the induced Morse matching:

(1) If $c$ is a reduced 1-cell, then $\phi_{[c]}=d c$. In particular, if $c$ is a critical 1-cell, then $c^{*}=[d c]$.

(2) If $c_{1}, \ldots, c_{k}$ are reduced 1 -cells, then

$$
\left[\phi_{\left[c_{1}\right]}\right] \cup \ldots\left[\phi_{\left[c_{k}\right]}\right]=\left[d c_{1} \wedge \cdots \wedge d c_{k}\right] .
$$

In particular, if $c_{1}, \ldots, c_{k}$ are critical 1 -cells, then

$$
c_{1}^{*} \cup \cdots \cup c_{k}^{*}=\left[d c_{1} \wedge \cdots \wedge d c_{k}\right] .
$$

(3) If $c_{1}, \ldots, c_{k}$ are reduced 1 -cells such that $\left\{\left[c_{1}\right], \ldots,\left[c_{k}\right]\right\}$ has no upper bound or contains a repeated element, then

$$
\left[d c_{1} \wedge \cdots \wedge d c_{k}\right]=[0] .
$$

Proof. Part (3) and that $c^{*}=\left[\phi_{[c]}\right]$ follow from Theorem 3.6. The remaining statements follow from chasing definitions.

Now that we have defined forms, we wish to define differentials for these forms. We will see in Proposition 4.6 that coboundaries and differentials coincide. Before defining differentials, though, we give a motivating theorem, Theorem 4.5 , for why coboundaries are important. To state the theorem, we need a few more definitions. 
Construct a simplicial complex $K$ from $U D^{n} T$ as follows. First define a simplicial complex $K^{\prime \prime}$. The vertex set of $K^{\prime \prime}$ corresponds to distinct equivalence classes of 1-cells $[c]$. Vertices $\left[c_{1}\right], \ldots,\left[c_{k}\right]$ span a $(k-1)$-simplex in $K^{\prime \prime}$ if and only if $\left\{\left[c_{1}\right], \ldots,\left[c_{k}\right]\right\}$ has an upper bound $[s]$, and is labelled by $[s]$. Note the labels on the faces of $K^{\prime \prime}$ induce an injective map from equivalence classes of cells in $U D^{n} T$ to $\Lambda\left(K^{\prime \prime}\right)$ (see Theorem 3.5). Let $K^{\prime}$ denote the $\left(\left\lfloor\frac{n}{2}\right\rfloor-1\right)$-skeleton of $K^{\prime \prime}$; then $\Lambda\left(K^{\prime}\right)=\Lambda\left(K^{\prime \prime}\right) / I^{\prime \prime}$, where $I^{\prime \prime}$ is the ideal of $\Lambda\left(K^{\prime \prime}\right)$ generated by all simplices of dimension greater than $\left\lfloor\frac{n}{2}\right\rfloor-1$. Finally, define the complex $K$ to be the subcomplex of $K^{\prime}$ where the only vertices left correspond to equivalence classes of 1-cells [c] with non-extraneous reduced representatives. Then $\Lambda(K)=\Lambda\left(K^{\prime}\right) / I^{\prime}$, where $I^{\prime}$ is the ideal of $\Lambda\left(K^{\prime}\right)$ generated by equivalence classes of extraneous reduced 1-cells (see Section 3.2).

Definition 4.4 (Necessary forms and cells). Let $\omega=f(a, \vec{x}) d c_{1} \wedge \cdots \wedge d c_{k}$ be a $k$-form. The $k$-form $\omega$ is necessary if:

(1) $k<\lfloor n / 2\rfloor{ }^{1}$,

(2) there exists an edge $e \in T$ such that $(a, e, \vec{x})$ is a non-extraneous reduced 1-cell,

(3) the set $\left\{\left[c_{1}\right], \ldots,\left[c_{k}\right],[(a, e, \vec{x})]\right\}$ has an upper bound $[s]$, and

(4) the edge $e$ is the unique respectful edge in the reduced representative $s$ of $[s]$.

The reduced 1-cell $(a, e, \vec{x})$ is the necessary reduced 1-cell for $\omega$, and is called necessary.

We leave it as an exercise for the reader to verify that there exists a unique necessary reduced 1-cell for a given necessary form.

For an arbitrary $k$-form $\omega$, the $(k+1)$-chain which is the sum of all equivalence classes in the support of the coboundary of $\omega$ will be called the coboundary support chain for $\omega$.

Theorem 4.5 (Presentation for cohomology; [14], Theorem 4.5). We have that

$$
H^{*}\left(B_{n} T\right) \cong \Lambda(K) / I,
$$

where I is the ideal of $\Lambda(K)$ generated by all coboundary support chains for necessary forms, viewed as cochains. The isomorphism is induced by the injective map from equivalence classes of cells in $U D^{n} T$ to $\Lambda\left(K^{\prime \prime}\right)$.

Proof. This theorem is almost a rewording of Farley's Theorem 4.5 from [14]. The difference is that Farley does not define the ideals $I, I^{\prime}$, and $I^{\prime \prime}$, but instead views $H^{*}\left(B_{n} T\right)$ as isomorphic to a quotient of $\Lambda\left(K^{\prime \prime}\right)$. A statement of the result presented here which more closely resembles Farley's theorem is that

$$
H^{*}\left(B_{n} T\right) \cong\left(\left(\Lambda\left(K^{\prime \prime}\right) / I^{\prime \prime}\right) / I^{\prime}\right) / I .
$$

\footnotetext{
${ }^{1}$ The reason for this dimension restriction is that the simplicial complex $K$ of Theorem 4.5 has no faces in dimension $\left\lfloor\frac{n}{2}\right\rfloor$ or larger; see Theorem 3.6.
} 
That we may quotient by the ideal $I^{\prime \prime}$ follows from Theorem 3.6: cohomology is trivial in all dimensions greater that $\left\lfloor\frac{n}{2}\right\rfloor$.

That we may then quotient by $I^{\prime}$ is more difficult to see. Clearly $I^{\prime}$ is an ideal of $\Lambda\left(K^{\prime}\right)$. Let $c=(a, e, \vec{x})$ be an extraneous 1-cell. If the edge $e$ of $c$ is such that $\tau(e)$ is essential, then this text and Farley agree: both quotient by the ideal generated by $\partial f(a, \vec{x})$. Now consider if the edge $e$ of $c$ is such that $\tau(e)$ is not essential. We may still talk about directions from $\tau(e)$, but there are at most 2 directions. Let $x_{0}:=D_{\tau(e), 0}(c)$, and let $x_{1}:=D_{\tau(e), 1}(c)$ if a second direction from $\tau(e)$ exists or 0 otherwise. Even though the cell $c$ is not necessarily uniquely determined from the information $(\tau(e), e, \vec{x})$, its equivalence class $[c]$ is. For the purposes of this proof, we will abuse notation and write $[c]=[(\tau(e), e, \vec{x})]$. For example, consider the cell $c$ in Figure 4 with edge $e$. If exactly one of the non-filled-in vertices is an element of $c$, then $[c]=\left[\left(\tau(e), e,\left[\begin{array}{l}1 \\ 4\end{array}\right]\right)\right]$. As the tuple $\left(\tau(e), e,\left[\begin{array}{l}1 \\ 4\end{array}\right]\right)$ does not depend on which of the two non-filled-in vertices is an element of $c, c$ is not uniquely determined by it.

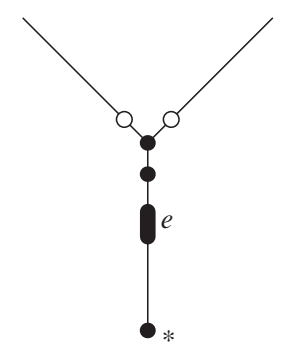

Figure 4. An extraneous 1-cell $c$ whose edge $e$ is such that $\tau(e)$ is not essential.

Consider the $\tau(e)$-vector $\vec{x}^{\prime}$, where $x_{0}^{\prime}:=x_{0}-1$ and $x_{1}^{\prime}:=x_{1}+1$. If $x_{0}=0$, or there are less than $x_{1}^{\prime}$ vertices in direction 1 from $\tau(e)$ in all of $T$, then there are no 1-cells $c^{\prime}$ satisfying $\left[c^{\prime}\right]=\left[\left(\tau(e), e, \vec{x}^{\prime}\right)\right]$. In this case, Farley includes $[c]$ in the generating set of his quotient ideal, as we have done here. Otherwise, tracing Farley's definitions yields that the Farley generating set does not include $[c]$, but instead includes the chain $[c]+\left[\left(\tau(e), e, \vec{x}^{\prime}\right)\right]$. In the quotient, $[c]$ and $\left[\left(\tau(e), e, \vec{x}^{\prime}\right)\right]$ are equivalent. Inducting on the value $x_{0}$, we see that indeed $[c]$ is equivalent to 0 in the quotient. The ideal $I^{\prime}$ is thus precisely the subideal of Farley's ideal corresponding to these chains.

Carefully tracing Farley's definitions and the definition of necessary show that the remaining generating cochains of Farley's ideal precisely coincide with coboundary support chains for necessary forms. Note that in [14], the convention that $\tau(e)<\iota(e)$ is switched.

Let $\omega=f(a, \vec{x}) d c_{1} \wedge \cdots \wedge d c_{k}$ be a necessary cochain. Necessary cochains were defined so that each term in the coboundary support chain of $\omega$ has the form 
$d c_{0} \wedge d c_{1} \wedge \cdots \wedge d c_{k}$, where each $c_{i}$ is a non-extraneous critical 1-cell for $i=$ $0,1, \ldots, k$. We leave this to the reader to verify. In particular, since each $c_{i}$ is non-extraneous and $k<\lfloor n / 2\rfloor$, every term in the coboundary support chain of $\omega$ represents a nontrivial element of $\Lambda(K)$.

We already know that cohomology is generated by duals of critical cells. Theorem 4.5 tells us that we may effectively ignore all extraneous reduced 1-cells. From now on, all reduced cells will be assumed to be non-extraneous unless otherwise stated.

Theorem 4.5 also tells us that coboundaries of necessary forms show us how to rewrite duals of noncritical cells in terms of our Morse basis. The isomorphism in the theorem takes a characteristic function $\phi_{[c]}$ of a cell $c$ to the element of $\Lambda(K) / I$ corresponding to $[c]$.

We wish to understand coboundaries of necessary forms. To do so, we define differentials of forms, and show that our differential coincides with the operation of coboundary.

Define the differential of a form as follows. For a basic 0 -form $f(a, \vec{x})$, define

$$
d f(a, \vec{x})=\sum(f(a, \vec{x})(\partial c)) d c,
$$

where the sum runs over all reduced 1-cells $c$, and $\partial c$ is the CW-boundary of $c$ as a cellular chain. We are left with an expression of $d f(a, \vec{x})$ as a sum of basic 1-forms, all of which have 1 as the leading 0 -form. Note that $d 1=0$. For a basic $k$-form $\omega=f(a, \vec{x}) d c_{1} \wedge \cdots \wedge d c_{k}$, define

$$
d \omega:=d\left(f(a, \vec{x}) \wedge d c_{1} \wedge \cdots \wedge d c_{k}\right)=(d f(a, \vec{x})) \wedge d c_{1} \wedge \cdots \wedge d c_{k} .
$$

Extend linearly to all $k$-forms. Since $d 1=0$, it is clear that $d^{2}=0$.

We are now ready to prove the relationship between differentials and coboundaries:

Proposition 4.6 (Coboundary and differential). The differential operation d precisely coincides with the coboundary operation.

Proof. To compute the coboundary $\delta \omega$ of an $k$-cochain $\omega=f(a, \vec{x}) \wedge d c_{1} \wedge \cdots \wedge d c_{k}$, we consider all $\sim$-equivalence classes of $(k+1)$-cells in $U D^{n} T_{\min }$. Let $[s]$ be an equivalence class of $(k+1)$-cells, and let $s \in[s]$.

Consider a face $s^{\prime}$ of $s$. The face $s^{\prime}$ corresponds to replacing some edge $f$ of $s$ with one of its endpoints. For any $i \in\{1, \ldots, k\}$, we claim that if $d c_{i}(s)=0$, then $d c_{i}\left(s^{\prime}\right)=0$. For, let $e_{i}$ denote the edge of $c_{i}$. If $e_{i} \notin s$, then $e_{i} \notin s^{\prime}$. Consider if $e_{i} \in s$. If $e_{i} \notin s^{\prime}$ (that is, if $\left.f=e_{i}\right)$, then $d c_{i}\left(s^{\prime}\right)=0$ and there is nothing to prove. If $e_{i} \in s^{\prime}$ (that is, if $f \neq e_{i}$ ), then since $T$ is a tree both endpoints of $f$ are in the same direction from $\tau(e)$. In particular, the number of vertices in each direction from $\tau(e)$ is the same for both $s$ and $s^{\prime}$. If $e_{i} \in s$, but $d c_{i}(s)=0$, then by definition the number of vertices of $s$ (and thus $s^{\prime}$ ) in some direction - say $j$ - from $\tau\left(e_{i}\right)$ is not equal to the number of vertices in $c_{i}$ in direction $j$ from $\tau\left(e_{i}\right)$; thus, $d c_{i}\left(s^{\prime}\right)=0$. 
We have proven that for any $i \in\{1, \ldots, k\}$, if $d c_{i}(s)=0$, then for any face $s^{\prime}$ of $s$, we will have $d c_{i}\left(s^{\prime}\right)=0$. By the definition of coboundary, $\delta \omega(s)=1$ if and only if the sum of $\omega$ evaluated on the $2^{k+1} k$-faces of $s$ is 1 in $\mathbb{Z} / 2 \mathbb{Z}$. Thus, if there exists an $i \in\{1, \ldots, k\}$ such that $d c_{i}(s)=0$, then $\delta \omega(s)=0=d \omega(s)$.

Assume that $d c_{i}(s)=1$ for each $i \in\{1, \ldots, k\}$. Then $s$ contains the edge $e_{i}$ of the cell $c_{i}$ for each $i$. If $e_{i}=e_{j}$ for some $i \neq j$, then since $d c_{i}(s)=d c_{j}(s)=1$, it must be that $c_{i}=c_{j}$. But then $d c_{i} \wedge d c_{j}=d c_{i}^{2}=0$, and again both $\delta \omega(s)=0$ and $d \omega(s)=0$. Now assume the edges $e_{i}$ are all distinct. Let $e$ denote the edge of $s$ which is not one of the $e_{i}$. Consider again a face $s^{\prime}$ of $s$ corresponding to replacing some edge $f$ of $s$ with one of its endpoints. If $f \neq e$, then $f=e_{i}$ for some $i$, so $d c_{i}$ and therefore $\omega$ evaluated on the given face will be 0 . Let $s^{\prime}$ and $s^{\prime \prime}$ be the two faces of $s$ corresponding to replacing $e$ with one of its endpoints. Thus, since $d c_{i}(s)=d c_{i}\left(s^{\prime}\right)=d c_{i}\left(s^{\prime \prime}\right)=1$ for each $i \in\{1, \ldots, k\}$,

$$
\delta \omega(s)=\omega\left(s^{\prime}\right)+\omega\left(s^{\prime \prime}\right)=f(a, \vec{x})\left(s^{\prime}\right)+f(a, \vec{x})\left(s^{\prime \prime}\right) .
$$

Also, by the definition of differential,

$$
d \omega(s)=f(a, \vec{x})(\partial s) d s(s)=f(a, \vec{x})\left(s^{\prime}\right)+f(a, \vec{x})\left(s^{\prime \prime}\right) .
$$

In this final case, we have again shown that $\delta \omega(s)=d \omega(s)$. Since $[s]$ was chosen arbitrarily, $\delta \omega=d \omega$ as desired.

The proof of Proposition 4.6 actually gives us some computational techniques for considering differentials. In particular, because of the last equation in the proof, we have the following scholium:

Corollary 4.7 (Restricting the differential). Let $f(a, \vec{x})$ be a basic 0 -form and let $s$ be a reduced $k$-cell.

(1) If $f(a, \vec{x})(\partial s)=1$ then $s$ contains an edge $e$ with $\tau(e)=a$.

(2) For each $i \in\{0, \ldots, \operatorname{deg}(a)-1\}$, let $x_{i}^{\prime}:=D_{a, i}(s)$, and let $d$ be the direction from a along $e$. If $f(a, \vec{x})(\partial s)=1$ then either $\vec{x}=\vec{x}^{\prime}$ or $\vec{x}^{\prime}$ differs from $\vec{x}$ by the subtraction of 1 in the $0^{\text {th }}$ entry and the addition of 1 in the $d^{\text {th }}$ entry.

More can be said about $d \omega$ when $\omega=f(a, \vec{x}) d c_{1} \wedge \cdots \wedge d c_{k}$ is a basic $k$-form. Many of the 1 -forms in the sum used to define $d(f(a, \vec{x}))$ multiply to 0 when wedged with $d c_{1} \wedge \cdots \wedge d c_{k}$. That is, many of the terms of the expansion of $d \omega$ with respect to the definition of $d f(a, \vec{x})$ are trivial. We define an annihilator function to get rid of the trivial terms.

Definition 4.8 (Annihilator). Let $c_{1}, \ldots, c_{k}$ be reduced 1-cells. Define an annihilator function $A_{c_{1}, \ldots, c_{k}}$ on 1-forms as follows. Let $d(a, e, \vec{x})$ be a basic 1form. Then the annihilator acts either as the identity or the 0 function on $d(a, e, \vec{x})$ : $A_{c_{1}, \ldots, c_{k}}(d(a, e, \vec{x}))=d(a, e, \vec{x})$ if and only if 
- the equivalence classes $\left[c_{1}\right], \ldots,\left[c_{k}\right]$, and $[(a, e, \vec{x})]$ are all distinct, and

- the set of equivalence classes $\left\{\left[c_{1}\right], \ldots,\left[c_{k}\right],[(a, e, \vec{x})]\right\}$ has an upper bound; otherwise, $A_{c_{1}, \ldots, c_{k}}(d(a, e, \vec{x}))=0$. Extend $A_{c_{1}, \ldots, c_{k}}$ linearly to all 1-forms.

Corollary 4.9 (Coboundaries as annihilators). We have that

$$
\delta \omega=d \omega=A_{c_{1}, \ldots, c_{k}}(d f(a, \vec{x})) \wedge d c_{1} \wedge \cdots \wedge d c_{k} .
$$

Proof. This follows from the definition of the annihilator $A_{c_{1}, \ldots, c_{k}}$, Proposition 4.6, and part (3) of Proposition 4.3.

We refer the reader to the recent paper [14] for more information on coboundaries and cohomology presentations.

\section{Exterior face algebra structures on cohomology}

Recall that a linear tree is one in which there exists an embedded line segment containing every essential vertex. Consider the following theorem:

Theorem 5.1. Let $T$ be a tree. If $T$ is linear or $n \leq 3$, then $H^{*}\left(B_{n} T ; \mathbb{Z} / 2 \mathbb{Z}\right)$ is an exterior face algebra.

Proof. A tree braid group $B_{n} T$ is a right-angled Artin group (see Section 8 for a definition) if and only if $T$ is linear or $n \leq 3$ ([7] proves linear trees are right-angled Artin; [17] and [15] prove $B_{n} T$ is free if $n \leq 3$; [16] proves the only if direction). For any right-angled Artin group, the cohomology ring is an exterior face algebra [6].

Let $T_{\min }$ be the 'minimal' nonlinear tree of Figure 1: $T_{\min }$ has exactly 4 essential vertices, each of degree 3 , such that not all 4 essential vertices lie on an embedded line segment. In [16], it was shown that $H^{*}\left(B_{4} T_{\min } ; \mathbb{Z} / 2 \mathbb{Z}\right)$ is an exterior face algebra $\Lambda(\Delta)$; Figure 5 shows the complex $\Delta$. In this section, our goal is to expand the easy observations in Theorem 5.1 to show that the cohomology of a four, or even five, strand tree braid group is an exterior face algebra holds in general:

Theorem 5.2 (Exterior face algebra structure on 4 and 5 strand cohomology). Let $T$ be a finite tree. For $n=4$ or $5, H^{*}\left(B_{n} T ; \mathbb{Z} / 2 \mathbb{Z}\right)$ is an exterior face algebra. The simplicial complex $\Delta$ defining the exterior face algebra structure is unique and at most 1-dimensional.

We will comment on more general tree braid groups at the end of the section. As our coefficients for cohomology are always in $\mathbb{Z} / 2 \mathbb{Z}$, we suppress this in the notation from now on. Even though a 1-dimensional simplicial complex (for instance, $\Delta$ ) is a graph, we will continue to refer to them as simplicial complexes to maintain 


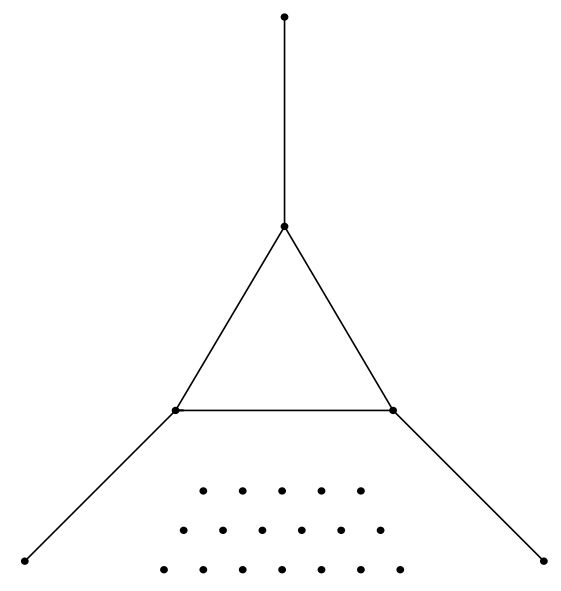

Figure 5. The simplicial complex $\Delta$ giving the exterior face algebra structure on $H^{*}\left(B_{4} T_{\min } ; \mathbb{Z} / 2 \mathbb{Z}\right)$.

the distinction between defining complexes for exterior face algebras and graphs underlying graph braid groups.

Some of the statements of Theorem 5.2 are immediate, and we prove them now.

Lemma 5.3 (Uniqueness and the dimension bound). Let $T$ be a finite tree. For $n=4$ or 5 , if $H^{*}\left(B_{n} T\right)$ is an exterior face algebra then the simplicial complex $\Delta$ defining the exterior face algebra structure is unique and at most 1-dimensional.

Proof. By Theorem 3.6 and a dimension computation for $U D^{n} T$ in [15], cohomology is trivial in all dimensions greater than $\lfloor n / 2\rfloor=2$. Thus, if $H^{*}\left(B_{n} T\right)$ is an exterior face algebra corresponding to some simplicial complex $\Delta, \Delta$ must be at most (2-1)dimensional, by the shift of indices in the definition of exterior face algebras. By Theorem 2.3, such a complex $\Delta$ is unique.

To prove Theorem 5.2, it remains to show that $H^{*}\left(B_{n} T\right)$ is an exterior face algebra. We will spend much of the remainder of this section proving this, via a series of lemmas. In essence, we will define a 1-dimensional simplicial complex $\Delta$ and a homomorphism $\Psi: \Lambda(\Delta) \rightarrow H^{*}\left(B_{n} T\right)$, and then show the homomorphism is an isomorphism. The bulk of our effort will be in defining $\Delta$, via a change of basis for $H^{*}\left(B_{n} T\right)$.

Until the end of the proof of Theorem 5.2, we will assume that $n \in\{4,5\}$.

5.1. Two computational lemmas. In this subsection we establish two computational lemmas to be used throughout the remainder of this section. 
Lemma 5.4 (Necessary 0-forms). Let $c=(a, e, \vec{x})$ be a reduced noncritical 1-cell. Then $f(a, \vec{x})$ is necessary, $c$ is necessary for the 0 -form $f(a, \vec{x})$, and $f(a, \vec{x})$ is the unique 0 -form for which $c$ is necessary.

Proof. This is a direct consequence of the definitions of necessary and $f(a, \vec{x})$.

Lemma 5.5 (Necessary 1-forms). Let $c=(a, e, \vec{x})$ and $c_{1}=\left(b, e_{1}, \vec{y}\right)$ be critical 1-cells. If $\omega:=f(a, \vec{x}) d c_{1}$ is necessary and $c$ is the necessary reduced 1-cell for $\omega$, then:

(1) the direction from $b$ to $a$ is 0 ,

(2) the direction $\alpha$ from a to $b$ satisfies $0<\alpha<d$, and

(3) $\alpha$ is the unique direction between 0 and $d$ for which $x_{\alpha} \neq 0$.

Proof. Let $[s]$ be the least upper bound of $\left\{[c],\left[c_{1}\right]\right\}$. By the definition of $\leq, s$ must contain the edges $e$ and $e_{1}$. But the only edges of $s$ are $e$ and $e_{1}$, for if $s$ contains any other edges, replacing each with one of its endpoints yields a smaller upper bound.

Since $e$ is disrespectful in $c$, there is a vertex of $c$ between $\tau(e)$ and $\iota(e)$ in the order on vertices, i.e. in direction $d_{0}$ from $a$, where $0<d_{0}<d$. By Lemma 4.2, $s$ still has a vertex or edge in direction $d_{0}$ from $a$.

We finish the proof by contradiction. If the direction from $b$ to $a$ is nonzero, then the direction from $a$ to $b$ must be 0 (since $T$ is a tree) - in particular, $e_{1}$ is not in direction $d_{0}$ from $a$. If $\alpha$ does not satisfy $0<\alpha<d$, then $\alpha \neq d_{0}$, and again $e_{1}$ is not in direction $d_{0}$ from $a$. If $c$ has two nonzero directions less than $d$ such that $c$ has vertices in both directions from $a$, then without loss of generality we may assume $d_{0}$ is such that $d_{0} \neq \alpha$, and again $e_{1}$ is not in direction $d_{0}$ from $a$.

Thus, if any of the conclusions of this lemma do not hold, then $s$ has a vertex in direction $d_{0}$ from $a$. Assume without loss of generality that $s$ is reduced, and let $v$ be the least vertex in direction $d_{0}$ from $a$. Then $v$ must be blocked in $s$ by either $*, e_{1}$, or $e$. Since $d_{0} \neq 0, v$ cannot be blocked by $*$. Since $d_{0} \neq \alpha, v$ cannot be blocked by $e_{1}$. So, $v$ must be blocked by $e$. This makes $e$ disrespectful in $s$, since $0<d_{0}<d$. This contradicts the definition of necessary, and proves the lemma.

5.2. Towards changing bases. In this subsection, we describe why we need to change bases to find the exterior face algebra structure on cohomology. We also define many matrices, some associated to necessary forms and some to critical 1cells, which we will use in the next section to define our change of basis matrix.

Fix a Morse $T$-embedding, so that we have a classification of critical cells in $T$. Consider the finite simplicial complex $\Delta^{\prime}$, defined as follows. Let the vertex set of $\Delta^{\prime}$ be identified with the set $\left\{c^{*} \mid c\right.$ a critical 1-cell $\}$. A set of vertices $\left\{c_{1}^{*}, c_{2}^{*}\right\}$ span a 1-simplex, labelled $c_{1}^{*} \cup c_{2}^{*}$, if and only if $c_{1}^{*} \cup c_{2}^{*}$ is nontrivial.

Let $\Psi^{\prime}: \Lambda\left(\Delta^{\prime}\right) \rightarrow H^{*}\left(B_{n} T\right)$ be the map which takes an element of $\Lambda\left(\Delta^{\prime}\right)$ corresponding to a vertex of $\Delta^{\prime}$ labelled $c^{*}$ and maps it to the $i$-cohomology class $c^{*}$. Since 
critical 1-cells form a free basis for $H^{1}\left(B_{n} T\right)$, the map extends to all of $H^{1}\left(B_{n} T\right)$. By the definition of $\Delta^{\prime}, \Psi^{\prime}$ is surjective onto $H^{1}\left(B_{n} T\right)$ and moreover extends linearly to a surjective homomorphism, since $H^{1}\left(B_{n} T\right)$ generates all of $H^{*}\left(B_{n} T\right)$ (see Theorem 3.6).

If $\Psi^{\prime}$ were injective, then $\Psi^{\prime}$ would be the desired isomorphism. Recall that a critical 2-cell $s$ uniquely determines the pair $\left\{c_{1}, c_{2}\right\}$ of critical 1-cells for which it is an upper bound (Theorem 3.6). As critical 2-cells form a basis for 2-dimensional cohomology, $\Psi^{\prime}$ is injective if and only if, for every pair $\left\{c_{1}, c_{2}\right\}$ of critical 1-cells which has a least upper bound $[s]$, we may find a representative $s \in[s]$ such that $s$ is a critical 2-cell. Unfortunately, though, this is not the case, and $\Psi^{\prime}$ is not injective.

Our goal is to modify the Morse generating set of $H^{*}\left(B_{n} T\right)$ to address these cases. We will define a new simplicial complex $\Delta$ using this new generating set so that the corresponding map $\Psi: \Lambda(\Delta) \rightarrow H^{*}\left(B_{n} T\right)$ will be an isomorphism.

To modify the generating set of duals of critical 1-cells, we need to specify a change of basis. Since duals of critical 1-cells freely generate $H^{1}\left(B_{n} T\right)$, we may introduce a vector-theoretic interpretation of $H^{1}\left(B_{n} T\right)$. Then, using this vectortheoretic interpretation, we specify an invertible change of basis matrix $M$. The matrix $M$ will be a product of invertible matrices, one for each critical cell, in a particular order, as we will see.

Define $<_{r}$ to be a (usually non-unique) total order on reduced 1-cells $(a, d, \vec{x})$ induced by lexicographically ordering the triple $\left(a,-x_{0}, d\right)$. Note we are ordering all reduced 1-cells here, not just critical ones. Let $\mathrm{rm}$ be the number of reduced 1-cells, $s m$ the number of critical 1-cells, and $t m$ the number of non-critical reduced 1-cells, so $r m=s m+t m$. Define a map $r i$ on reduced 1-cells, so that for a reduced 1-cell $c$, its image $\operatorname{ri}(c) \in\{1, \ldots, M\}$ is its index in the total order $<_{r}$, so that the $<_{r}$-smallest reduced 1-cell has index 1, the second smallest has index 2, etc. Also define maps $s i(c)$ and $t i(c)$ on critical and non-critical reduced 1-cells respectively, so that for a critical (respectively, noncritical) 1-cell $c, \operatorname{si}(c)$ is its index among critical (respectively, noncritical) cells in the total order $<_{r}$. Ergo, the $<_{r}$-smallest critical 1 -cell maps to 1 under $c i$, the second smallest to 2 , etc.

There is a bijection between reduced 1-cells and the standard basis vectors for $\mathbb{F}_{2}^{r} m$, where a reduced 1-cell $c$ corresponds to the vector $\vec{v}_{c}$ consisting of all $0 \mathrm{~s}$ except a 1 in the $r i(c)^{\text {th }}$ row. By Theorems 3.3 and 3.6, this bijection induces a surjective homomorphism from $\mathbb{F}_{2}^{r m}$ to each of the rings $H_{1}\left(B_{n} T\right)$ and $H^{1}\left(B_{n} T\right)$. The surjection is an isomorphism on the $s m$-dimensional subspace corresponding to the critical 1-cells, where for a critical cell $c, \vec{v}_{c}$ is mapped to $[c]$ or $c^{*}$, respectively.

Theorem 4.5 tells us that, if we want to rewrite our basis for cohomology to reduce the number of relations in our presentation, then we need to focus on necessary $k$ forms. So, we use these vector representatives of critical 1-cells to associate to each necessary $k$-form $\omega$ a matrix $M_{\omega}$.

Let $\omega=f(a, \vec{x}) d c_{1} \wedge \cdots \wedge d c_{k}$ be a necessary $k$-form with $c$ the necessary reduced 1-cell for $\omega$. Consider the annihilator portion $A_{c_{1}, \ldots, c_{k}}(d f(a, \vec{x}))$ of $d \omega$. Let $\vec{u}_{\omega} \in \mathbb{F}_{2}^{r m}$ be the vector whose nonzero entries exactly correspond to nonzero terms 
of $A_{c_{1}, \ldots, c_{k}}(d f(a, \vec{x}))$. That is, $\left(u_{\omega}\right)_{r i\left(c^{\prime}\right)}=1$ if and only if $d c^{\prime}$ is a nonzero term of $A_{c_{1}, \ldots, c_{k}}(d f(a, \vec{x}))$. Define the $r m \times r m$ matrix $M_{\omega}$ to be the identity matrix $I_{m}$, but with the $r i(c)^{\text {th }}$ column replaced by $\vec{u}_{\omega}$.

Lemma 5.6. For any necessary $k$-form $\omega=f(a, \vec{x}) d c_{1} \wedge \cdots \wedge d c_{k}$, the necessary reduced 1-cell $c$ for $\omega$ is the $<_{r}$-smallest reduced 1 -cell $c^{\prime}$ such that $d c^{\prime}$ appears as a nonzero term in $A_{c_{1}, \ldots, c_{k}}(d f(a, \vec{x}))$.

Note this lemma holds for arbitrary $n$, not just $n=4$ and 5 .

In Convention 5.8, we will slightly modify the definition of $<_{r}$ in the cases $n=4$ or 5 . One consequence will be that, for a necessary 1 -form whose associated necessary critical 1-cell $c$ is exceptional of Type I (to be defined soon), this lemma and its corollary will not hold - in fact, $M_{\omega}$ will be upper triangular. The modification will not affect our applications of these results, though.

Proof of Lemma 5.6. Let $c=(a, e, \vec{x})$ be the reduced 1-cell for the $\omega$. Let $c^{\prime} \neq c$ be any other reduced 1-cell such that $d c^{\prime}$ appears nontrivially in $d f(a, \vec{x})$. By restricting the differential (Corollary 4.7), $c^{\prime}$ must have an edge $e^{\prime}$ with $\tau\left(e^{\prime}\right)=a$. By the definition of differential, we know that $f(a, \vec{x})\left(\partial c^{\prime}\right)=1$. Let $c_{\iota}^{\prime}$ and $c_{\tau}^{\prime}$ denote the faces of $c^{\prime}$, corresponding to replacing $e^{\prime}$ with $\iota\left(e^{\prime}\right)$ and $\tau\left(e^{\prime}\right)$, respectively. Then exactly one of $f(a, \vec{x})\left(c_{l}^{\prime}\right)$ or $f(a, \vec{x})\left(c_{\tau}^{\prime}\right)$ is 1 . For each direction $i$ from $a$,

$$
D_{a, i}\left(c_{l}^{\prime}\right)=\bar{D}_{a, i}\left(c_{l}^{\prime}\right)=D_{a, i}\left(c^{\prime}\right),
$$

and

$$
D_{a, i}\left(c_{\tau}^{\prime}\right)=\bar{D}_{a, i}\left(c_{\tau}^{\prime}\right)=\bar{D}_{a, i}\left(c^{\prime}\right) .
$$

We need to show that $c<_{r} c^{\prime}$. If $f(a, \vec{x})\left(c_{\tau}^{\prime}\right)=1$ then for $i=0$ we have $D_{a, 0}\left(c^{\prime}\right)=\bar{D}_{a, 0}\left(c^{\prime}\right)=x_{0}-1$, so $c<_{r} c^{\prime}$. It remains to consider the case when $f(a, \vec{x})\left(c_{\iota}^{\prime}\right)=1$. Then $D_{a, i}\left(c^{\prime}\right)=x_{i}=D_{a, i}(c)$, and in particular, $c^{\prime}=\left(a, e^{\prime}, \vec{x}\right)$.

By the definition of necessary, $c$ is such that:

(1) the set $\left\{\left[c_{1}\right], \ldots,\left[c_{k}\right],[c]\right\}$ has an upper bound $[s]$, and

(2) the edge $e$ is respectful in the reduced representative $s$ of $[s]$.

Let $d>0$ be the direction from $a$ along $e$ and let $d^{\prime}>0$ be the direction from $a$ along $e^{\prime}$. Since $D_{a, i}\left(c^{\prime}\right)=x_{i}$ for each $i$ and $e^{\prime} \in c^{\prime}, D_{a, d^{\prime}}(c)=D_{a, d^{\prime}}\left(c^{\prime}\right) \geq 1$. Unless $d=d^{\prime}$ so that $c=c^{\prime}, s$ must have a vertex in direction $d^{\prime}$ from $a$. Since $s$ is reduced, that vertex must be blocked by $e$ in $s$. Since $e$ is respectful in $s, d^{\prime}>d$. Thus, $c<_{r} c^{\prime}$.

Corollary 5.7 ( $M_{\omega}$ is lower triangular). For any necessary $k$-form $\omega$, the matrix $M_{\omega}$ is lower triangular and invertible.

Proof. That $c<_{r} c^{\prime}$ for any other $c^{\prime}$ for which $d c^{\prime}$ appears nontrivially in the annihilator $A_{c_{1}, \ldots, c_{k}}(d f(a, \vec{x}))$ makes $M_{\omega}$ a lower triangular matrix. By the definition 


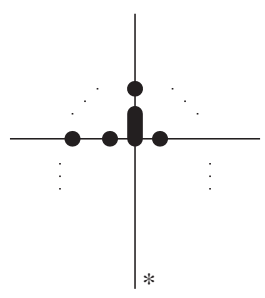

(a) Type I

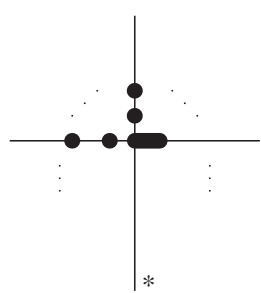

(b) Type II

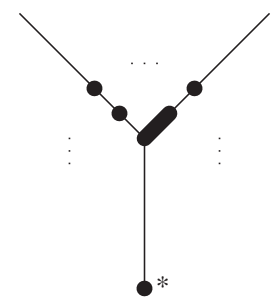

(c) Type III

Figure 6. The three types of exceptional critical 1-cells. For each type, all that is drawn are the relevant edges of the tree $T: T$ may have other essential vertices, and the essential vertex may have degree greater than 4 (for Types I and II) or 3 (for Type III), as suggested by the ellipses. As exceptional cells are critical, the diagrams depict reduced 1-cells, even though a subdivision of $T$ is not shown.

of necessary 1-cell, $d c$ also appears nontrivially in $A_{c_{1}, \ldots, c_{k}}(d f(a, \vec{x}))$. Thus, $M_{\omega}$ is a lower triangular matrix, all of whose diagonal entries are 1 . Therefore, $M_{\omega}$ is invertible.

At this point, we have associated to each necessary form $\omega$ an invertible matrix $M_{\omega}$. We now use these matrices associated to forms to define a matrix $M_{c}$ for each reduced 1-cell $c$.

Let $c$ be a reduced 1-cell.

If $c=(a, d, \vec{x})$ is not critical, then $c$ is necessary for the necessary 0 -form $\omega=$ $f(a, \vec{x})$ (Lemma 5.4). Define $M_{c}$ to be the matrix $M_{\omega}$, but with a 0 on the diagonal in the $r i(c)^{\text {th }}$ row. In terms of multiplication by $M_{c}$, this will correspond to replacing $d c$ with a cohomologically equivalent cochain determined by the coboundary of $\omega$.

If $c$ is critical, there are three types of exceptions we must make when defining $M_{c}$. Before stating the exceptions, we state the general cases. If $c$ is critical, not exceptional, and not necessary, define the matrix $M_{c}$ to be the identity matrix. If $c$ is critical, not exceptional, and necessary, define $M_{c}$ to be the matrix $M_{\omega}$ for any 1 -form $\omega$ for which $c$ is necessary. This corresponds to rewriting $d c$ with a 1-cochain that will eliminate the relation corresponding to $\omega$. We will prove momentarily that $M_{c}$ is well defined.

We now state the three types of exceptions. The motivation for the definitions of $M_{c}$ for the exceptional cases is not intuitively apparent, but the lemmas in the remainder of this section will justify our choices. All of the exceptional types have $n=5$. Let $c=(a, d, \vec{x})$ be critical. Assume that there exist two directions dir 1 and $\operatorname{dir} 2$ from $a$ for which $x_{\operatorname{dir} 1}, x_{\operatorname{dir} 2} \geq 2$. Without loss of generality, assume $\operatorname{dir} 1<\operatorname{dir} 2$. Let $\operatorname{dir} 3$ be: $\operatorname{dir} 1$ if $x_{\operatorname{dir} 1}=3$, or $\operatorname{dir} 2$ if $x_{\operatorname{dir} 2}=3$, or the unique index such that $x_{\mathrm{dir} 3}=1$. As $|\vec{x}|=5$, dir3 is uniquely determined. 
(1) (Type I) $0<\operatorname{dir} 1<\operatorname{dir} 2<\operatorname{dir} 3$, and $d=\operatorname{dir} 2: M_{c}$ is the identity matrix.

(2) (Type II) $0<\operatorname{dir} 1<\operatorname{dir} 2<\operatorname{dir} 3$, and $d=\operatorname{dir} 3$ : Let $c^{\prime}$ be the critical cell $(a, \operatorname{dir} 2, \vec{x})$ - that is, replace the edge of $e$ in direction $\operatorname{dir} 3$ from $a$ with an edge in direction $\operatorname{dir} 2$ from $a$. Then $M_{c}$ is the $r m \times r m$ identity matrix, but with an extra 1 in the $r i(c)^{\text {th }}$ row and $r i\left(c^{\prime}\right)^{\text {th }}$ column. Note as defined $c^{\prime}<_{r} c$, so $M_{c}$ is upper triangular. We want $M_{c}$ to be lower triangular, so we will slightly modify the order $<_{r}$ in Convention 5.8, below. The Type I cell $c^{\prime}$ and the Type II cell $c$ are said to correspond to each other. Note there is a bijection between cells of Type I and cells of Type II given by this correspondence.

(3) (Type III) $0=\operatorname{dir} 3<\operatorname{dir} 1<\operatorname{dir} 2$, and $d=\operatorname{dir} 2$ : For $i=1, \ldots, \operatorname{deg}(a)-1$, define $\vec{y}_{i}$ to be the $a$-vector which is $\vec{x}$, but with $\left(\vec{y}_{i}\right)_{0}=x_{0}-1=0$ and $\left(\vec{y}_{i}\right)_{i}=x_{i}+1$. Let $\vec{u}^{\prime} \in \mathbb{F}_{2}^{r m}$ be the vector whose only nonzero entries are exactly a 1 in the $r i\left(c^{\prime}\right)^{\text {th }}$ row for every reduced cell $c^{\prime}$ such that $c^{\prime}=c$ or $c^{\prime}=\left(a, \operatorname{dir} 2, \vec{y}_{i}\right)$ for some $i \in\{1, \ldots, \operatorname{deg}(a)-1\}, i \neq d$. Define $M_{c}$ to be the $r m \times r m$ identity matrix $I_{m}$, but with the $r i(c)^{\text {th }}$ row replaced by the transpose $\left(\vec{u}^{\prime}\right)^{\top}$. Note that $c<_{r}\left(a, \operatorname{dir} 2, \vec{y}_{i}\right)$ for each $i \in\{1, \ldots, \operatorname{deg}(a)-1\}$, so $M_{c}$ is lower triangular.

Convention 5.8 (A modification to $<_{r}$ ). We slightly modify the total order $<_{r}$ on reduced 1-cells by having corresponding exceptional critical cells of Types I and II switch places. By convention, the matrices $M_{\omega}$ and $M_{c}$ defined above are defined using this modified total order. All further references to $<_{r}$, and the associated functions $r i$ and $c i$, will be to the modified total order.

Note that with this new convention, Lemma 5.6 no longer holds for necessary forms $\omega$ where the associated necessary 1-cell is exceptional of Type I - in fact, instead of being lower triangular, $M_{\omega}$ will be upper triangular. This is fine, as $M_{\omega}$ was not used to define any matrix $M_{c}$. It is a small exercise to verify that, for all other cases, Lemma 5.6 still holds.

Lemma $5.9\left(M_{c}\right.$ is well defined). Let $c$ be a reduced 1-cell. The matrix $M_{c}$ is well defined and lower triangular.

Proof. For the three exceptional cases, the matrix $M_{c}$ was uniquely determined. If $c$ is exceptional of Type I, $M_{c}$ is the identity and is lower triangular. If $c$ is exceptional of Type II, then Convention 5.8 makes $M_{c}$ lower triangular. If $c$ is exceptional of Type III, the argument that $M_{c}$ is lower triangular given in the definition of $M_{c}$ still applies, even with Convention 5.8.

Aside from the three exceptional cases, if $c$ is not necessary or not critical, the matrix $M_{c}$ is uniquely specified and lower triangular by Corollary 5.7. So, assume $c$ is necessary and critical. Corollary 5.7 shows that $M_{c}$ will be lower triangular if it is well defined. To claim that $M_{c}$ is well defined is to claim that, if $\omega_{1}$ and $\omega_{2}$ are any two 1 -forms for which $c$ is necessary, $M_{\omega_{1}}=M_{\omega_{2}}$. Let $c=(a, d, \vec{x})$. By the definition 
of necessary, there exist critical 1-cells $c_{1}=\left(b_{1}, d_{1}, \vec{y}\right)$ and $c_{2}=\left(b_{2}, d_{2}, \vec{z}\right)$ such that $\omega=f(a, \vec{x}) \wedge d c_{1}$ and $\omega=f(a, \vec{x}) \wedge d c_{2}$. By the Upper Bound Lemma (Lemma 3.9), the directions $\alpha_{1}$ and $\alpha_{2}$ from $a$ to $b_{1}$ and $b_{2}$, respectively, are such that $\vec{x}_{\alpha_{1}}=n-y_{0} \geq 2$ and $\vec{x}_{\alpha_{2}}=n-z_{0} \geq 2$. By Lemma 5.5, $0<\alpha_{1}<d$ and $0<\alpha_{2}<d$, so if $\alpha_{1} \neq \alpha_{2}, c$ is an exceptional case of Type II. Since we are not addressing the exceptional cases, $\alpha_{1}=\alpha_{2}$.

The formulas $\vec{x}_{\alpha_{1}}=n-y_{0}$ and $\vec{x}_{\alpha_{2}}=n-z_{0}$ imply $y_{0}=z_{0}$. By the restricting the differential (Corollary 4.7), a nonzero term of $d f(a, \vec{x})$ is of the form $d s$, where $s$ is a reduced 1-cell which lies over the vertex $a$. Since $y_{0}=z_{0}$ and $a$ is in direction 0 from both of $b_{1}$ and $b_{2}$, by the Upper Bound Lemma (3.9), [s] has an upper bound with $\left[c_{1}\right]$ if and only if $[s]$ has an upper bound with $\left[c_{2}\right]$. In particular, by the definition of annihilator, $A_{c_{1}}(d s)=A_{c_{2}}(d s)$ for each such $s$-i.e. $A_{c_{1}}(d f(a, \vec{x}))=$ $A_{c_{2}}(d f(a, \vec{x}))$. The equality of the matrices $M_{\omega_{1}}$ and $M_{\omega_{2}}$ follows.

5.3. Changing bases and finishing Theorem 5.2. We now have lower triangular matrices $M_{c}$ for each reduced 1-cell $c$. We want to use the matrices $M_{c}$ to define a change of basis for $H^{1}\left(B_{n} T\right)$.

From now on, we think of the matrices $M_{c}$ as acting on reduced cocycles, where we identify a reduced cocycle $d c^{\prime}$ and the vector in $\mathbb{F}_{2}^{r m}$ whose only nonzero entry is in the $\operatorname{ri}\left(c^{\prime}\right)^{\text {th }}$ row.

To define our change of basis, we need to specify how to rewrite duals of critical cells. We do so on the cochain level, using 1 -forms associated to both critical and noncritical reduced 1-cells. For critical reduced 1-cells, we define a matrix $M s$. For noncritical reduced 1-cells, we define a matrix $M t$. We multiply the matrices $M s$ and $M t$ to define the desired change of basis matrix $M$, as follows.

Definition 5.10 (The matrices $M s, M t$, and $M$ ). Define the matrix $M s$ as:

$$
M s:=\prod_{\substack{i=1 \\ s i(c)=i}}^{s m} M_{c},
$$

where the product is over all critical cells $c$ and is written so that the $<_{r}$-largest cell $c$ is such that $M_{c}$ is on the right, applied first to any target vector. Define the matrix $M t$ as:

$$
M t:=\prod_{\substack{i=1 \\ t i(c)=t m-1+i}}^{t m} M_{c},
$$

where the product is over all non-critical reduced cells $c$ and is written so that the $<_{r}$-smallest cell $c$ is such that $M_{c}$ is on the right, applied first to any target vector. Define the matrix $M$, which will be the desired change of basis matrix, as:

$$
M:=M t M s .
$$


The matrices $M s, M t$, and $M$ have many nice properties, some of which we describe now.

Let $c_{0}$ be a reduced 1-cell. By definition, $M_{c} d c_{0}=d c_{0}$ for every $c \neq c_{0}$. Also by definition, $M_{c_{0}} d c_{0}$ consists of terms of the form $d c_{0}^{\prime}$, where $c_{0}$ and $c_{0}^{\prime}$ lie over the same vertex. Since each $M_{c}$ is lower triangular (Lemma 5.9), $c_{0} \leq_{r} c_{0}^{\prime}$, even when $c$ is exceptional.

If $c_{0}$ is a critical 1-cell, then $M t d c_{0}=d c_{0}$ and

$$
M s d c_{0}=\prod_{\substack{i=1 \\ s i(c)=i}}^{s m} M_{c} d c_{0}=M_{c_{0}} d c_{0} .
$$

This means that $M s$ agrees with the matrix $M_{c_{0}}$ in the $r i\left(c_{0}\right)^{\text {th }}$ column. Since $M_{c}$ is lower triangular and invertible for any critical 1-cell $c$, the matrix $M s$ is also lower triangular and invertible.

If $c_{0}$ is a noncritical 1 -cell, $M_{c_{0}} d c_{0}$ is by definition cohomologous to $d c_{0}$. As $M_{c_{0}}$ is the identity outside of column $r i\left(c_{0}\right)$, the matrix $M t$ preserves cohomology classes. As the matrix $M_{c}$ is lower triangular for each noncritical 1-cell $c$, the matrix $M t$ is lower triangular. Moreover, as the matrix $M_{c_{0}}$ contains a 0 on the diagonal in the $r i\left(c_{0}\right)^{\text {th }}$ place, the matrix $M t$ has no nonzero entries in the $r i\left(c_{0}\right)^{\text {th }}$ row. Finally, since $c_{0}$ is noncritical, $M s d c_{0}=d c_{0}$.

These observations imply that $M=M t M s$, restricted to basic 1-forms corresponding to critical 1-cells, is a change of basis matrix on cohomology classes, where for a critical 1-cell $c, M c^{*}=[M d c]$. We now have our change of basis matrix $M$ on $H^{1}\left(B_{n} T\right)$ and therefore on $H^{*}\left(B_{n} T\right)$. We may thus write $M$ as acting on cohomology classes (like $c^{*}$ ) instead of cochains (like $d c$ ), and will do so freely from now on. For future reference, we record some of our observations in a theorem:

Theorem 5.11 (Change of Basis Theorem).

(1) The matrix $M$ gives a change of basis isomorphism for $H^{1}\left(B_{n} T\right)$ and therefore $H^{*}\left(B_{n} T\right)$. For a critical $1-$ cell $c, M c^{*}:=[M d c]$.

(2) The matrices $M, M s$, and $M t$ are lower triangular. The matrix $M s$ is invertible.

(3) For a critical 1-cell c, Msdc= $M_{c} d c$. The cochain $M_{c} d c$ consists of terms of the form $d c_{0}^{\prime}$, where $c$ and $c_{0}^{\prime}$ lie over the same vertex and $c \leq_{r} c_{0}^{\prime}$.

(4) The matrix Mt preserves cohomology classes.

We need to analyze the effect of $M$ on all of cohomology, not just $H^{1}\left(B_{n} T\right)$. In particular, we need to know how $M$ affects cup products.

Lemma 5.12 (Cup products after changing basis). Let $c$ and $c^{\prime}$ be critical 1-cells, where $c \leq_{r} c^{\prime}$. If $M c^{*} \cup M\left(c^{\prime}\right)^{*} \neq[0]$, then $[c]$ and $\left[c^{\prime}\right]$ have a least upper bound $[s]$ with reduced representative $s$ and either: 
(1) $s$ is critical, or

(2) $s$ is not critical and $c$ is exceptional of Type I.

Proof. We will repeatedly use the properties of $M, M s$, and $M t$ stated in Theorem 5.11 to prove this lemma, and do so without further reference.

Since $M=M t M s$ and $M t$ preserves cohomology classes, $M c^{*} \cup M\left(c^{\prime}\right)^{*} \neq[0]$ if and only if $[M s d c] \cup\left[M s d c^{\prime}\right] \neq[0]$. We have that $M s d c=M_{c} d c$ and $M s d c^{\prime}=$ $M_{c^{\prime}} d c^{\prime}$. Thus, $M c^{*} \cup M\left(c^{\prime}\right)^{*} \neq[0]$ if and only if $\left[M_{c} d c\right] \cup\left[M_{c^{\prime}} d c^{\prime}\right] \neq[0]$.

Express $c$ as $(a, e, \vec{x})$ and $c^{\prime}$ as $\left(a^{\prime}, e^{\prime}, \vec{x}^{\prime}\right)$.

If $a=a^{\prime}$, then every summand in both $M_{c} d c$ and $M_{c^{\prime}} d c^{\prime}$ will lie over $a$. By the Upper Bound Lemma (Lemma 3.9), no two reduced 1-cells which lie over the same vertex have an upper bound. By the definition of $\wedge$, it follows that $M_{c} d c \wedge M_{c^{\prime}} d c^{\prime}$ is the 0 function, so $[M s d c] \cup\left[M s d c^{\prime}\right]=[0]$. Thus, we may assume that $a \neq a^{\prime}$.

Consider summands $d c_{0}$ and $d c_{0}^{\prime}$ of $M_{c} d c$ and $M_{c^{\prime}} d c^{\prime}$, respectively. Express $c_{0}$ as $\left(a, d_{0}, \vec{y}\right)$ and $c_{0}^{\prime}$ as $\left(a^{\prime}, d_{0}^{\prime}, \vec{y}^{\prime}\right)$. By the definition of $M_{c}$ and restricting the differential (Corollary 4.7), $\vec{y}$ differs from $\vec{x}$ for at most two indices, namely 0 and some index $\operatorname{dir}_{0}$. If $\vec{y} \neq \vec{x}$ then $y_{0}=x_{0}-1$ and $y_{\operatorname{dir}_{0}}=x_{\operatorname{dir}_{0}}+1$. In particular, $x_{0} \geq y_{0}$. Furthermore, by definition, if $c$ is not exceptional of Type III, then $d_{0}^{\prime}=d_{0}$. Similar statements hold for $d c_{0}^{\prime}$, but we will only need to use that $x_{0}^{\prime} \geq y_{0}^{\prime}$.

We claim that $\left[M_{c} d c\right] \cup\left[M_{c^{\prime}} d c^{\prime}\right] \neq[0]$ if and only if $\left[M_{c} d c\right] \cup\left[d c^{\prime}\right] \neq[0]$, as follows.

Consider if $\left[M_{c} d c\right] \cup\left[d c^{\prime}\right]=[0]$ but $\left[M_{c} d c\right] \cup\left[M_{c^{\prime}} d c^{\prime}\right] \neq[0]$. Then there exists some summand $d c_{0}^{\prime} \neq d c^{\prime}$ of $M_{c^{\prime}} d c^{\prime}$ such that $\left[M_{c} d c\right] \cup\left[d c_{0}^{\prime}\right] \neq[0]$. Thus, there must exist a summand $d c_{0}$ of $M_{c} d c$ such that $d c_{0} \wedge d c_{0}^{\prime}$ is not cohomologous to 0 . In particular, $\left[c_{0}\right]$ and $\left[c_{0}^{\prime}\right]$ have an upper bound. By the Upper Bound Lemma, since $x_{0}^{\prime} \geq y_{0}^{\prime},\left[c_{0}\right]$ and $\left[c^{\prime}\right]$ have an upper bound. Since $\left[M_{c} d c\right] \cup\left[d c^{\prime}\right]=[0]$, there exists a sequence of Tietze transformations which equates $d c_{0} \wedge d c^{\prime}$ with $\left(M_{c} d c \wedge\right.$ $\left.d c^{\prime}-d c_{0} \wedge d c^{\prime}\right)$. Each Tietze transformation involving a relation will involve the coboundary of a necessary 1-form. By restricting the differential, such a 1-form has either the form $f(a, \vec{z}) d c^{\prime}$ or $f\left(a^{\prime}, \overrightarrow{z^{\prime}}\right) d c_{0}$ for some $a$-vector $\vec{z}$ or $a^{\prime}$-vector $\vec{z}^{\prime}$. By Lemma 5.5 , such a 1 -form must be of the form $f(a, \vec{z}) d c^{\prime}$, since $a$ is in direction 0 from $a^{\prime}$. By the definition of necessary, $f(a, \vec{z}) d c_{0}^{\prime}$ will also be a necessary 1 -form. It follows that a corresponding sequence of Tietze transformations equates $d c_{0} \wedge d c_{0}^{\prime}$ with $\left(M_{c} d c \wedge d c_{0}^{\prime}-d c_{0} \wedge d c_{0}^{\prime}\right)$. But then $\left[M_{c} d c \wedge d c_{0}^{\prime}\right]=[0]$, a contradiction.

To finish the claim, it remains to prove that if $\left[M_{c} d c\right] \cup\left[d c^{\prime}\right] \neq[0]$, then $\left[M_{c} d c\right] \cup$ $\left[M_{c^{\prime}} d c^{\prime}\right] \neq[0]$. If $\left[M_{c} d c\right] \cup\left[d c^{\prime}\right] \neq[0]$ but $\left[M_{c} d c\right] \cup\left[M_{c^{\prime}} d c^{\prime}\right]=[0]$, then there exists some summand - say $d c_{0}^{\prime}$ - of $M_{c^{\prime}} d c^{\prime}$ such that $d c_{0} \wedge d c^{\prime}$ and $d c_{0} \wedge d c_{0}^{\prime}$ are both in a single relation - that is, in the differential of a single necessary 1-form $\omega^{\prime}$. By restricting the differential, it must be that $\omega^{\prime}=f\left(a^{\prime}, \vec{z}\right) d c_{0}$ for some $a^{\prime}$-vector $\vec{z}$. This contradicts Lemma 5.5, as the direction from $a^{\prime}$ to $a$ is 0 . This proves the claim.

To prove the lemma, we show that if $\left[M_{c} d c\right] \cup\left[d c^{\prime}\right] \neq[0]$, then $[c]$ and $\left[c^{\prime}\right]$ have a least upper bound $[s]$ with reduced representative $s$, and either $s$ is critical or $s$ is 
not critical but $c$ is exceptional of Type I.

By Theorem 3.6, $\left[d c_{0}\right] \cup\left[d c_{0}^{\prime}\right] \neq[0]$ only if $\left[c_{0}\right]$ and $\left[c_{0}^{\prime}\right]$ have an upper bound. Let $\alpha>0$ be the direction from $a$ to $a^{\prime}$. By the Upper Bound Lemma, $\left[c_{0}\right]$ and $\left[c_{0}^{\prime}\right]$ have a common upper bound if and only if

$$
y_{\alpha}+y_{0}^{\prime} \geq n+\epsilon_{0},
$$

where $\epsilon_{0}:=\epsilon\left(c_{0}, \alpha\right)$ is the upper bound constant. We record the interpretation of equation 1 for the case that $c_{0}=c$, as these two equations are the heart of what remains in the proof. We have that $[c]$ and $\left[c^{\prime}\right]$ have a common upper bound if and only if

$$
x_{\alpha}+x_{0}^{\prime} \geq n+\epsilon,
$$

where $\epsilon:=\epsilon(c, \alpha)$.

If $[c]$ and $\left[c^{\prime}\right]$ have a common upper bound, by the Upper Bound Lemma, then $x_{\alpha} \geq 2$. Also, by definition, $x_{d} \geq 1$.

Consider the number $y_{\alpha}-\epsilon_{0}$. By definition, $\vec{y}$ differs from $\vec{x}$ only in the $0^{\text {th }}$ and $\left(d_{0}^{\prime}\right)^{\text {th }}$ entries. Since $\alpha>0, y_{\alpha}=x_{\alpha}$ unless $\alpha=d_{0}^{\prime}$. But unless $c$ is exceptional of Type III, $d_{0}^{\prime}=d_{0}$, and when $\alpha=d_{0}^{\prime}, \epsilon_{0}=1$. Therefore, unless $\alpha=d_{0}^{\prime}$ and $c$ is exceptional of Type III, $y_{\alpha}-\epsilon_{0}=x_{\alpha}$. Unless $\alpha=d, \epsilon=0$, so $x_{\alpha}=x_{\alpha}-\epsilon$. Thus, unless $\alpha=d$, or $\alpha=d_{0}^{\prime}$ and $c$ is exceptional of Type III, if $\left[c_{0}\right]$ and $\left[c^{\prime}\right]$ then $[c]$ and $\left[c^{\prime}\right]$ have a common upper bound $[s]$.

Consider the case that $c$ is exceptional of Type III. Let $\omega=f(a, \vec{x}) d c_{1}$ be a necessary 1-form for which $c$ is necessary. Let $\alpha_{1}$ be the direction from $a$ towards the vertex over which $c_{1}$ lies. By the Upper Bound Lemma, if $\left[c_{0}\right]$ and $\left[c^{\prime}\right]$ have an upper bound, then $y_{\alpha}-\epsilon_{0} \geq 2$. By the definition of $M_{c}$, if $\alpha \neq \alpha_{1}$, then $y_{\alpha}-\epsilon_{0} \leq 1$. Thus, if $\left[c_{0}\right]$ and $\left[c^{\prime}\right]$ have an upper bound, then $\alpha=\alpha_{1}$. If $[c]$ and $\left[c^{\prime}\right]$ also have an upper bound, then $x_{0}^{\prime}=3$. By definition, any term $d c_{0}^{\prime}$ of $M_{c} d c$ is such that $\left[c_{0}^{\prime}\right]$ and $\left[c_{1}\right]$ have an upper bound. By the Upper Bound Lemma, we also have that $\left[c_{0}^{\prime}\right]$ and $\left[c^{\prime}\right]$ have an upper bound. Moreover, consider the 1-form $\omega^{\prime}=f(a, \vec{x}) d c^{\prime}$. By restricting the differential (Corollary 4.7), we have that $d \omega^{\prime}=M_{c} d c \wedge d c^{\prime}$. Thus, $\left[M_{j} d c\right] \cup\left[M_{j} d c^{\prime}\right]=[0]$. If $[c]$ and $\left[c^{\prime}\right]$ do not also have an upper bound, then $x_{0}^{\prime}=2$ and $y_{\alpha}=3$. Thus there is only one such $c_{0}$ : when $d_{0}^{\prime}=\alpha$. By restricting the differential, we have that $d\left(f(a, \vec{y}) d c^{\prime}\right)=d c_{0} \wedge d c^{\prime}$, so

$$
\left[M_{j} d c\right] \cup\left[M_{j} d c^{\prime}\right]=\left[M_{c} d c \wedge d c^{\prime}\right]=\left[d c_{0} \wedge d c^{\prime}\right]=[0] .
$$

Now consider the case when $c$ is not exceptional of Type III. If $\alpha=d$, we have a number of subcases.

- If $c$ is exceptional of Type II, then $y_{d}=1$. This implies $x_{d}^{\prime} \geq n-1$, so $\left[c_{0}\right]$ and $\left[c^{\prime}\right]$ cannot have an upper bound.

- If $c$ is not necessary and $c$ is not exceptional of Type II, then $M_{c}$ is the identity, and $c_{0}=c$. If $\left[c_{0}\right]$ and $\left[c^{\prime}\right]$ have an upper bound $[s]$, then $[c]=\left[c_{0}\right]$ and $\left[c^{\prime}\right]$ have the upper bound $[s]$. Let $s$ be the reduced representative of $[s]$. If $e$ is not 
disrespectful in $s, c$ would be necessary for the necessary 1-form $f(a, \vec{x}) d c^{\prime}$, contradicting that $c$ is not necessary. Thus, if $\left[c_{0}\right]$ and $\left[c^{\prime}\right]$ have an upper bound, $e$ must be disrespectful in $s$, and by the Upper Bound Lemma, $s$ is critical.

- If $c$ is necessary and not exceptional of Type II, then let $\omega=f(a, \vec{x}) d c_{1}$ be a 1-form for which $c$ is necessary. Let $\alpha_{1}$ be the direction from $a$ towards the vertex over which $c_{1}$ lies. By Lemma 5.5, $0<\alpha_{1}<d$, and $x_{\alpha_{1}} \geq 2$. Since $c$ is not exceptional of Type III, $d_{0}^{\prime}=d_{0}$. By equations 1 and $2, y_{\alpha}-\epsilon_{0}=x_{\alpha}$. Thus, since $x_{\alpha}^{\prime} \leq n-2$, if $\left[c_{0}\right]$ and $\left[c^{\prime}\right]$ have an upper bound then $x_{\alpha} \geq 2+\epsilon_{0}$.

- If $n=4$, since $|\vec{x}|=n, x_{\alpha}=x_{\alpha_{1}}=2$ and $c$ is completely determined. In this case, by restricting the differential, we have $A_{c_{1}}(d f(a, \vec{x}))=d c$, so $c_{0}=c$. But then $\epsilon_{0}=1$, and $2=x_{\alpha} \geq 2+1=3$. Thus, if $n=4$, then $\left[c_{0}\right]$ and $\left[c^{\prime}\right]$ cannot have an upper bound.

- If $n=5$, since $|\vec{x}|=5$ we know there is one vertex of $c$ unaccounted for: there is some index $\beta$ such that $x_{\beta}=1$ when $\beta \notin\left\{\alpha, \alpha_{1}\right\}$ or $x_{\beta}=3$ when $\beta \in\left\{\alpha, \alpha_{1}\right\}$. Since $c$ is not exceptional of Type III, $\beta \neq 0$.

* If $\beta>d$, then $c$ is of Type $\mathrm{I}$, and so $c_{0}=c$ by the definition of $M_{c}$ and the lemma holds.

$*$ If $\beta=d$, then the only nonzero entries of $\vec{x}$ are $x_{\alpha_{1}}=2$ and $x_{d}=3$. Since $\alpha \neq \alpha_{1}$ and $x_{\alpha} \geq 2+\epsilon_{0}, \alpha=d$. By restricting the differential we have that $d \omega=d c \wedge d c_{1}$, so $M_{c} d c=d c$ and $c_{0}=c$. Since $c^{\prime}$ lies over a vertex in direction $\alpha=d$ from $a$, the lemma then follows from the Upper Bound Lemma.

* If $\beta<d$, then $c$ is not necessary: the cell $(a, \beta, \vec{x})$ is the necessary 1-cell for $f(a, \vec{x}) d c_{1}$. This contradicts the assumption that $c$ is necessary, so the lemma holds.

It remains to prove the lemma when $c$ is not exceptional of Type III and $\alpha \neq d$. In this case, by equations (1) and (2), if $\left[c_{0}\right]$ and $\left[c^{\prime}\right]$ have a common upper bound then $[c]$ and $\left[c^{\prime}\right]$ have a common upper bound $[s]$. Let $s$ be the reduced representative of $[s]$. If $e$ is disrespectful in $s$, we're done. Note that if $c$ is exceptional of Type II, then $e$ is disrespectful in $s$, by the Upper Bound Lemma. If $e$ is respectful in $s$, then by the Upper Bound Lemma, $0<\alpha<d, x_{\alpha}+y_{0}=n$, and $x_{i}=0$ for all $0<i<d$, $i \neq \alpha$. The lemma allows for $c$ to be exceptional of Type I in this case. Consider if $c$ is not exceptional (as we have addressed each of the three exceptional cases). By definition, any term $d c_{0}^{\prime}$ of $M_{c} d c$ is such that $\left[c_{0}^{\prime}\right]$ and $\left[c_{1}\right]$ have an upper bound. By the Upper Bound Lemma, we also have that $\left[c_{0}^{\prime}\right]$ and $\left[c^{\prime}\right]$ have an upper bound. Moreover, consider the 1-form $\omega^{\prime}=f(a, \vec{x}) d c^{\prime}$. By restricting the differential, we have that $d \omega^{\prime}=M_{c} d c \wedge d c^{\prime}$. Thus, $\left[M_{j} d c\right] \cup\left[M_{j} d c^{\prime}\right]=[0]$. This finishes the proof.

Lemma 5.12 tells us that multiplication by $M$ is very nice in ensuring that when $M c^{*}$ and $M\left(c^{\prime}\right)^{*}$ cup nontrivially, $[c]$ and $\left[c^{\prime}\right]$ have an upper bound with critical 
representative, with only one type of exception. If $c$ is exceptional of Type I, then Lemma 5.12 does not say much about $M c^{*}$. There is something to say, though:

Lemma 5.13. Let $c_{1}=\left(a, d_{1}, \vec{x}\right)$ be a critical cell of Type $\mathrm{I}$ and let $c_{2}=\left(a, d_{2}, \vec{x}\right)$ be the corresponding critical cell of Type II. Let $d_{0}$ be the index of the remaining nonzero coordinate of $\vec{x}$. For any critical 1 -cell $c^{\prime}=\left(a^{\prime}, e^{\prime}, \vec{x}^{\prime}\right)$,

(1) if $M c_{1}^{*} \cup M\left(c^{\prime}\right)^{*} \neq[0]$ then $\left[c_{2}\right]$ and $\left[c^{\prime}\right]$ have a least upper bound $[s], c_{2}^{*} \cup$ $\left(c^{\prime}\right)^{*} \neq[0]$, and $a^{\prime}$ is in direction $d_{0}$ from $a$.

(2) if $M c_{2}^{*} \cup M\left(c^{\prime}\right)^{*} \neq[0]$ then $\left[c_{2}\right]$ and $\left[c^{\prime}\right]$ have a least upper bound $[s], c_{2}^{*} \cup$ $\left(c^{\prime}\right)^{*} \neq[0]$ and $a^{\prime}$ is in direction $d_{1}$ from $a$.

The way to think of this is that $M c_{1}^{*}$ is the $d_{0}$ 'portion' of $c_{2}^{*}$ (and $c_{1}^{*}$ ), while $M c_{2}^{*}$ is the $d_{1}$ 'portion' of $c_{2}^{*}$ (and trivially $c_{1}^{*}$ ).

Proof. By the Upper Bound Lemma (Lemma 3.9), $\left[c_{1}\right]$ and $\left[c^{\prime}\right]$ have an upper bound if and only if $a^{\prime}$ is in direction $d_{0}$ from $a$ and $\left[c_{2}\right]$ and $\left[c^{\prime}\right]$ have an upper bound. Also, that $\left[c_{2}\right]$ and $\left[c^{\prime}\right]$ have an upper bound implies $a^{\prime}$ is in either of the directions $d_{0}$ or $d_{1}$ from $a$. By the Upper Bound Lemma, if $\left[c_{2}\right]$ and $\left[c^{\prime}\right]$ have an upper bound $[s]$, then the reduced representative $s$ of $[s]$ is critical, so $c_{2}^{*} \cup\left[d c^{\prime}\right] \neq[0]$.

If $M c_{1}^{*} \cup M\left[d c^{\prime}\right] \neq[0]$, then by Lemma 5.12, $\left[c_{1}\right]$ and $\left[c^{\prime}\right]$ have an upper bound. Thus, by above, if $M c_{1}^{*} \cup M\left[d c^{\prime}\right] \neq[0]$, then $c_{2}^{*} \cup\left[d c^{\prime}\right] \neq[0]$.

If $M c_{2}^{*} \cup M\left[d c^{\prime}\right] \neq[0]$, then by Lemma $5.12,\left[c_{2}\right]$ and $\left[c^{\prime}\right]$ have an upper bound, so by above, $c_{2}^{*} \cup\left[d c^{\prime}\right]=[d s] \neq[0]$. By above, $a^{\prime}$ is in one of the directions $d_{0}$ or $d_{1}$ from $a$. We prove, assuming $M c_{2}^{*} \cup M\left[d c^{\prime}\right] \neq[0]$, that $a^{\prime}$ cannot be in direction $d_{0}$ from $a$. By the definition of $M$ and the matrices $M_{c_{1}}$ and $M_{c_{2}}, M d c_{2}=d c_{1}+d c_{2}$. So, $M d c_{2} \wedge d c^{\prime}=d c_{1} \wedge d c^{\prime}+d c_{2} \wedge d c^{\prime}$. But this is precisely the coboundary of the 1 -form $f(a, \vec{x}) d c^{\prime}$ by restricting the differential (Corollary 4.7). Thus,

$$
[0]=\left[M d c_{2} \wedge d c^{\prime}\right]=\left[M d c_{2}\right] \cup\left[M d c^{\prime}\right]=M c_{2}^{*} \cup M\left[d c^{\prime}\right] .
$$

This contradicts the assumption that $M c_{2}^{*} \cup M\left[d c^{\prime}\right] \neq[0]$. We have proven that if $M c_{2}^{*} \cup M\left[d c^{\prime}\right] \neq[0]$ then $a^{\prime}$ is in direction $d_{1}$ from $a$. This finishes the proof.

We are now ready to state and prove the final step of Theorem 5.2.

Definition 5.14 (The simplicial complex $\Delta$ ). Define the finite simplicial complex $\Delta$ as follows. The vertex set of $\Delta$ is identified with the set $\left\{M c^{*} \mid c\right.$ a critical 1-cell $\}$. A vertex $M c^{*}$ is said to lie over the vertex of $T$ over which $c$ lies. A set of vertices $\left\{M c_{1}^{*}, M c_{2}^{*}\right\}$ span a 1-simplex, labelled $M c_{1}^{*} \cup M c_{2}^{*}$, if and only if $M c_{1}^{*} \cup M c_{2}^{*}$ is nontrivial.

Theorem 5.15 (The exterior face algebra structure). We have that

$$
H^{*}\left(B_{n} T\right) \cong \Lambda(\Delta)
$$


Proof. Consider the map $\Psi: \Lambda(\Delta) \rightarrow H^{*}\left(B_{n} T\right)$ given by, for $c$ a critical 1-cell, mapping the element in $\Lambda(\Delta)$ corresponding to the 0-cell of $\Delta$ labelled $M c^{*}$ to the 1-cohomology class $M c^{*}$. Since critical 1-cells form a free basis for $H^{1}\left(B_{n} T\right)$ and $M$ is a change of basis matrix, the map extends to all of $H^{1}\left(B_{n} T\right)$. By the definition of $\Delta, \Psi$ is surjective onto $H^{1}\left(B_{n} T\right)$ and moreover extends linearly to a surjective homomorphism, since $H^{1}\left(B_{n} T\right)$ generates all of $H^{*}\left(B_{n} T\right)$ (see Theorem 3.6).

We claim that $\Psi$ is also injective. Since $n \in\{4,5\}$, it suffices to consider 1-cells of $\Delta$. A simple counting argument will finish the result. For any 1-cell $\left\{M c_{1}^{*}, M c_{2}^{*}\right\}$ of $\Delta$, by Lemma 5.12 there is a critical 2-cell $s$ such that $[s]$ is the least upper bound of [ $\left.c_{1}\right]$ and $\left[c_{2}\right]$, unless possibly one of $c_{1}$ or $c_{2}$ is exceptional of type I. By Theorem 3.6, $s$ uniquely determines and is uniquely determined by $c_{1}$ and $c_{2}$. By Lemma 5.13, even when one of $c_{1}$ or $c_{2}$ is exceptional of Type I, there is still a uniquely determined critical 2-cell $s$ corresponding to $c_{1}$ and $c_{2}$. Thus, there are at most as many edges of $\Delta$ as there are critical 2-cells. Since $\Psi$ is surjective and by Theorem 3.6, $H^{2}\left(B_{n} T\right)$ has rank equal to the number of critical 2-cells, $\Psi$ must also be injective. Thus, $\Psi$ is the desired isomorphism.

Theorem 5.15 gives us an important strengthening of Lemmas 5.12 and 5.13:

Corollary 5.16 (Multiplication by $M)$. Let $c=(a, d, \vec{x})$ and $c^{\prime}=\left(a^{\prime}, d^{\prime}, \vec{x}^{\prime}\right)$ be critical 1-cells with $c \leq_{r} c^{\prime}$. Then

$$
M c^{*} \cup M\left(c^{\prime}\right)^{*} \neq[0]
$$

if and only if $[c]$ and $\left[c^{\prime}\right]$ have a least upper bound $[s]$ with reduced representative s and either

(1) $c$ is not exceptional of Types I or II and s is critical,

(2) $c$ is exceptional of Type I and s is not critical, or

(3) $c$ is exceptional of Type II, $s$ is critical, and the direction $\alpha$ from a to $a^{\prime}$ is not the smallest direction for which $x_{\alpha} \neq 0$.

The results of this section show that exterior face algebra structures do crop up for cohomology rings of tree braid groups, but restrictive assumptions were made on the tree braid groups in question. We have shown that if $n<6$ or $T$ is linear, then $H^{*}\left(B_{n} T\right)$ is an exterior face algebra. We conjecture the converse holds:

Conjecture 5.17 (Arbitrary number of strands). Let $T$ be a tree. Then $H^{*}\left(B_{n} T\right)$ is an exterior face algebra if and only if $n<6$ or $T$ is linear.

Our conjecture is based on the reasoning that, for $T$ a nonlinear tree and $n \geq 6$, we may find a particular structure in $H^{*}\left(B_{n} T\right)$ which we believe prevents $H^{*}\left(B_{n} T\right)$ from being an exterior face algebra. We do not go into the details here, but we claim if we apply the change of basis techniques of this section to $H^{*}\left(B_{n} T\right)$ for $n \geq 6$, 
the result is that $H^{*}\left(B_{n} T\right)$ is isomorphic to some particular quotient of an exterior face algebra. That quotient has as a subalgebra the quotient pictured in Figure 7. We believe that this subalgebra is 'poisonous': any algebra containing the given subalgebra in a 'nice' way will not be an exterior face algebra. Of course, we do not know the appropriate definition of 'nice', and showing that an algebra is not an exterior face algebra is very difficult, even in this restricted setting.

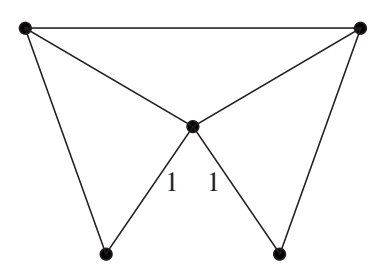

Figure 7. Shown is a simplicial complex. The 1s labelling two 1-cells of the simplicial complex indicate that in the corresponding exterior face algebra, we quotient by identifying the two edges. We believe that, if this quotient is a subalgebra of a given algebra in a nice way, then the given algebra cannot be an exterior face algebra. We also believe that the cohomology algebra of tree braid groups for nonlinear trees and at least 6 strands contain this quotient as a subalgebra in a nice way.

\section{Rigidity}

In this section, our goal is to prove Theorem 6.9, the rigidity of 4 and 5 strand tree braid groups. We do so by explicitly reconstructing the tree $T$ from the corresponding tree braid group $B_{n} T$. The tree $T_{\Delta}$, defined in the first subsection, will be the reconstruction desired, as proven in the second subsection.

6.1. The tree $\boldsymbol{T}_{\boldsymbol{\Delta}}$. In this subsection we try to construct a tree $T_{\Delta}$ from an arbitrary 1-dimensional simplicial complex $\Delta$. The tree $T_{\Delta}$ will be used in Section 6.2 to prove Theorem 6.9. In Theorem 6.9, we will show that, if $\Delta$ is the simplicial complex giving the exterior face structure for $H^{*}\left(B_{n} T\right)$ for some tree $T$ and $n=4$ or 5 , then $T_{\Delta}$ is homeomorphic to $T$. By Theorem 5.2, for $n=4$ or 5 there exists a unique 1dimensional simplicial complex associated to $H^{*}\left(B_{n} T\right)$ - this is why we restrict $\Delta$ to be 1-dimensional. Even with the dimension restriction, for many $\Delta, T_{\Delta}$ will not exist; it will turn out that $T_{\Delta}$ will exist exactly when we want it to (see Corollary 6.13).

Throughout this section, refer to Example 6.1 on page 505 for an example of the concepts defined. Also see Example 6.11 on page 513 for further reference.

Let $\Delta$ be an arbitrary 1-dimensional simplicial complex, and fix a constant $n \in$ $\{4,5\}$. For each vertex $v \in \Delta$, let $N_{v}$ denote the vertex neighborhood of $v$ in $\Delta$ : the set of all vertices $v^{\prime} \in \Delta$ adjacent to $v$. Let $\leq_{N}$ denote the preorder on vertices of 
$\Delta$ induced by vertex neighborhood inclusions, so $v \leq_{N} v^{\prime}$ if and only if $N_{v} \subseteq N_{v^{\prime}}$. Most pairs of vertices will not be comparable, but enough will be.

The preorder $\leq_{N}$ induces an equivalence relation $=_{N}$ on vertices of $\Delta$ : if $v \leq_{N} v^{\prime}$ and $v^{\prime} \leq_{N} v$, we write $v={ }_{N} v^{\prime}$. Note that $\leq_{N}$ induces a partial order on the vertex set $V(\Delta)$ of $\Delta$ modulo equivalence classes under $={ }_{N}$. We write the $\left(={ }_{N}\right)$-equivalence class of $v \in V(\Delta)$ as $[v]$. For an $\left(={ }_{N}\right)$-equivalence class $[v]$, the descendants of $[v]$ are those $\left(={ }_{N}\right)$-equivalence classes $\left[v^{\prime}\right]$ such that $\left[v^{\prime}\right] \leq[v]$ (i.e. $N_{v^{\prime}} \subseteq N_{v}$ ) and $N_{v^{\prime}} \neq \emptyset$. A descendant $\left[v^{\prime}\right]$ of $[v]$ is a child of $[v]$ and $[v]$ is a parent of $\left[v^{\prime}\right]$ if there are no descendants of $[v]$ between $\left[v^{\prime}\right]$ and $[v]$ with respect to $\leq_{N}$ - i.e. there does not exist a $w$ such that $N_{v^{\prime}} \subsetneq N_{w} \subsetneq N_{v}$.

We now define a tree $T_{\Delta}$ from the simplicial complex $\Delta$. Let $|\Delta|$ denote the number of vertices of $\Delta$, and for any subset $S$ of $V(\Delta)$, let $|S|$ denote its size.

Recall the function $Y_{m}(x)$, defined in Theorem 3.4. We will use $Y_{m}(x)$ repeatedly to define $T_{\Delta}$.

Let $v_{0}$ be a vertex of $\Delta$ which is maximal under $\leq_{N}$. Thus, $N_{v_{0}}$ is maximal under inclusion among all vertex neighborhoods of vertices of $\Delta$.

If $N_{v_{0}}$ is empty, define the tree $T_{\Delta}$ to be a radial tree (see Theorem 3.4) whose essential vertex $a$ has degree $\operatorname{deg}(a)$ such that

$$
Y_{n}(\operatorname{deg}(a))=|\Delta|
$$

if such a number exists. If such a number does not exist, then $T_{\Delta}$ is undefined.

Now assume that $N_{v_{0}}$ is nonempty. Define the finite undirected graph $H^{\prime}$ to be the neighborhood hierarchy of $\left[v_{0}\right]$, as follows. Create a vertex $p_{1}$ in $H^{\prime}$, and for each descendant $[v]$ of $\left[v_{0}\right]$ create a vertex $p_{[v]}$ in $H^{\prime}$, so that the vertex set of $H^{\prime}$ is

$$
\left\{p_{1}\right\} \cup\left\{p_{[v]} \mid[v] \text { is a descendant of }\left[v_{0}\right]\right\} \text {. }
$$

Every vertex $p_{[v]}$ is connected to $p_{\left[v^{\prime}\right]}$ for each child $\left[v^{\prime}\right]$ of $[v]$. Also, there is one edge connecting $p_{1}$ to $p_{\left[v_{0}\right]}$ (when $\Delta$ is as in Theorem 5.15, think of $p_{1}$ as corresponding to the cochain 1 , so that every 1-cochain is in its 'neighborhood').

If $n=4$, the neighborhood hierarchy $H^{\prime}$ is already almost the tree $T_{\Delta}$, but if $n=5$, the $H^{\prime}$ contains too many vertices. To address this issue, we define a subgraph $H$ of the graph $H^{\prime}$. If $n=4$, define the finite undirected graph $H$ to be $H^{\prime}$. If $n=5$, consider if there exists a child $\left[v_{0}^{\prime}\right]$ of $\left[v_{0}\right]$ such that

(1) $\left[v_{0}^{\prime}\right]$ has exactly half as many descendants as $\left[v_{0}\right]$, and

(2) if $[v]$ and $\left[v^{\prime}\right]$ are children of $\left[v_{0}\right]$ with a common descendant, then one of $[v]$ or $\left[v^{\prime}\right]$ is a descendant of $\left[v_{0}^{\prime}\right]$.

If such a child [ $\left.v_{0}^{\prime}\right]$ exists, define $H$ to be $H^{\prime}$ but with the descendants of $\left[v_{0}^{\prime}\right]$ removed from $H^{\prime}$ - that is, $H$ is $H^{\prime}$ minus the vertices corresponding to descendants of $\left[v_{0}^{\prime}\right]$ and any edges connecting them to any other vertices. If no such child exists, $H$ is undefined.

We want to construct a tree $T_{\Delta}$ by 'growing' $T_{\Delta}$ from the graph $H$. To do so, we want to associate to each vertex $p_{[v]}$ of $H$ a number $p \operatorname{deg}_{[v]}$, which will be the 
desired degree of $p_{[v]}$. We say $p_{[v]}$ is a leaf of $H$ if $[v]$ has no children. If $p_{[v]}$ is a leaf of $H$, then define $p \operatorname{deg}_{[v]}$ to be the positive integer greater than 2 satisfying

$$
Y_{(n-2)}\left(p \operatorname{deg}_{[v]}\right)=\left|N_{v}\right|
$$

for any $v \in[v]$; if such a number does not exist, $p \operatorname{deg}_{[v]}$ is undefined. If $p_{[v]}$ is not a leaf of $H$, then define $p \operatorname{deg}_{[v]}$ to be the positive integer greater than 2 satisfying

$$
Y_{2}\left(p \operatorname{deg}_{[v]}\right)=\left|\left[v^{\prime}\right]\right|
$$

for every child $\left[v^{\prime}\right]$ of $[v]$; if such a number does not exist, $p \operatorname{deg}_{[v]}$ is undefined. Also define $p \operatorname{deg}_{1}$ to be the positive integer greater than 2 satisfying

$$
Y_{2}\left(p \operatorname{deg}_{1}\right)=\left|\left[v_{0}\right]\right| ;
$$

if such a number does not exist, $p \operatorname{deg}_{1}$ is undefined.

If $p \operatorname{deg}_{[v]}$ exists, it is unique, since $Y_{2}$ and $Y_{(n-2)}$ are monotonic increasing on values greater than 2 .

We may now define the $T_{\Delta}$. The tree $T_{\Delta}$ is undefined if any of the following hold:

(1) $H$ is undefined;

(2) $H$ is not a tree;

(3) there exists $p_{[v]}$ in $H$ such that $p \operatorname{deg}_{[v]}$ is undefined, or $p \operatorname{deg}_{1}$ is undefined;

(4) there exists $p_{[v]}$ in $H$ which is not a leaf and [v] has more than $d_{[v]}-1$ children.

If we are not in one of the cases where $T_{\Delta}$ is undefined, then $T_{\Delta}$ is the tree $H$ together with some edges added for the purpose of increasing the degrees of the vertices $p_{[v]}$. Each distinct added edge connects a vertex $p_{[v]}$ to a distinct vertex of degree 1 in $T_{\Delta}$. We add edges so that the degree of $p_{[v]}$ in $T_{\Delta}$ is exactly $p \operatorname{deg}_{[v]}$. The degree of $p_{1}$ in $T_{\Delta}$ is $p \operatorname{deg}_{1}$.

There is one exceptional case when defining $T_{\Delta}$. If $n=5$ and $H$ has exactly three vertices, then the graph $H^{\prime}$ had exactly five vertices. Thus, there were exactly two children $\left[v_{0}^{\prime}\right]$ and $\left[\hat{v}_{0}^{\prime}\right]$ which had exactly half the number of descendants of $\left[v_{0}\right]$. The vertices of $H^{\prime}$ are then exactly $p_{1}, p_{\left[v_{0}\right]}, p_{\left[v_{0}^{\prime}\right]}, p_{\left[\hat{v}_{0}^{\prime}\right]}$, and some vertex $p_{[w]}$. Let $a, b$, and $c$ be positive integers greater than 2 such that

$$
Y_{2}(a)=\left|\left[v_{0}\right]\right|, \quad Y_{3}(b)-Y_{2}(b)=|[w]|, \quad Y_{2}(c)=\left|N_{w}\right|,
$$

if such integers exist. If any of $a, b$, or $c$ is undefined, then $T_{\Delta}$ is undefined. If $a$, $b$, and $c$ are all defined, then define $T_{\Delta}$ to be the linear tree having no vertices of degree 2 and exactly three essential vertices $A, B$, and $C$, where the essential vertices have degrees $a, b$, and $c$, respectively, and $A$ and $C$ are extremal.

We note that, according to the definition presented here, the tree $T_{\Delta}$ may depend on the choice of $\leq_{N}$-maximal vertex $v_{0}$. As a consequence of Theorem 6.9, we will have that, when $\Delta$ is the unique simplicial complex associated to $H^{*}\left(B_{n} T\right)$ for $T$ a tree and $n=4$ or 5 , the tree $T_{\Delta}$ is independent of $v_{0}$. 
Example 6.1. Consider the simplicial complex $\Delta$ shown in Figure 5, and reproduced in Figure 8. We have labelled the vertices of $\Delta$ with nontrivial neighborhoods. With the vertices of $\Delta$ as labelled in Figure 8, the vertex $v_{0}$ is maximal under $\leq_{N}\left(N_{v_{0}}=\right.$ $\left.\left\{v_{1}, v_{4}, v_{5}\right\}\right)$. Since $N_{v_{1}}=\left\{v_{4}\right\}$ and $N_{v_{2}}=\left\{v_{5}\right\},\left[v_{1}\right]$ and $\left[v_{2}\right]$ are descendants of $\left[v_{0}\right]$. The neighborhood hierarchy of $\left[v_{0}\right]$ is shown on the right of Figure 8 with solid edges. Fixing $n$ to be 4 , the tree $H$ constructed above is the neighborhood hierarchy. To grow $T_{\Delta}$ from $H$, we compute the desired degrees in $T_{\Delta}$ of the vertices of $H$. The desired degree of $p_{1}$ is 3 , since $Y_{2}(3)=1=\left|\left[v_{0}\right]\right|$. The desired degree of $p_{\left[v_{0}\right]}$ is $p \operatorname{deg}_{\left[v_{0}\right]}=3$, since $p_{\left[v_{0}\right]}$ is not a leaf of $H$ and $Y_{2}(3)=1=\left|\left[v_{1}\right]\right|=\left|\left[v_{2}\right]\right|$. The desired degree of $p_{\left[v_{1}\right]}$ is $p \operatorname{deg}_{\left[v_{1}\right]}=3$, since $p_{\left[v_{1}\right]}$ is a leaf of $H$ and $Y_{(4-2)}(3)=$ $1=\left|N_{v_{1}}\right|$. Finally, the desired degree of $p_{\left[v_{2}\right]}$ is $p \operatorname{deg}_{\left[v_{2}\right]}=3$, since $p_{\left[v_{2}\right]}$ is a leaf of $H$ and $Y_{(4-2)}(3)=1=\left|N_{v_{1}}\right|$. Thus, the tree $T_{\Delta}$ is as depicted on the right of Figure 8.
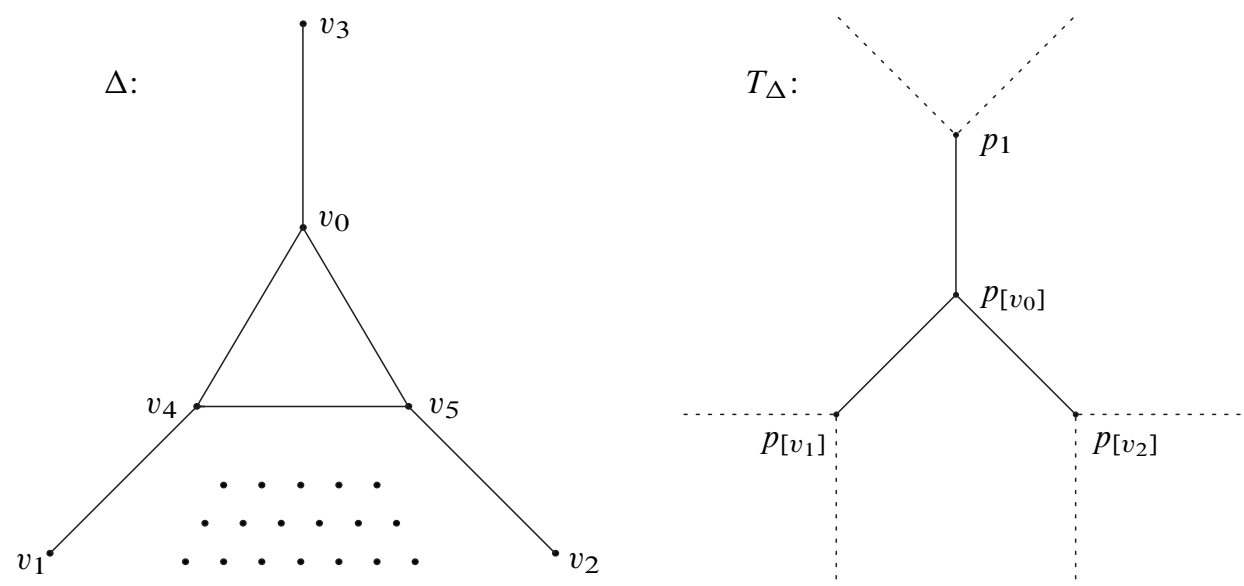

Figure 8. An example calculation of the tree $T_{\Delta}$.

6.2. The four and five strand case. We now wish to prove the rigidity for tree braid groups in the restricted setting of $n=4$ or 5 . As usual, we need a series of definitions and lemmas to complete the proof. These lemmas will completely describe, for a given critical cell $c$, which critical cells $c^{\prime}$ are such that $M c^{*} \cup M\left(c^{\prime}\right)^{*} \neq[0]$. It is this information that we use to prove Theorem 6.9. All lemmas in this subsection will assume $n \in\{4,5\}$.

For any critical 1-cell $c$, define the $M$-cup neighborhood $N_{c}$ of $c$ to be the set

$$
N_{c}:=\left\{c_{1} \mid M c^{*} \cup M c_{1}^{*} \neq[0]\right\} .
$$

When $\Delta$ is as in Theorem 5.15 and $v=M c^{*}$ is a vertex of $\Delta$, the vertex neighborhood $N_{v}$ of $v$ in $\Delta$ is by definition in bijective correspondence with the $M$-cup neighborhood of $c$. 
Lemma 6.2 (The direction $\left.d_{c}\right)$. Let $c=(a, e, \vec{x})$ be a critical 1-cell. Either the $M$-cup neighborhood $N_{c}$ of $c$ is empty or there exists a unique direction $d$ from a such that, for every $c_{1} \in N_{c}, c_{1}$ lies in direction $d_{c}$ from $a$.

The direction $d_{c}$ is called the CUB (Critical Upper Bound) direction for $c$.

Proof. This lemma follows from repeated application of the Upper Bound Lemma, Lemma 3.9. Throughout this proof, we use the Upper Bound Lemma, usually without reference.

Assume the converse: that $N_{c}$ is nonempty and there are critical 1-cells $c_{1}, c_{2} \in$ $N_{c}$ such that the directions $d_{1}$ and $d_{2}$ from $a$ toward $c_{1}$ and $c_{2}$, respectively, are distinct. By definition, we know $x_{d} \geq 1$. We have that $x_{d_{1}}, x_{d_{2}} \geq 2$. Also, if $d_{1}=d$ then $x_{d_{1}} \geq 3$, while if $d_{2}=d, x_{d_{2}} \geq 3$. If $x_{d_{1}} \geq 3$ and $d_{2} \neq d$, then $x_{d_{2}} \leq 1$, a contradiction. If $x_{d_{2}} \geq 3$ while instead $d_{2}=d$, then $x_{d_{1}} \leq 2$, again a contradiction. Thus, $x_{d_{1}}<3$. Since $2 \leq x_{d_{1}}<3, x_{d_{1}}=2$, and so $d_{1} \neq d$. Similarly, $x_{d_{2}}=2$ and $d_{2} \neq d$.

Consider if $d_{1}=0$. Then $c$ is not exceptional of Type I. Let $\left[s_{2}\right]$ be the least upper bound of $[c]$ and $\left[c_{2}\right]$, and let $s_{2}$ be the reduced representative of $\left[s_{2}\right]$. By Corollary 5.16, $s_{2}$ exists and is critical. But by the Upper Bound Lemma, $e$ is respectful in $s_{2}$, a contradiction. Thus, $d_{1} \neq 0$. Similarly, $d_{2} \neq 0$.

If $d_{1}>d$, note $c$ is not exceptional of Type I. Let $\left[s_{2}\right]$ be the least upper bound of $[c]$ and $\left[c_{2}\right]$, and let $s_{2}$ be the reduced representative of $\left[s_{2}\right]$. By Corollary 5.16, $s_{2}$ exists and is critical. but by the Upper Bound Lemma, $e$ is respectful in $s_{2}$, a contradiction. Thus, $d_{1}<d$. Similarly, $d_{2}<d$.

The only possibility left is that $c$ is exceptional of Type II. The existence of both $c_{1}$ and $c_{2}$ contradicts Lemma 5.13. This finishes the proof.

Consider a critical 1-cell $c=(a, d, \vec{x})$ with nonempty $M$-cup neighborhood $N_{c}$, and $d_{c}$ as in Lemma 6.2. Just as the CUB direction $d_{c}$ helps determine $N_{c}$, there is a number, $\operatorname{CUB}(c)$, that we may associate to $c$ to help determine $N_{c}$. Before defining $\operatorname{CUB}(c)$, we need the following definition. Let $\epsilon_{c}\left(d^{\prime}\right)$ be the cup constant of $c$ in direction $d^{\prime}$, associated to $c$ as follows. If $d^{\prime}=0$ or $d^{\prime}>d$, define $\epsilon_{c}\left(d^{\prime}\right):=0$. If $0<d^{\prime}<d$ and there is some index $i$ with $0<i<d$ and $i \neq d^{\prime}$ with $x_{i}>0$, define $\epsilon_{c}\left(d^{\prime}\right):=0$. If $n=5$ and $c$ is exceptional of Type I, define $\epsilon_{c}\left(d^{\prime}\right):=0$. Otherwise, define $\epsilon_{c}\left(d^{\prime}\right):=1$. Note that, unless $n=5$ and $c$ is exceptional of Type $\mathrm{I}$, if the upper bound constant $\epsilon\left(c, d^{\prime}\right)$ for $c$ in direction $d^{\prime}$ is 1 , then the cup constant $\epsilon_{c}\left(d^{\prime}\right)$ for $c$ in direction $d^{\prime}$ is also 1 . For convenience, we let $\epsilon_{c}:=\epsilon_{c}\left(d_{c}\right)$. Then, the number $x_{d_{c}}-\epsilon_{c}$ is denoted CUB $(c)$, and is the CUB (Critical Upper Bound) number for $c$.

Lemma 6.3 (A bound on CUB $(c)$ ). For a critical 1-cell c with nonempty $M$-cup neighborhood, $2 \leq \mathrm{CUB}(c) \leq n-2$. 
Proof. If $c$ is exceptional of Type I, then $\operatorname{CUB}(c)=2$ and there is nothing to prove. So, assume $c$ is not exceptional of Type I. Let $c=(a, d, \vec{x})$. By the Upper Bound Lemma, $x_{d_{c}} \geq 2+\epsilon\left(c, d_{c}\right)$. Since $c$ has non-empty $M$-cup neighborhood, this inequality is strict if there exists no index $i \neq d_{c}$ such that $0<i<d$ and $x_{i}>0$, by Corollary 5.16. But by the definition of $\epsilon_{c}$, this means $x_{d_{c}} \geq 2+\epsilon_{c}$. Thus,

$$
\operatorname{CUB}(c)=x_{d_{c}}-\epsilon_{c} \geq 2 .
$$

Since $c$ is critical, there exist at least two nonzero coordinates of $\vec{x}$, so $x_{d_{c}} \leq n-1$. If $x_{d_{c}}<n-1$, there is nothing to prove, so consider if $x_{d_{c}}=n-1$. If $d_{c}=0$ or $d_{c}>d$, then $c$ is not critical, so it must be that $0<d_{c} \leq d$. If $d_{c}=d$, then $\epsilon_{c}=1$. If $0<d_{c}<d$, then there exists no index $j \neq d_{c}$ with $0<j<d$ and $x_{j}>0$, so again $\epsilon_{c}=1$. Either way,

$$
\operatorname{CUB}(c)=x_{d_{c}}-\epsilon_{c} \leq n-1-1=n-2 .
$$

The CUB direction and number determine $N_{c}$ in the following sense:

Lemma 6.4 (The structure of neighborhoods). Let $c=(a, e, \vec{x})$ and $c^{\prime}=\left(a^{\prime}, e^{\prime}, \vec{x}^{\prime}\right)$ be critical 1-cells. Then $c \in N_{c^{\prime}}$ and $c^{\prime} \in N_{c}$ if and only if:

(1) $a \neq a^{\prime}$,

(2) $a^{\prime}$ lies in direction $d_{c}$ from $a$,

(3) a lies in direction $d_{c^{\prime}}$ from $a^{\prime}$, and

(4) $\operatorname{CUB}(c)+\operatorname{CUB}\left(c^{\prime}\right) \geq n$.

Proof. Note $c \in N_{c^{\prime}}$ if and only if $c^{\prime} \in N_{c}$ by definition. If $c \in N_{c^{\prime}}$ and $c^{\prime} \in N_{c}$, then the first three conditions follow from Lemma 6.2 and the fourth condition from the Upper Bound Lemma, Lemma 3.9.

Now consider if the four conditions hold. That $[c]$ and $\left[c^{\prime}\right]$ have an upper bound $[s]$ follows from the Upper Bound Lemma. Let $s$ be the reduced representative of $[s]$. We claim that either $s$ is critical or the $\leq_{N}$-smaller of $c$ and $c^{\prime}$ is exceptional of Type I. If the claim holds, then by Corollary 5.16, $c \in N_{c^{\prime}}$ and $c^{\prime} \in N_{c}$. If $s$ is not critical, then one of $e$ or $e^{\prime}$ is respectful in $s$. Without loss of generality, assume $e$ is respectful in $s$. By the Upper Bound Lemma, $d_{c^{\prime}}=0$ and $d_{c}$ satisfies $0<d_{c}<$ dir, where dir is the direction from $a$ along $e$. Furthermore, $x_{i}=0$ for every $i \neq d_{c}$ satisfying $0<i<\mathrm{dir}$, and $x_{d_{c}}+x_{0}^{\prime}=n$. By definition, $\epsilon_{c^{\prime}}\left(d_{c^{\prime}}\right)=0$. Since

$$
n-\epsilon_{c}=\left(x_{d_{c}}-\epsilon_{c}\right)+\left(x_{0}^{\prime}-\epsilon_{c}^{\prime}\right)=\operatorname{CUB}(c)+\operatorname{CUB}\left(c^{\prime}\right) \geq n,
$$

it follows that $\epsilon_{c}=0$. By definition, it must be that $c$ is exceptional of Type I. This proves the claim.

Corollary 6.5 (Neighborhood containment). Let $c=(a, d, \vec{x})$ and $c^{\prime}=\left(a^{\prime}, d^{\prime}, \overrightarrow{x^{\prime}}\right)$ be critical 1-cells with nonempty $M$-cup neighborhoods. Then $N_{c^{\prime}} \subseteq N_{c}$ if and only if $\mathrm{CUB}\left(c^{\prime}\right) \leq \mathrm{CUB}(c)$ and either: 
(1) $a=a^{\prime}$ and $d_{c}=d_{c^{\prime}}$, or

(2) $a \neq a^{\prime}$ and

(a) $a^{\prime}$ is in direction $d_{c}$ from $a$, and

(b) $a$ is not in direction $d_{c^{\prime}}$ from $a^{\prime}$.

Proof. These conditions are equivalent to the statement "if $c_{0} \in N_{c^{\prime}}$ then $c_{0} \in N_{c}$ ", by Lemma 6.4 .

Corollary 6.6 (Neighborhood equality). Let $c=(a, d, \vec{x})$ and $c^{\prime}=\left(a^{\prime}, d^{\prime}, \vec{x}^{\prime}\right)$ be critical 1-cells with nonempty $M$-cup neighborhoods. Then $N_{c}=N_{c^{\prime}}$ if and only if

(1) $a=a^{\prime}$,

(2) $d_{c}=d_{c^{\prime}}$, and

(3) $\operatorname{CUB}(c)=\operatorname{CUB}\left(c^{\prime}\right)$.

Proof. That $N_{c}=N_{c^{\prime}}$ is equivalent to $N_{c} \subseteq N_{c^{\prime}}$ and $N_{c^{\prime}} \subseteq N_{c}$. The result then follows from applying Corollary 6.5 twice.

This lemma and its two corollaries almost completely describe the structure of the $M$-cup neighborhoods as well as their behavior under inclusion. The final two ingredients for the proof of Theorem 6.9 are to count exactly how many critical 1cells have a given neighborhood, and describe what kinds of critical 1-cells have neighborhoods which are maximal under inclusion. We accomplish these tasks in the following two results.

Lemma 6.7 (Counting). For any essential vertex a, there exist exactly $Y_{n}(\operatorname{deg}(a))$ critical 1-cells lying over $a$. Let $d$ be a direction from a such that there exists some essential vertex besides a itself in direction d from $a$. Let $k$ be an integer satisfying $2 \leq k \leq n-2$. Then there exists exactly $Y_{n-k}(\operatorname{deg}(a))$ critical 1 -cells lying over a with CUB direction $d$ and CUB number at least $k$.

Proof. By Definition 3.7 and the preceding discussion, a critical 1-cell $c$ is determined by: an essential vertex $a$, an edge $e$ with terminal endpoint $a$, an $a$-vector $\vec{x}$, and the condition that at least one vertex of $c$ be blocked by $e$ at $a$. All of these properties are local properties, and do not depend on $T$ or on the existence or nonexistence of any other essential vertices in $T$. Thus, there is a bijection between critical 1cells lying over $a$ and critical 1-cells in a radial tree whose essential vertex has degree $\operatorname{deg}(a)$. The first statement of the lemma then follows from the Radial Rank Theorem, Theorem 3.4.

We now prove the second statement of the lemma. If $c=(a, \operatorname{dir}, \vec{x})$ is a critical 1-cell lying over $a$ with $d_{c}=d$ and $\operatorname{CUB}(c) \geq k$, then $x_{d} \geq k+\epsilon_{c}$. Thus, $c$ has at least $k$ vertices (not including the edge $e$ ) in direction $d$ from $a$. Let $c^{\prime}$ be the reduced 1-cell corresponding to deleting $k$ vertices from $c$ in direction $d$ from $a$. That is, 
$c^{\prime}:=\left(a\right.$, dir, $\left.\vec{x}^{\prime}\right)$, where $\vec{x}^{\prime}$ equals $\vec{x}$ in all but the $d^{\text {th }}$ coordinate, and $x_{d}^{\prime}=x_{d}-k$. We make one exception: if $c$ is exceptional of Type I, define $c^{\prime}:=\left(a, \operatorname{dir}^{\prime}, \vec{x}^{\prime}\right)$, where $\operatorname{dir}^{\prime}>$ dir is the direction towards the edge of the exceptional 1-cell of Type II associated to $c$.

We claim $c^{\prime}$ is critical. For, if $c^{\prime}$ is not critical, then it must be that $0<d<\mathrm{dir}$, $x_{d}=k$, and $x_{i}=0$ for all $0<i<\operatorname{dir}, i \neq d$. Since $x_{d} \geq k+\epsilon_{c}$, it must be that $\epsilon_{c}=0$. By the definition of $\epsilon_{c}$, it follows that $c$ is exceptional of Type I. But when $c$ is exceptional of Type $\mathrm{I}$, we changed $c^{\prime}$ to be critical.

Now consider a critical 1-cell $c_{0}^{\prime}$ lying over $a$ on $n-k$ strands. Let $c_{0}$ be the reduced 1-cell on $n$ strands corresponding to adding $k$ vertices to $c_{0}^{\prime}$ in the direction $d$ from $a$. As $c_{0}^{\prime}$ is critical, so is $c_{0}$. It is straightforward to construct a critical 1-cell $c_{1}$ lying over a vertex in direction $d$ from $a$ such that $\left[c_{0}\right]$ and $\left[c_{1}\right]$ have a least upper bound $[s]$ whose reduced representative $s$ is critical. We leave this to the reader. By Lemma 6.2 and Corollary 5.16, unless $c_{0}$ is exceptional of Type II and $d$ is the smallest direction from $a$ in which $c_{0}$ has strands, $d_{c_{0}}=d$. It then follows that $\operatorname{CUB}\left(c_{0}\right) \geq k$. Furthermore, since $c_{0}^{\prime}$ is critical, $c_{0}$ cannot be of Type I. If $c_{0}$ is exceptional of Type II and $d$ is the smallest direction from $a$ in which $c_{0}$ has strands, we make an exception and replace $c_{0}$ with the corresponding exceptional cell of Type I.

These two constructions (constructing $c^{\prime}$ from $c$ and $c_{0}$ from $c_{0}^{\prime}$ ) are inverses of each other (we leave it to the reader to check this when $c$ is of Type I or $c_{0}^{\prime}$ is of Type II). Thus, they give a set bijection between 'critical 1-cells $c$ with $d_{c}=d$ and $\operatorname{CUB}(c) \geq k$ ' and 'critical 1-cells lying over $a$ with $n-k$ strands'. In particular, these two sets have the same number of elements. The latter set has $Y_{n-k}(\operatorname{deg}(a))$ elements, by Theorem 3.4. This proves the lemma.

When $\Delta$ is as in Theorem 5.15 and $v=M c^{*}$ is a vertex of $\Delta$ where $[v]$ is maximal under $\leq_{N}$, then both the vertex neighborhood $N_{v}$ and the $M$-cup neighborhood $N_{c}$ will be maximal under inclusion. Furthermore:

Corollary 6.8 (Maximal neighborhoods). Let c be a critical 1-cell. If the $M$-cup neighborhood $N_{c}$ of $c$ is maximal under inclusion among all $M$-cup neighborhoods, then $c$ lies over an extremal vertex a of $T$, and $\mathrm{CUB}(c)=n-2$.

Proof. Let $a$ be the essential vertex over which $c$ lies. If $T$ is radial, then the only essential vertex is $a$, so $a$ is extremal. If $T$ is not radial, then there exist non-empty $M$-cup neighborhoods, so the empty set is not maximal under inclusion and therefore $N_{c}$ is nonempty. Assume that $a$ is not extremal, and let $d_{c}$ be the direction prescribed by Lemma 6.2. Since $a$ is not extremal, there exist extremal vertices in at least 2 directions from $a$. Thus there exists an extremal vertex $a^{\prime}$ in a direction which is not $d_{c}$ from $a$. Let $d^{\prime}$ be the direction from $a^{\prime}$ to $a$. By Lemma 6.7, there exists a critical 1-cell $c^{\prime}$ lying over $a^{\prime}$ with $d_{c^{\prime}}=d^{\prime}$ and $\operatorname{CUB}\left(c^{\prime}\right)=\operatorname{CUB}(c)$. By Corollaries 6.5 and 6.6, $N_{c} \subsetneq N_{c^{\prime}}$, contradicting the maximality of $N_{c}$. Thus $a$ is extremal. 
That $\operatorname{CUB}(c)=n-2$ is similar: assume $\operatorname{CUB}(c)<n-2$. By Lemma 6.7, there exists a critical 1-cell $c^{\prime}$ lying over $a$ with $d_{c^{\prime}}=d_{c}$ and $\operatorname{CUB}\left(c^{\prime}\right)=n-2$. By Corollaries 6.5 and 6.6, $N_{c} \subsetneq N_{c^{\prime}}$, contradicting the maximality of $N_{c}$. Thus $\operatorname{CUB}(c)=n-2$.

We are now ready to prove the following rigidity result for $n=4$ and 5 strand tree braid groups:

Theorem 6.9 (Rigidity). Let $T$ and $T^{\prime}$ be two finite trees, and let $n=4$ or 5 . The tree braid groups $B_{n} T$ and $B_{n} T^{\prime}$ are isomorphic as groups if and only if the trees $T$ and $T^{\prime}$ are homeomorphic as trees.

There are many equivalent formulations of Theorem 6.9. Some of these restatements include:

- there is a bijection between finite trees up to homeomorphism and $n$-strand tree braid groups up to isomorphism, $n=4$ or 5;

- there is a bijection between $n$-strand tree braid groups up to isomorphism, $n=4$ or 5 , and the set of cohomology rings of $n$ strand tree braid groups (that this is a reformulation follows from Theorem 5.2);

- Given an $n$-strand tree braid group $G$ with $n=4$ or 5 , there exists a unique tree $T$ (up to homeomorphism) such that $G=B_{n} T$.

To prove Theorem 6.9, we actually show the last equivalent statement.

Note that, in each homeomorphism class of trees, there exists exactly one tree that has no vertices of degree two. For this tree, the vertices are exactly all of the nonmanifold points. The following proof involves construction of a tree with no vertices of degree two, and graph braid groups on this tree. As the fundamental group of the associated configuration spaces is invariant under homeomorphism, this does not affect the associated tree braid group. The reader is cautioned, however, that in order to utilize critical cells via discretized configuration spaces and Theorem 2.2, further subdivision of this tree is required.

For applications of the proof of Theorem 6.9, we refer the reader to Examples 6.10 and 6.11 , immediately following the proof.

Proof. Let Trees denote the set of all trees up to homeomorphism. Let $B_{n}$ be the map taking a tree $T$ to its $n$ strand tree braid group $B_{n} T$. By definition, $B_{n}$ is well defined. We prove $B_{n}$ : Trees $\rightarrow B_{n}$ (Trees) is bijective for $n=4,5$ by constructing an inverse map. Let $\Psi_{n}: B_{n}$ (Trees) $\rightarrow$ Trees be given by $\Psi_{n}\left(B_{n} T\right)=T_{\Delta}$, where $\Delta$ is the simplicial complex uniquely determined by $H^{*}\left(B_{n} T\right)$, as in Theorem 5.2, and $T_{\Delta}$ is as in Section 6.1. We claim $\Psi_{n}$ is well defined and that $\Psi_{n} \circ B_{n}$ is the identity map for $n=4,5$. Our goal is to analyze $\Psi_{n}$ so that both properties will be proven simultaneously. This would prove the desired result, as $B_{4}$ and $B_{5}$ would thus be injective. See Figure 9. 


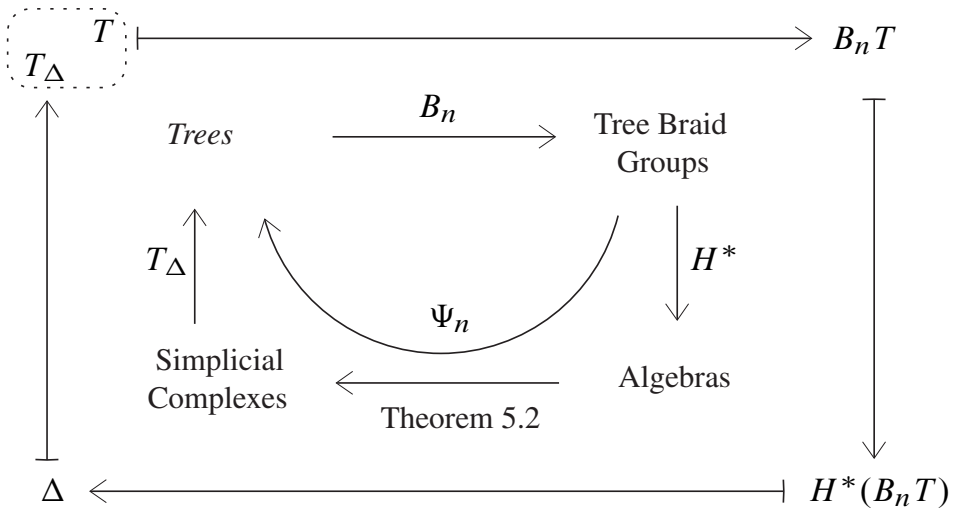

Figure 9. A diagram showing the maps used in the proof of Theorem 6.9. The proof of Theorem 6.9 shows that, when $n=4$ or $5, T_{\Delta}$ is homeomorphic to $T$.

Let $T$ be a tree. If $T$ is radial with central vertex $a$, then by [16] we know that $H^{*}\left(B_{n} T\right)$ is free of rank $Y_{n}(\operatorname{deg}(a))$ (see Theorem 3.4). Then $\Delta$ is a collection of $Y_{n}(\operatorname{deg}(a))$ vertices. By definition, $T_{\Delta}$ is a tree with one essential vertex of degree $\operatorname{deg}(a)$. Thus, $T$ is homeomorphic to $T_{\Delta}$, as desired.

Assume that $T$ is not radial (and is sufficiently subdivided for $n$ ). Let $\Delta$ be the unique 1-dimensional simplicial complex giving the exterior face algebra structure on $H^{*}\left(B_{n} T\right)$ given by Theorem 5.2. Fix a Morse $T$-embedding, so that the vertices of $\Delta$ may be labelled $M c^{*}$, where each $c$ is a critical 1-cell, as in Definition 5.14.

Since $T$ is not radial and $n \geq 4$, there exist critical 2-cells (this is a consequence of Lemmas 6.4 and 6.7, and is an exercise for the reader). By Theorem 3.6, $H^{2}\left(B_{n} T\right)$ is nontrivial, so $\Delta$ contains some vertex with nonempty vertex neighborhood. Let $v_{0}=M c_{0}^{*}$ be a vertex of $\Delta$ which is maximal under $\leq_{N}$. We will prove that the tree $T_{\Delta}$ defined by this choice of $\leq_{N}$-maximal vertex is homeomorphic to $T$.

By the definition of $\leq_{N}$, both the vertex neighborhood $N_{v_{0}}$ of $v_{0}$ and the $M$-cup neighborhood $N_{c_{0}}$ of $c_{0}$ are nonempty and maximal under inclusion. Let $q_{1}$ denote the essential vertex in $T$ over which $c_{0}$ lies. By Corollary 6.8, $q_{1}$ is extremal and $\operatorname{CUB}(c)=n-2$.

Consider a descendant $[v]$ of $\left[v_{0}\right]$. Pick $v \in[v]$, and let $c$ be the critical 1-cell such that $v=M c^{*}$. Let $q$ be the vertex over which $c$ lies. By Lemma 6.3, we have that $2 \leq \mathrm{CUB}(c) \leq n-2$. By Corollary 6.5, either

(1) $n=5, q=q_{1}, d_{c}=d_{c_{0}}$, and $\operatorname{CUB}(c)=\operatorname{CUB}\left(c_{0}\right)-1=2$, or

(2) $q \neq q_{1}, q$ is in direction $d_{c_{0}}$ from $q_{1}$, and $q_{1}$ is not in direction $d_{c}$ from $q$.

Consider if $\operatorname{CUB}(c)=\operatorname{CUB}\left(c_{0}\right)=n-2$. Then we are in the second case above. Label the unique essential vertex of $T$ which is adjacent to $q$ and is in direction $d_{c}$ from $q$ by $q_{[v]}$. By Corollary 6.6, the vertex $q$, the CUB direction $d_{c}$, and the CUB 
number $\operatorname{CUB}(c)$ are independent of the choice of $v \in[v]$, so $q_{[v]}$ is well defined. By Lemma 6.7, every essential vertex of $T$ except $q_{1}$ will have a label of the form $q_{[v]}$. As $T$ is a tree, the labels are unique.

We claim that the map $q_{\left[M c^{*}\right]} \mapsto p_{\left[M c^{*}\right]}$ from essential vertices of $T$ to vertices of $T_{\Delta}$ shows that $T_{\Delta}$ is defined and that this map induces the desired homeomorphism $\Psi_{n}$ between the two trees. To prove our claim, we need to show:

(i) There exists a vertex labelled $q_{\left[M c^{*}\right]}$ in $T$ if and only if there exists a vertex labelled $p_{\left[M c^{*}\right]}$ in $T_{\Delta}$,

(ii) $q_{\left[M\left(c^{\prime}\right)^{*}\right]}$ is adjacent to $q_{\left[M c^{*}\right]}$ if and only if $p_{\left[M\left(c^{\prime}\right)^{*}\right]}$ is adjacent to $p_{\left[M c^{*}\right]}$, and (iii) $\operatorname{deg}\left(q_{\left[M c^{*}\right]}\right)=\operatorname{deg}\left(p_{\left[M c^{*}\right]}\right)$.

(i) If $n=4$, this is clear. If $n=5$, let $\left[v_{0}^{\prime}\right]$ be a child of $\left[v_{0}\right]$, and let $c_{0}^{\prime}$ be a critical 1-cell such that $v_{0}^{\prime}=M^{*} c_{0}^{\prime}$. Consider if $\operatorname{CUB}\left(c_{0}^{\prime}\right)=\operatorname{CUB}\left(c_{0}\right)-1=2$. For $\left[v_{0}^{\prime}\right]$ to be a child of $\left[v_{0}\right]$, it follows from Corollary 6.5 that $c_{0}^{\prime}$ lies over $q_{1}$ and $d_{c_{0}^{\prime}}=d_{c_{0}}$. By Corollary 6.6, $\left[v_{0}^{\prime}\right]$ is thus uniquely determined by the choice of $\operatorname{CUB}\left(c_{0}^{\prime}\right)=2$. Either a descendant $\left[M c^{*}\right]$ of $\left[v_{0}\right]$ is also a descendant of $\left[v_{0}^{\prime}\right]$ or not. By Corollary 6.5 , $\left[M c^{*}\right]$ is a descendant of $\left[v_{0}^{\prime}\right]$ if and only if $\operatorname{CUB}(c)=2$, and is not a descendant of $\left[v_{0}^{\prime}\right]$ if and only if $\operatorname{CUB}(c)=3$. Thus, by Lemma $6.7,\left[v_{0}^{\prime}\right]$ has exactly half as many descendants as $\left[v_{0}\right]$. If $\left[M c^{*}\right]$ and $\left[M\left(c^{\prime}\right)^{*}\right]$ are distinct children of $\left[v_{0}\right]$ such that neither $\left[M c^{*}\right]$ nor $\left[M\left(c^{\prime}\right)^{*}\right]$ is $\left[v_{0}^{\prime}\right]$, then $\operatorname{CUB}(c)=\operatorname{CUB}\left(c^{\prime}\right)=\operatorname{CUB}\left(c_{0}\right)=3$, as $\left[v_{0}^{\prime}\right]$ is uniquely determined by the choice $\operatorname{CUB}\left(c_{0}^{\prime}\right)=2$. By Corollary 6.5 , it follows that $c$ and $c^{\prime}$ both lie over $q_{\left[v_{0}\right]}$. By Corollary 6.6 , since $\left[M c^{*}\right] \neq\left[M\left(c^{\prime}\right)^{*}\right]$, $d_{c} \neq d_{c}^{\prime}$. It follows from Corollary 6.5 that $\left[M c^{*}\right]$ and $\left[M\left(c^{\prime}\right)^{*}\right]$ have no common descendants, let alone children.

Thus, $\left[v_{0}^{\prime}\right]$ is as in the definition of $T_{\Delta}$. Note this child $\left[v_{0}^{\prime}\right]$ always exists, by Lemma 6.7. There exists a vertex labelled $q_{\left[M c^{*}\right]}$ in $T$ if and only if $\left[M c^{*}\right]$ is a descendant of $\left[v_{0}\right]$ and $\operatorname{CUB}(c)=3$, if and only if $\left[M c^{*}\right]$ is a descendant of $\left[v_{0}\right]$ but not $\left[v_{0}^{\prime}\right]$, if and only if there exists a vertex labelled $p_{\left[M c^{*}\right]}$ in $T_{\Delta}$. This proves (i) in the case that $\operatorname{CUB}\left(c_{0}^{\prime}\right)=2$.

If $\operatorname{CUB}\left(c_{0}^{\prime}\right)=3$, let $\left[M\left(c^{\prime}\right)^{*}\right]$ denote the child of $\left[v_{0}\right]$ such that $\operatorname{CUB}\left(c^{\prime}\right)=2$. If $\left[v_{0}\right]$ has any other child $\left[M c^{*}\right]$ distinct from $\left[v_{0}^{\prime}\right]$ and $\left[M\left(c^{\prime}\right)^{*}\right]$, then $\left[M\left(c^{\prime}\right)^{*}\right]$ and $\left[M c^{*}\right]$ have a common descendant (namely, $\left[M\left(c^{\prime \prime}\right)^{*}\right]$ where $\operatorname{CUB}\left(c^{\prime \prime}\right)=2$, $d_{c^{\prime \prime}}=d_{c}$, and $c^{\prime \prime}$ and $c$ lie over the same vertex in $T$ ). Thus, unless $\left[v_{0}\right]$ has no other children, $\left[v_{0}^{\prime}\right]$ is not as in the definition of $T_{\Delta}$. If $\left[v_{0}\right]$ has no other children, then by Corollary 6.5, every descendant of $\left[v_{0}\right]$ except $\left[v_{0}\right]$ and $\left[M\left(c^{\prime}\right)^{*}\right]$ are also descendants of $\left[v_{0}^{\prime}\right]$. Thus, unless $\left[v_{0}\right]$ has exactly 4 descendants, $\left[v_{0}^{\prime}\right]$ is again not as in the definition of $T_{\Delta}$.

If $\operatorname{CUB}\left(c_{0}^{\prime}\right)=3,\left[v_{0}\right]$ has no other children, and exactly 4 descendants, we are in the exceptional case in the definition of $T_{\Delta}$. We repeatedly apply Corollary 6.5 to analyze this case. As $\left[v_{0}\right]$ has only one child $\left[v_{0}^{\prime}\right]=\left[M\left(c_{0}^{\prime}\right)^{*}\right]$ with $\operatorname{CUB}\left(c_{0}^{\prime}\right)=3$, the vertex $q_{\left[v_{0}\right]}$ is adjacent to exactly two essential vertices in $T: q_{1}$ and $q_{\left[v_{0}^{\prime}\right]}$. As $\left[v_{0}^{\prime}\right]$ has only one descendant $\left[M c^{*}\right], q_{\left[v_{0}^{\prime}\right]}$ must be adjacent to only $q_{\left[v_{0}\right]}$. Let $x, y$, and $z$ 
denote the degrees of vertices $q_{1}, q_{\left[v_{0}\right]}$, and $q_{\left[v_{0}^{\prime}\right]}$, respectively. Then by Lemma 6.7, it follows that:

$$
Y_{2}(x)=\left|\left[v_{0}\right]\right|, \quad Y_{3}(y)-Y_{2}(y)=\left|\left[M c^{*}\right]\right|, \quad Y_{2}(z)=\left|N_{c}\right| .
$$

Thus, in this exceptional case, we already have that $T$ is homeomorphic to $T_{\Delta}$. This finishes the proof of (i), as either we must be in this exceptional case or $\operatorname{CUB}\left(c_{0}^{\prime}\right)=2$.

(ii) Let $v:=M c^{*}$ and $v^{\prime}:=M\left(c^{\prime}\right)^{*}$ be such that $q_{[v]}$ and $q_{\left[v^{\prime}\right]}$ are essential vertices in $T$. Without loss of generality, assume $q_{[v]}$ is closer to $q_{1}$ than $q_{\left[v^{\prime}\right]}$. Since $\operatorname{CUB}(c)=\operatorname{CUB}\left(c^{\prime}\right)=n-2$, by Corollary $6.5, q_{[v]}$ and $q_{\left[v^{\prime}\right]}$ are adjacent if and only if there are no critical 1-cells $c^{\prime \prime}$ such that $N_{c^{\prime}} \subsetneq N_{c^{\prime \prime}} \subsetneq N_{c}$. By definition, this holds if and only if $\left[v^{\prime}\right]$ is a child of $[v]$, if and only if $p_{[v]}$ is adjacent to $p_{\left[v^{\prime}\right]}$.

(iii) Let $c$ be a critical 1-cell such that $q_{\left[M c^{*}\right]}$ labels a vertex of $T$. Let $a^{\prime}:=q_{\left[M c^{*}\right]}$, and let $a$ denote the vertex over which $c$ lies. Then $a^{\prime}$ is adjacent to $a$ and in direction $d_{c}$ from $a$. If $a^{\prime}$ is extremal, then by Lemma 6.4, $c^{\prime} \in N_{c}$ if and only if $c^{\prime}$ lies over $a^{\prime}$ and $d_{c^{\prime}}$ is the direction from $a^{\prime}$ to $a$. By Lemma 6.7, we have

$$
Y_{(n-2)}\left(\operatorname{deg}\left(a^{\prime}\right)\right)=\left|N_{c}\right| .
$$

If $a^{\prime}$ is not extremal, then if follows from Corollary 6.5 that any child $\left[M\left(c^{\prime}\right)^{*}\right]$ of $\left[M c^{*}\right]$ will satisfy either:

(1) $n=5, c^{\prime}$ lies over $a, d_{c^{\prime}}=d_{c}$, and $\operatorname{CUB}\left(c^{\prime}\right)=\operatorname{CUB}(c)-1=2$, or

(2) $c^{\prime}$ lies over $a^{\prime}, \operatorname{CUB}\left(c^{\prime}\right)=\operatorname{CUB}(c)$, and $a$ is not in direction $d_{c^{\prime}}$ from $a^{\prime}$.

In the former case, there is no vertex labelled $q_{\left[M\left(c^{\prime}\right)^{*}\right]}$ in $T$. In the latter case, by Lemma 6.7, we have

$$
Y_{2}\left(\operatorname{deg}\left(a^{\prime}\right)\right)=\left|N_{c^{\prime}}\right| .
$$

Thus, regardless of whether $a^{\prime}$ is extremal or not,

$$
\operatorname{deg}\left(q_{\left[M c^{*}\right]}\right)=\operatorname{deg}\left(p_{\left[M c^{*}\right]}\right) .
$$

This finishes the proof of (iii), and of the lemma.

Example 6.10. Consider the tree $T_{\min }$, shown on the right of Figure 10. On the left of Figure 10 is the tree $T_{\Delta}$ generated in Example 6.1. The complex $\Delta$ used is in fact the simplicial complex underlying the exterior face algebra structure on $H^{*}\left(B_{4} T_{\min }\right)$. The proof of Theorem 6.9 gives the isomorphism between $T_{\Delta}$ and $T$, induced by the map $q_{x} \mapsto p_{x}$. Here, $c_{0}=\left(q_{1}, 2,\left[\begin{array}{l}2 \\ 1 \\ 1\end{array}\right]\right), c_{1}=\left(q_{\left[v_{0}\right]}, 2,\left[\begin{array}{l}0 \\ 3 \\ 1\end{array}\right]\right)$, and $c_{1}=\left(q_{\left[v_{0}\right]}, 2,\left[\begin{array}{l}2 \\ 1 \\ 1\end{array}\right]\right)$.

Example 6.11. We wish to show an example of a partial computation for an $n=5$ strand tree braid group. Consider the tree $T$ shown on the left of Figure 11, depicted 

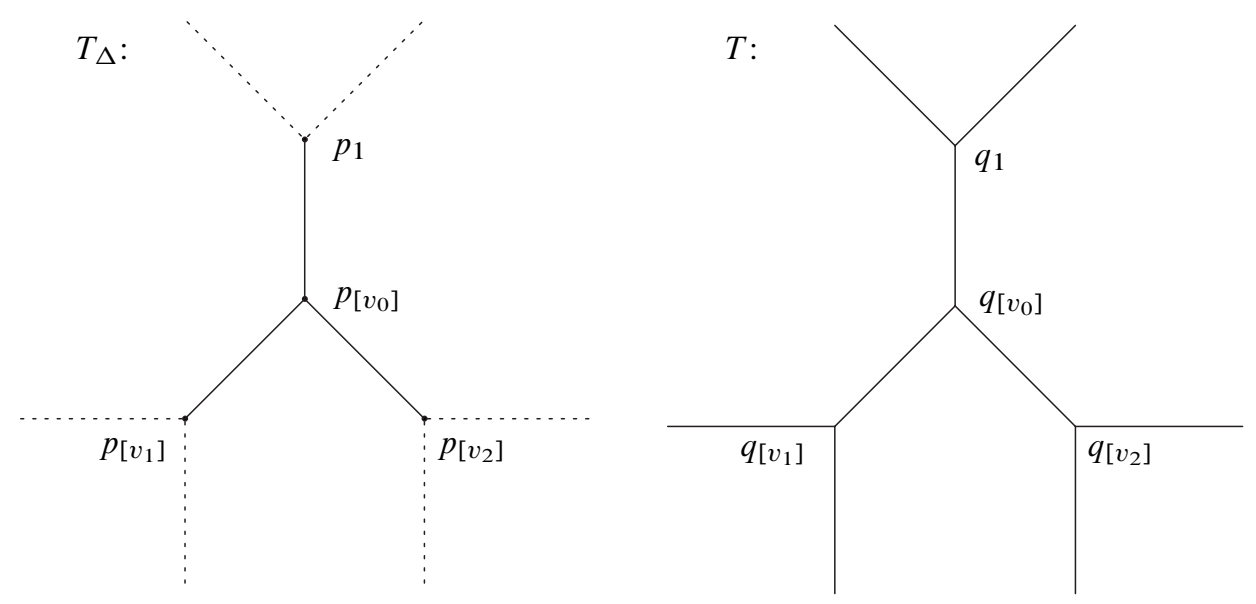

Figure 10. The tree $T_{\Delta}$ and the isomorphism of Theorem 6.9 for the tree $T_{\min }$.
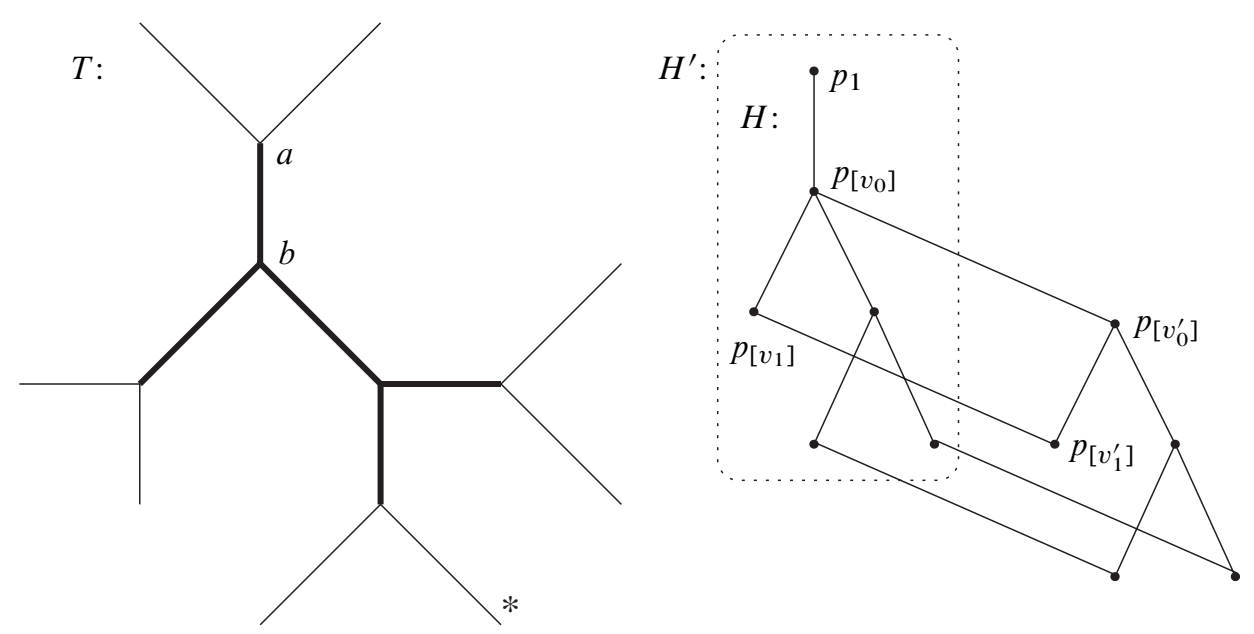

Figure 11. An example with $n=5$.

with a Morse $T$-embedding. Let $\Delta$ be the simplicial complex giving the exterior face algebra structure on $H^{*}\left(B_{5} T\right)$. On the right of Figure 11 is the graph $H^{\prime}$ is the neighborhood hierarchy $H^{\prime}$ for a vertex $v_{0}$ in the simplicial complex $\Delta$. Since we have a Morse $T$-embedding, we can give $v_{0}$ a label: it is $M c_{0}^{*}$, where $c_{0}$ is the critical 1-cell $\left(a, 2,\left[\begin{array}{l}3 \\ 1 \\ 1\end{array}\right]\right)$. Similarly, $v_{0}^{\prime}=M\left(c_{0}^{\prime}\right)^{*}, v_{1}=M c_{1}^{*}$, and $v_{1}^{\prime}=M\left(c_{1}^{\prime}\right)^{*}$, where $c_{0}^{\prime}:=\left(a, 2,\left[\begin{array}{l}2 \\ 1 \\ 2\end{array}\right]\right), c_{1}:=\left(b, 2,\left[\begin{array}{l}0 \\ 4 \\ 1\end{array}\right]\right)$, and $c_{1}^{\prime}:=\left(b, 2,\left[\begin{array}{l}0 \\ 3 \\ 2\end{array}\right]\right)$. The subtree $H$ of 
$H^{\prime}$, circled in Figure 11, is the subtree from which $T_{\Delta}$ is grown. The subtree $H$ is isomorphic to a subtree of $T$, shown in bold.

We may expand the proof of Theorem 6.9 to give us the following useful corollary:

Corollary 6.12 (Determining $n$ ). If $G=B_{n} T$ is a tree braid group on either $n=4$ or 5 strands for which $T$ has at least 3 essential vertices, then $n$ may be determined from $G$.

Proof. Let $\Delta$ be the unique simplicial complex associated to $H^{*}(G)$, by Theorem 5.2. If $T$ has at least 3 essential vertices, $T$ is not radial, so $G$ is not free. If $G$ is not free, there exists a vertex $v_{0}$ in the associated complex $\Delta$ with nonempty neighborhood and which is maximal under $\leq_{N}$. If $n=5$, then $\left[v_{0}\right]$ has a child $\left[v_{0}^{\prime}\right]$ as in the definition of $\Delta$. As $v_{0}$ is maximal under $\leq_{N}, v_{0}$ lies over an extremal vertex $q_{1}$ of $T$. Let $q_{\left[v_{0}\right]}$ denote the unique essential vertex of $T$ adjacent to $q_{1}$. As $T$ has at least 3 essential vertices, there exists a third essential vertex $q \neq q_{1}$ adjacent to $q_{\left[v_{0}\right]}$. Pick a Morse $T$-embedding, and let $c$ and $c^{\prime}$ be critical 1-cells that lie over $q_{\left[v_{0}\right]}$ and such that $d_{c^{\prime}}=d_{c}$ is the direction from $q_{\left[v_{0}\right]}$ to $q, \operatorname{CUB}(c)=3$, and $\operatorname{CUB}\left(c^{\prime}\right)=2$ (by Lemma 6.7, such $c$ and $c^{\prime}$ exist). By Corollary 6.5, it follows that $\left[M c^{*}\right]$ is a child of $\left[v_{0}\right]$ but not of $\left[v_{0}^{\prime}\right]$, and $\left[M\left(c^{\prime}\right)^{*}\right]$ is a child of both $\left[M c^{*}\right]$ and $\left[v_{0}^{\prime}\right]$. Thus, if $n=5$, there exist two children of [ $\left.v_{0}\right]$ with a common child. If $n=4$, then the neighborhood hierarchy of $\left[v_{0}\right]$ must be a tree, by Theorem 6.9. Thus, $n=5$ if and only if there exist two children of $\left[v_{0}\right]$ with a common child. Note this characterization does not actually depend on fixing a Morse $T$-embedding.

It seems reasonable that some combinatorial argument can address the case when $T$ has exactly two essential vertices. If this is done, the only exception to Corollary 6.12 would be when $T$ has exactly one essential vertex. But if $T$ has only one essential vertex, $T$ is radial, so $G$ is free. Thus, modulo a combinatorial argument about when $T$ has exactly two essential vertices, Corollary 6.12 would read: If $G$ is a tree braid group on either $n=4$ or 5 strands which is not free, then $n$ may be determined by $G$.

Theorem 6.9 also proves:

Corollary 6.13. The tree $T_{\Delta}$ constructed in Section 6.1 is defined if and only if $\Delta$ is a simplicial complex giving the exterior face algebra structure of $H^{*}\left(B_{n} T\right)$ for some tree $T$ and some $n=4$ or 5 .

The fact that one may reconstruct any tree $T$ up to homeomorphism given a (4 or 5 strand) tree braid group $B_{n} T$ on $T$ is an artifact of the 1-dimensionality of trees. For instance, let $k>1$ and consider the braid groups on a $k$-dimensional ball, and on a $k$-dimensional ball joined at a single point with a line segment. On any number of strands, the two corresponding braid groups are isomorphic. 
6.3. More strands. It would be nice to generalize Theorem 6.9 to say that, given a tree braid group on $n$ strands for any $n \geq 4$, one may reconstruct the underlying tree. As noted in Conjecture 5.17, we believe that tree braid groups on more than 5 strands do not have an exterior face algebra structure on cohomology in general. Thus the techniques used for $n=4$ and 5 probably do not apply for $n \geq 6$. However, we still believe a generalization is possible:

Conjecture 6.14 (Rigidity for an arbitrary number of strands). Let $G$ be an $n$ strand tree braid group where $n \geq 4$. Then there exists a unique tree $T$ (up to homeomorphism) such that $G=B_{n} T$.

Our intuition for conjecturing this generalization comes from the following situation. Let $T$ be a finite tree and let $n \geq 4$. Fix a Morse $T$-embedding. As we have a Morse $T$-embedding, we may talk about critical cells. We may still apply the results of Sections 3 and 4 to $T$ and its critical cells. Consider a critical 1-cell $c_{0}=\left(a_{0}, d_{0}, \vec{x}\right)$. Assume that $a_{0}$ is an extremal vertex. Let $d_{c_{0}}$ denote the direction from $a_{0}$ towards every other essential vertex of $T$. Further assume that $x_{d_{c_{0}}}=n-2+\epsilon_{c_{0}}\left(d_{c_{0}}\right)$, where $\epsilon_{c_{0}}\left(d_{c_{0}}\right)$ is the cup constant associated to $c_{0}$ in direction $d_{c_{0}}$. We call a critical cell of this form extremal. Under these assumptions, we claim that:

Lemma 6.15 (Motivation for more strands). Let $c_{0}=\left(a_{0}, d_{0}, \vec{x}\right)$ be extremal. Then

$$
H^{*}\left(B_{n-2}(T-\{a\})\right) \cong H^{*}\left(B_{n} T\right) \cup c_{0}^{*} .
$$

We will prove this lemma momentarily. Assuming Lemma 6.15 holds, we should be able to reconstruct $T$ by induction: reconstruct $T-\{a\}$ from $B_{n-2}(T-\{a\})$, and then simply 'reattach' $a$ to $T$.

Of course, in general, when we are given a tree braid group we are not given any information about a Morse $T$-embedding or a classification of critical cells. The heart of answering Conjecture 6.14 is in finding cohomology classes which behave like $c_{0}^{*}$ in the sense of Lemma 6.15.

Proof of Lemma 6.15. We wish to construct the isomorphism on cohomology. To do so, we define functions on several types of objects to build up to the desired map: on $b$-vectors for essential vertices $b \neq a$; on reduced 1-cells; on $k$-forms; and finally on cohomology classes.

For any essential vertex $b$ of $T$ which is not $a$, define a function $a t_{b}$ on $b$-vectors, which takes a $b$-vector and adds two (hence the name $a t$ ) to the $\left(d_{b}\right)^{\text {th }}$ coordinate, where $d_{b}$ is the direction from $b$ to $a$. By definition, $a t_{b}$ is injective. Note that $a t_{b}$ is defined regardless of the length of the $b$-vectors.

Now define a function at on reduced 1-cells: at $(b, f, \vec{y}):=\left(b, f, a t_{b}(\vec{y})\right)$. This takes a reduced 1-cell and adds two (again, hence the name) strands in the direction towards $a$. We must require that $b \neq a$. As with $a t_{b}$, the map $a t$ is injective. Note $a t$ is defined regardless of the length of $\vec{y}$. For a reduced $k$-cell $s$, by Theorem 3.5 
there exists a unique collection $\left\{\left[c_{1}\right], \ldots,\left[c_{k}\right]\right\}$ of equivalence classes of 1-cells such that $[s]$ is the least upper bound of $\left\{\left[c_{1}\right], \ldots,\left[c_{k}\right]\right\}$. Define at $(s)$ to be the reduced representative of the least upper bound of $\left\{\left[a t\left(c_{1}\right)\right], \ldots,\left[a t\left(c_{k}\right)\right]\right\}$. We must require that no $c_{i}$ lies over $a$. This corresponds to adding two strands to $s$ in the direction towards $a$ from the essential vertex $b$ closest to $a$ over which one of the $c_{i}$ lies. Think of $a t$ as placing two strands at the vertex $a$ in $T$. Again, as the collection $\left\{\left[c_{1}\right], \ldots,\left[c_{k}\right]\right\}$ is unique, at is injective.

We generalize the map at to $k$-forms, as follows. For a basic $k$-form $\omega=$ $f(b, \vec{y}) d c_{1} \wedge \cdots \wedge d c_{k}$, define $a t(\omega)$ to be

$$
f\left(b, a t_{b}(\vec{y})\right) d\left(a t\left(c_{1}\right)\right) \wedge \cdots \wedge d\left(a t\left(c_{k}\right)\right) .
$$

Here we require that $b \neq a$ and that each $c_{i}$ does not lie over $a$. Again, $a t$ is injective and defined regardless of the number of strands.

We claim that the desired isomorphism on cohomology is the map $A T$, where $A T: H^{*}\left(B_{n-2}(T-\{a\})\right) \rightarrow H^{*}\left(B_{n} T\right) \cup c_{0}^{*}$ is given by

$$
[\omega] \mapsto\left[a t(\omega) \wedge d c_{0}\right],
$$

where $\omega$ is a cochain for $n-2$ strands on the tree $T-\{a\}$, thought of as a cochain for $n-2$ strands on the tree $T$. We prove that $A T$ is well defined with a well-defined inverse map $A T^{-1}$, where

$$
A T^{-1}\left(\left[\omega \wedge d c_{0}\right]\right):=\left[a t^{-1}(\omega)\right]
$$

if $a t^{-1}(\omega)$ is defined; otherwise, $A T^{-1}\left(\left[\omega \wedge d c_{0}\right]\right):=[0]$. If $A T$ and $A T^{-1}$ are well defined, then $A T$ is a ring homomorphism, by definition and Proposition 4.6. Then, clearly from the definitions of $A T$ and $A T^{-1}$, they will be indeed inverse homomorphisms whose composition in either order is the identity, giving the desired isomorphism.

First, consider if $A T$ is well defined. Let $\omega=f(b, \vec{y}) d c_{1} \wedge \cdots \wedge d c_{k} \in[0]$ be a necessary $k$-form, and let $c=(b, f, \vec{y})$ denote the necessary 1-cell for $\omega$. By the definition of necessary, $\left\{\left[c_{1}\right], \ldots,\left[c_{k}\right],[c]\right\}$ has an upper bound $[s]$, and $f$ is the unique respectful edge in the reduced representative $s$ of $[s]$. Consider at $(\omega)$. If at $(\omega)$ is necessary, then $a t(\omega)$ is cohomologous to 0 , and $\left[a t(\omega) \wedge d c_{0}\right]=[0]$. Consider if $a t(\omega)$ is not necessary. Clearly $f$ is such that $\left(b, f, a_{b}(\vec{y})\right)=a t(c)$ is a reduced 1-cell. Moreover, $\left\{\left[a t\left(c_{1}\right)\right], \ldots,\left[a t\left(c_{k}\right)\right],[a t(c)]\right\}$ has an upper bound $[a t(s)]$, by the definition of at. Thus, if at $(\omega)$ is not necessary, $f$ must be disrespectful in at $(s)$, by the definition of necessary. As $f$ is respectful in $s$ but disrespectful in at $(s)$, it must be that the two strands added to $s$ in at $(s)$ are the strands which make $f$ disrespectful. A generalization of the Upper Bound Lemma, which we leave as an exercise for the sake of brevity, shows that $\left\{\left[a t\left(c_{1}\right)\right], \ldots,\left[a t\left(c_{k}\right)\right],[a t(c)],\left[c_{0}\right]\right\}$ has an upper bound $\left[s^{\prime}\right]$, and that moreover $f$ is the unique respectful edge in the reduced representative $s^{\prime}$ of $\left[s^{\prime}\right]$. Thus, at $(c)$ is necessary for the necessary $(k+1)$-form $a t(\omega) \wedge d c_{0}$. Thus, 
regardless of whether $\operatorname{at}(\omega)$ is necessary or not, $\left[\operatorname{at}(\omega) \wedge d c_{0}\right]=[0]$. This shows $A T$ is well defined.

Now, consider if $A T^{-1}$ is well defined. Let $\omega=f(b, \vec{y}) d c_{1}^{\prime} \wedge \cdots \wedge d c_{k}^{\prime}$ be a $k$ form such that $\omega \wedge d c_{0} \in[0]$ is necessary, and let $c^{\prime}=(b, f, \vec{y})$ denote the necessary 1-cell for $\omega \wedge d c_{0}$. By the definition of necessary, $\left\{\left[c_{1}^{\prime}\right], \ldots,\left[c_{k}^{\prime}\right],\left[c_{0}\right],\left[c^{\prime}\right]\right\}$ has an upper bound $\left[s^{\prime}\right]$, and $f$ is the unique respectful edge in the reduced representative $s^{\prime}$ of $\left[s^{\prime}\right]$. Either $a t^{-1}(\omega)$ is defined or not.

If $a t^{-1}(\omega)$ is defined and $a t^{-1}(\omega)$ is necessary, then $a t^{-1}(\omega)$ is cohomologous to 0 , and there is nothing to prove. Consider if $a t^{-1}(\omega)$ is defined but not necessary. Clearly $f$ is such that $\left(b, f, a t_{b}^{-1}(\vec{y})\right)=a t^{-1}\left(c^{\prime}\right)$ is a reduced 1-cell. Moreover, the set of preimages $\left\{\left[a t^{-1}\left(c_{1}\right)\right], \ldots,\left[a t^{-1}\left(c_{k}\right)\right],\left[a t^{-1}(c)\right]\right\}$ has an upper bound $\left[a t^{-1}(s)\right]$, by the definition of at. Thus, if $a t^{-1}(\omega)$ is not necessary, $f$ must be disrespectful in $a t^{-1}(s)$, by the definition of necessary. But if $f$ were disrespectful in $a t^{-1}(s)$, then $f$ would be disrespectful in at $(s)$, a contradiction. Thus, whether or not $a t^{-1}(\omega)$ is necessary, if $a t^{-1}(\omega)$ is defined then $A T^{-1}(\omega)$ is well defined.

If $a t^{-1}(\omega)$ is not defined, then by definition either $a t_{b}^{-1}(\vec{y})$ is not defined or $a t^{-1}\left(c_{i}^{\prime}\right)$ is not defined for some $i \in\{1, \ldots, k\}$. In the former case, let $c^{\prime \prime}:=c^{\prime}$ and in the latter case let $c^{\prime \prime}:=c_{i}^{\prime}$. In either case, express $c^{\prime \prime}$ as $\left(a^{\prime \prime}, d^{\prime \prime}, \vec{z}\right)$, and let $d_{0}^{\prime \prime}$ denote the direction from $a^{\prime \prime}$ to $a_{0}$. Note $\left[c^{\prime \prime}\right]$ and $\left[c_{0}\right]$ have a least upper bound $\left[s^{\prime \prime}\right]$ whose reduced representative $s^{\prime \prime}$ is $\sim$-equivalent to a face of $s$, since $c^{\prime \prime}$ and $c_{0}$ are both $\sim$-equivalent to faces of $s$. As $a t^{-1}\left(c^{\prime \prime}\right)$ is not defined but $\left[s^{\prime \prime}\right]$ is, by the Upper Bound Lemma it follows that the edge $e_{0}$ of $c_{0}$ is respectful in $s^{\prime \prime}$. But then $e_{0}$ is respectful in $s$. This contradicts the assumption that $\omega \wedge d c_{0}$ was necessary. Thus, $a t^{-1}(\omega)$ must be defined and cohomologous to 0 . This proves that $A T^{-1}$ is well defined as a homomorphism.

This finishes the proof.

To conclude this section, we state a theorem which clarifies the behavior of free tree braid groups - that is, tree braid groups on radial trees or on fewer than 4 strands:

Theorem 6.16 (The free case). Let $G$ be a free tree braid group. If the number of strands $n$ for which $G=B_{n} T$ for some tree $T$ is known and $n \geq 4$, $T$ may be reconstructed up to homeomorphism. Otherwise, $T$ may not be uniquely determined up to homeomorphism.

Proof. If $n<4$, then not much can be said about $T$. If $n \geq 4$, then $T$ is radial. If $n$ is known, Theorem 3.4 gives an explicit equation to solve for the degree of the unique essential vertex. The solution is unique since all of the $Y_{n}$ functions are monotone increasing. If $n$ is not known, then it is possible for free groups of ranks given by $r=Y_{n}(x)$ and $r^{\prime}=Y_{n^{\prime}}\left(x^{\prime}\right)$ to be such that $r=r^{\prime}, n \neq n^{\prime}$, and $x \neq x^{\prime}$. For instance: for $n=4, x=4, n^{\prime}=3$, and $x^{\prime}=5$, then $r=r^{\prime}=26$; for $n=5, x=5, n^{\prime}=4$, and $x^{\prime}=6$, then $r=r=155$. In general, for $x^{\prime}=n+1$ and $n^{\prime}=x-1, r=r^{\prime}$. We note without proof, though, that empirically it appears this is the only situation in which $r=r^{\prime}$. 


\section{The isomorphism problem and generalizations}

Theorems 6.9 and 6.16 allow us to solve the isomorphism problem for tree braid groups when we can reconstruct the defining trees by enumeration:

Theorem 7.1 (The Isomorphism Problem). Let $G$ and $G^{\prime}$ be two groups be given by finite presentations, and assume that $G \cong B_{n} T$ and $G^{\prime} \cong B_{n} T^{\prime}$ for some positive integer $n$ and finite trees $T$ and $T^{\prime}$. If either:

- $n=4$ or 5 or

- at least one of $G$ or $G^{\prime}$ is free,

then there exists an algorithm which decides whether $G$ and $G^{\prime}$ are isomorphic. The trees $T$ and $T^{\prime}$ need not be specified. If one of $T$ and $T^{\prime}$ has at least 3 essential vertices, then $n$ need not be specified.

Proof. If both $B_{n} T$ and $B_{n} T^{\prime}$ are free, then the problem reduces to the isomorphism problem for free groups, which has a solution. If exactly one of $B_{n} T$ or $B_{n} T^{\prime}$ is free, then the groups cannot be isomorphic. If both of $B_{n} T$ and $B_{n} T^{\prime}$ are not free, then we reconstruct the trees $T$ and $T^{\prime}$ (and the value $n$ when one of $T$ or $T^{\prime}$ has at least 3 essential vertices) from the corresponding groups, which can be done, according to Theorem 6.9. We need to show that we can reconstruct the trees algorithmically. We begin by extrapolating $T$ from $B_{n} T$.

Let the degree of a tree denote the sum of degrees of all essential vertices of the tree. Note that the number of trees up to homeomorphism of any given degree is finite. Let the length of a presentation for a group denote the sum of lengths of the defining relators in the presentation plus the number of generators. Note that the number of distinct presentations for a group of any given length is finite. For any given homomorphism between two groups and any given presentations of both, let the length of the homomorphism with respect to the presentations be the sum of the lengths of the images of the generators of the first group under the homomorphism. Note that, for fixed groups and presentations, the number of homomorphisms of a given length is finite.

Enumerate all trees up to homeomorphism in some order from lesser degree to greater. For a given tree and a given $n$, enumerate all (reduced) presentations of the corresponding $n$ strand tree braid groups in some order from lesser length to greater. For a given tree, a given $n$, and a given presentation of the corresponding $n$ strand tree braid group, enumerate all homomorphisms to $B_{n} T$ in some order from lesser length to greater. Finally, enumerate all homomorphisms from $n$ strand tree braid groups to $B_{n} T$ by diagonalization.

As we know that $B_{n} T$ is a tree braid group, eventually in this enumeration there will be an isomorphism. Define an algorithm to extrapolate the tree $T$ (up to homeomorphism) and the value $n$ from the group $B_{n} T$ by running through the above enumeration and finding the first isomorphism, and outputting the corresponding tree $T$ and 
value $n$. Similarly, define an algorithm to extract the tree $T^{\prime}$ (up to homeomorphism) from $B_{n} T^{\prime}$ (which can be shortened, since we now know $n$ ).

By Theorems 6.9 and 6.16, the trees $T$ and $T^{\prime}$ are uniquely determined up to isomorphism for the cases of the theorem. Note Corollary 6.12 implies that $n$ is uniquely determined up to isomorphism if either $T$ or $T^{\prime}$ contain at least 3 essential vertices.

Now, the isomorphism problem for $n$ strand tree braid groups has been reduced to solving the homeomorphism problem for trees. But there exists an algorithm for solving this problem, so we are done.

\section{Right-angled Artin groups}

Let $\Delta$ be a finite graph. For the purposes of this paper, we will always assume $\Delta$ is simple: it contains no nontrivial embedded edge loops having less than 3 edges. The right-angled Artin group $G(\Delta)$ associated to $\Delta$ is defined as follows. The group $G(\Delta)$ is generated by the vertices of $\Delta$, and the only relations are that two generators commute if and only if the corresponding vertices are connected by an edge. For more information on right-angled Artin groups, see [5].

The purpose of this section is to state analogues of the main theorems of this paper, but for right-angled Artin groups. Graph braid groups are closely related to right-angled Artin groups, so it is not surprising that similar theorems hold for each. The differences between the two classes of groups is highlighted, however, by the comparative difficulty of proofs for graph braid groups.

For a finite simplicial graph $\Delta$, the flagification of $\Delta$, denoted $\bar{\Delta}$, is the simplicial complex whose vertices are the vertices of $\Delta$, and for which $k$ vertices span a $k$ simplex in $\bar{\Delta}$ if and only if the $k$ vertices span a complete subgraph of $\Delta$. We say $\Delta$ is flag if $\Delta=\bar{\Delta}$. The analogue of Theorem 5.2 is already known, and was proven by Charney and Davis:

Theorem 8.1 (RAAG cohomology; [6]). Let $\Delta$ be a finite simple graph. Then $H^{*}(G(\Gamma)) \cong \Lambda(\bar{\Delta})$.

We remark that in [16] it was proven that a tree braid group $B_{n} T$ is right-angled Artin if and only if $n \leq 3$ or $T$ is linear. The method of proof essentially involved proving, in the non-right-angled Artin cases, that even if the cohomology ring of a tree braid group is an exterior face algebra, it cannot be an exterior face algebra over a flag simplicial complex.

There are two analogues of Theorem 6.9. One is an important rigidity result and appears in the literature, due to Droms. The other does not yet appear in the literature, but follows from Droms's theorem and Gubeladze's theorem:

Theorem 8.2 (RAAG defining graph rigidity; [10]). Let $\Delta$ and $\Delta^{\prime}$ be finite simple graphs. Then $G(\Delta) \cong G\left(\Delta^{\prime}\right)$ if and only if $\Delta \cong \Delta^{\prime}$. 
Theorem 8.3 (RAAG cohomology rigidity). There is a setwise bijection between right-angled Artin groups up to isomorphism and their cohomology rings up to isomorphism.

Proof. By Theorem 8.1, cohomology rings of right-angled Artin groups are always exterior face algebras over flag simplicial complexes. Moreover, every flag simplicial complex corresponds to the cohomology ring of some right-angled Artin group, as there is a bijective correspondence between finite simplicial graphs and flag simplicial complexes. To get from graphs to simplicial complexes, add a $k$-simplex whenever its 1-skeleton is present; to get from flag simplicial complexes to graphs, take the 1-skeleton. Gubeladze's theorem (Theorem 2.3) shows that there is thus a bijective correspondence between cohomology rings of right-angled Artin groups and flag simplicial complexes. As there are bijections between right-angled Artin groups and finite simplicial graphs, finite simplicial graphs and finite flag simplicial complexes, and finite flag simplicial complexes and cohomology rings of right-angled Artin groups, this proves the theorem.

We end with the analogue of Theorem 7.1 for right-angled Artin groups. Theorem 8.2 suggests that the isomorphism for right-angled Artin groups may be solved by reconstructing $\Delta$ given $G(\Delta)$. One simply needs an algorithm for the reconstruction.

Theorem 8.4 (RAAG isomorphism problem). Let $G$ and $G^{\prime}$ be two groups be given by finite presentations, and assume that $G \cong G(\Delta)$ and $G^{\prime} \cong G\left(\Delta^{\prime}\right)$ for some finite simple graphs $\Delta$ and $\Delta^{\prime}$. Then there exists an algorithm which decides whether $G$ and $G^{\prime}$ are isomorphic. The graphs $\Delta$ and $\Delta^{\prime}$ need not be specified.

The proof of this theorem is similar to the proof of Theorem 7.1, and is a standard diagonalization argument. The theorem follows directly from Theorem 8.2, and is, more or less, generally known.

Proof. Let the degree of a graph denote the sum of degrees of all essential vertices. Note that the number of graphs up to homeomorphism of any given degree is finite. Let the length of a presentation for a group denote the sum of lengths of the defining relators in the presentation plus the number of generators. Note that the number of distinct presentations for a group of any given length is finite. For any given homomorphism between two groups and any given presentations of both, let the length of the homomorphism with respect to the presentations be the sum of the lengths of the images of the generators of the first group under the homomorphism. Note that, for fixed groups and presentations, the number of homomorphisms of a given length is finite.

Enumerate all graphs in some order from lesser degree to greater. For a given graph, enumerate all (reduced) presentations of the corresponding right-angled Artin group in some order from lesser length to greater. For a given graph and a given 
presentation of the corresponding right-angled Artin group, enumerate all homomorphisms to $G$ in some order from lesser length to greater. Finally, enumerate all homomorphisms from right-angled Artin groups to $G$ by diagonalization.

As we know that $G$ is a right-angled Artin group, eventually in this enumeration there will be an isomorphism. Define an algorithm to extrapolate the graph $\Delta$ from $G$. Similarly, extrapolate $\Delta^{\prime}$ from $G^{\prime}$. By 8.2, $\Delta$ and $\Delta^{\prime}$ are uniquely determined. Thus, the isomorphism problem for right-angled Artin groups reduces to the isomorphism problem for graphs.

\section{References}

[1] A. Abrams, Configuration spaces of braid groups of graphs. Ph.D. thesis, University of California, Berkeley, 2000.

[2] S. I. Adyan, Algorithmic unsolvability of problems of recognition of certain properties of groups (Russian). Dokl. Akad. Nauk SSSR (N.S.) 103 (1955), 533-535. Zbl 0065.00901 MR 0081851

[3] M. R. Bridson and A. Haefliger, Metric spaces of non-positive curvature. Grundlehren Math. Wiss. 319, Springer-Verlag, Berlin 1999. Zbl 0988.53001 MR 1744486

[4] I. Bumagin, O. Kharlampovich, and A. Miasnikov, The isomorphism problem for finitely generated fully residually free groups. J. Pure Appl. Algebra 208 (2007), 961-977. Zbl 1121.20026 MR 2283438

[5] R. Charney, An introduction to right-angled Artin groups. Geom. Dedicata 125 (2007), 141-158. Zbl 1152.20031 MR 2322545

[6] R. Charney and M. W. Davis, Finite $K(\pi, 1)$ s for Artin groups. In Prospects in topology (Princeton, NJ, 1994), Ann. of Math. Stud. 138, Princeton University Press, Princeton, NJ, 1995, 110-124. Zbl 0930.55006 MR 1368655

[7] F. Connolly and M. Doig, Braid groups and right-angled Artin groups. Preprint 2004. arXiv:math/0411368v1

[8] J. Crisp and B. Wiest, Embeddings of graph braid and surface groups in right-angled Artin groups and braid groups. Algebr. Geom. Topol. 4 (2004), 439-472. Zbl 1057.20028 MR 2077673

[9] M. Dehn, Papers on group theory and topology. Springer-Verlag, New York 1987. MR 0881797

[10] C. Droms, Subgroups of graph groups. J. Algebra 110 (1987), 519-522. Zbl 0625.20026 MR 910401

[11] M. Farber, Topological complexity of motion planning. Discrete Comput. Geom. 29 (2003), 211-221. Zbl 1038.68130 MR 1957228

[12] M. Farber, Instabilities of robot motion. Topology Appl. 140 (2004), 245-266. Zbl 1106.68107 MR 2074919

[13] D. Farley, Homology of tree braid groups. In Topological and asymptotic aspects of group theory, Contemp. Math. 394, Amer. Math. Soc., Providence, RI, 2006, 101-112. Zbl 1102.20028 MR 2216709 
[14] D. Farley, Presentations for the cohomology rings of tree braid groups. In Topology and robotics, Contemp. Math. 438, Amer. Math. Soc., Providence, RI, 2007, 145-172. Zbl 1143.57302 MR 2359035

[15] D. Farley and L. Sabalka, Discrete Morse theory and graph braid groups. Algebr. Geom. Topol. 5 (2005), 1075-1109. Zbl 1134.20050 MR 2171804

[16] D. Farley and L. Sabalka, On the cohomology rings of tree braid groups. J. Pure Appl. Algebra 212 (2008), 53-71. Zbl 1137.20027 MR 2355034

[17] R. Ghrist, Configuration spaces and braid groups on graphs in robotics. In Knots, braids, and mapping class groups-papers dedicated to Joan S. Birman (New York, 1998), AMS/IP Stud. Adv. Math. 24, Amer. Math. Soc., Providence, RI, 2001, 29-40. Zbl 1010.55012 MR 1873106

[18] R. Ghrist and V. Peterson, The geometry and topology of reconfiguration. Adv. in Appl. Math. 38 (2007), 302-323. Zbl 1124.68109 MR 2301699

[19] F. Grunewald and D. Segal, Some general algorithms. I: Arithmetic groups. Ann. of Math. (2) 112 (1980), 531-583. Zbl 0457.20047 MR 595206

[20] J. Gubeladze, The isomorphism problem for commutative monoid rings. J. Pure Appl. Algebra 129 (1998), 35-65. Zbl 0931.20053 MR 1626643

[21] S.-t. Hu, Isotopy invariants of topological spaces. Proc. Roy. Soc. London. Ser. A 255 (1960), 331-366. Zbl 0121.18103 MR 0113226

[22] M. O. Rabin, Recursive unsolvability of group theoretic problems. Ann. of Math. (2) 67 (1958), 172-194. Zbl 0079.24802 MR 0110743

[23] Lucas Sabalka, Braid groups on graphs. Ph.D. thesis, University of Illinois at UrbanaChampaign, 2006. http://math.binghamton.edu/sabalka/SabalkaDissertation.pdf

[24] L. Sabalka, Embedding right-angled Artin groups into graph braid groups. Geom. Dedicata 124 (2007), 191-198. Zbl 1130.20036 MR 2318544

[25] D. Segal, Decidable properties of polycyclic groups. Proc. London Math. Soc. (3) 61 (1990), 497-528. Zbl 0674.20020 MR 1069513

[26] Z. Sela, The isomorphism problem for hyperbolic groups I. Ann. of Math. (2) 141 (1995), 217-283. Zbl 0868.57005 MR 1324134

Received January 22, 2008; revised September 1, 2008

L. Sabalka, Department of Mathematical Sciences, Binghamton University, SUNY,

Binghamton, NY 13902-6000, U.S.A.

E-mail: sabalka@math.binghamton.edu 